\title{
ENCAPSULATION OF PROTEIN MICROFIBER NETWORKS SUPPORTING PANCREATIC ISLETS
}

by

JOSEPH ALLAN MCKINNON STEELE

\author{
A thesis submitted to the Department of Chemical Engineering \\ in conformity with the requirements for \\ the degree of Master of Applied Science
}

\author{
Queen's University \\ Kingston, Ontario, Canada
}

August, 2011

Copyright $@$ Joseph Allan McKinnon Steele, 2011 


\begin{abstract}
A method was developed to produce and incorporate a network of discrete, genipin-crosslinked gelatin microfibers around a pancreatic islet within a barium alginate microcapsule. This technique allows for the encapsulation of a porous fibrous matrix without the geometrical restrictions required for cellular aggregate seeding. Microfibers were produced from a novel vortex-drawn extrusion system with an alginate support matrix. Optimization culminated in a hydrated fiber diameter of $22.3 \pm 0.4 \mu \mathrm{m}$, a $98 \%$ reduction in cross sectional area, while making the process more reliable and less labour intensive. The optimized microfibers were encapsulated at 40 vol\% within $294 \pm 4 \mu \mathrm{m} \mathrm{1.6 \%} \mathrm{barium} \mathrm{alginate} \mathrm{microparticles} \mathrm{by} \mathrm{an} \mathrm{electrostatic-mediated}$ dropwise extrusion system. Pancreatic islets extracted from Sprague Dawley rats were encapsulated within the microparticles, and analyzed over a 21-day preliminary in vitro study. Acridine orange and propidium iodide fluorescent viability staining and light microscopy indicated a significant increase in viability for the fiber-laden particles relative to fiber-free control particles at days 7, 14, and 21. The fiber-laden system also reduced the incidence of disrupted islet cohesion from $31 \%$ to $8 \%$ at day 21 , and showed evidence of islet-fiber adhesion. Preliminary investigations into insulin secretion and metabolic activity showed no significant difference between test and control groups. Further investigation into benefits of islet encapsulation within an extracellular matrix fiber network will be the subject of future studies with this body of work serving as a foundation.

The system developed in this investigation could be developed into a modular scaffold system for tissue engineering beyond the field of islet research.
\end{abstract}




\section{Acknowledgements}

I would like to thank my supervisor Dr. Ron Neufeld for his advice, direction, and willingness to let me blaze my own trail of discovery. I may have wandered from the path and encountered some dead ends along the way, but I got there in the end, and am a better engineer because of it.

To Michael Hrynyk and Katie Reilly, my teammates in the battle royale of the Neufeld lab vs the calibration curve, thank you for sharing the adventure.

Merci beaucoup to Dr. Jean-Pierre Hallé, Dr. Euridice Carmona, Nathalie, Adrianna, Lydia, and François who invited me into their labs, taught me a lot in a very short period of time, and allowed me to "help them with their English".

A special thanks to Dr. Annelise Barron, who inspired the initial trajectory of this investigation.

Thank you to my friends and housemates at Queen's, for ensuring I always had something to celebrate, even if it was just a Labradoodle's birthday. To all my friends from the booming metropolis of Brockville, thank you for always reminding me that there are things in life other than engineering, no matter how many times I forgot.

To my giant family, specifically Mom, Dad, Grandma, and Aunt Marilyn, thank you for all the support over the many years I have been in school (including those to come). I may be moving away, but thanks to Skype I will never be far! 


\section{Table of Contents}

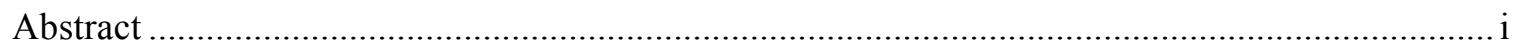

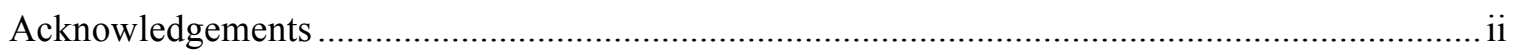

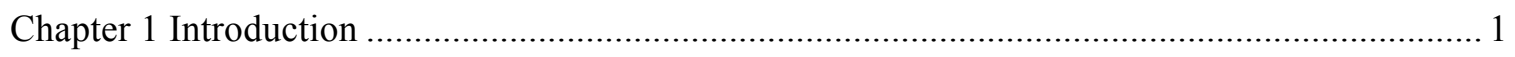

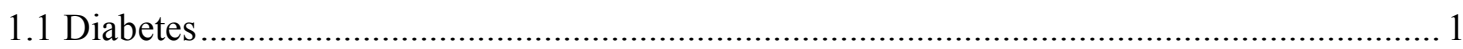

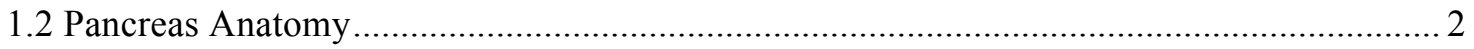

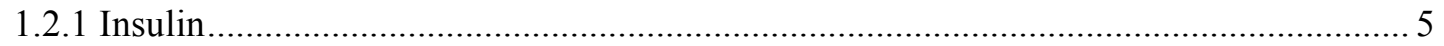

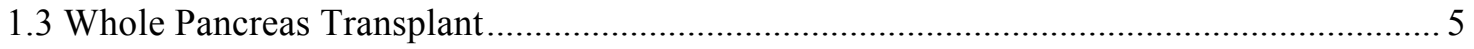

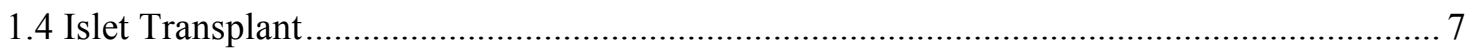

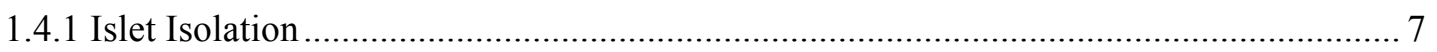

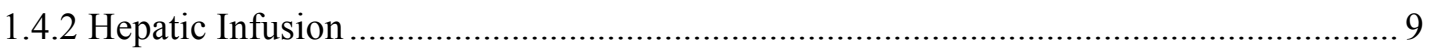

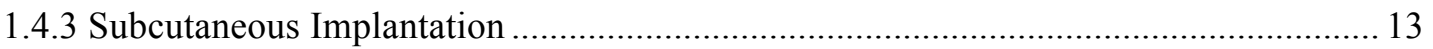

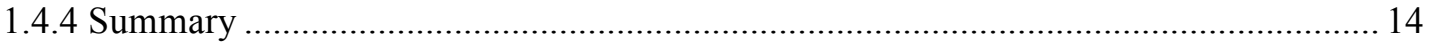

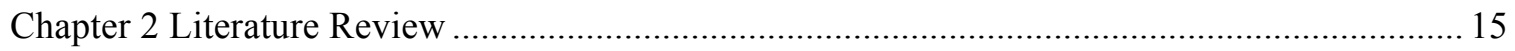

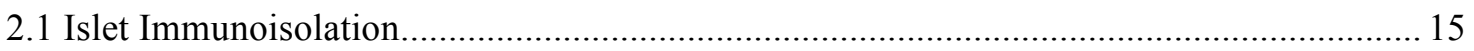

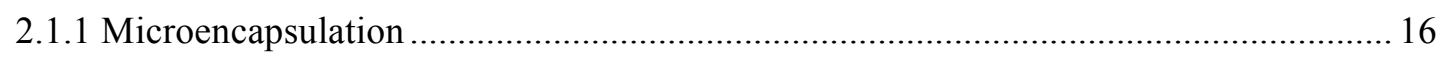

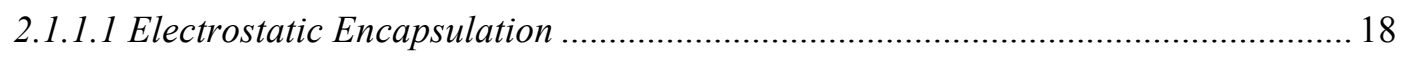

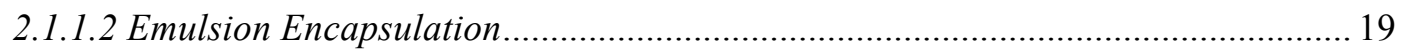

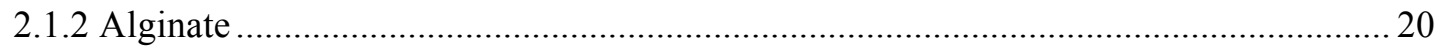

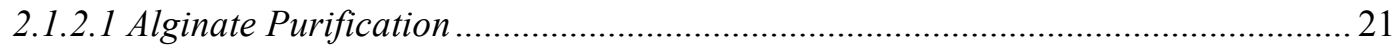

2.1.3 Clinical Trials with Immunoisolated Microcapsules ................................................ 23

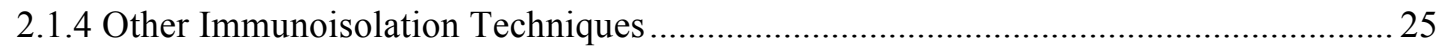

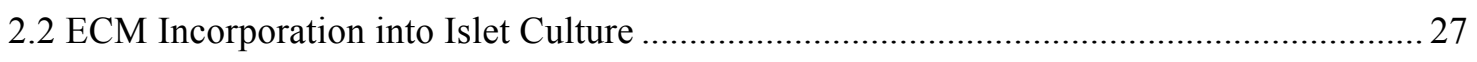

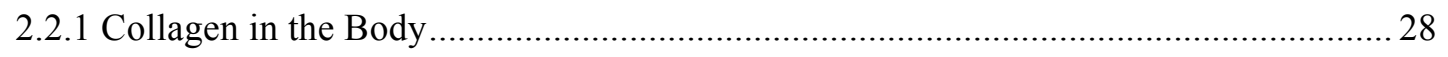

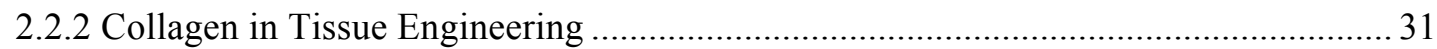




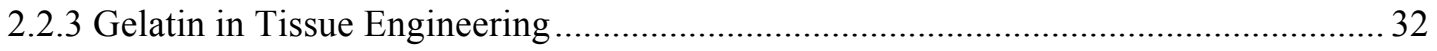

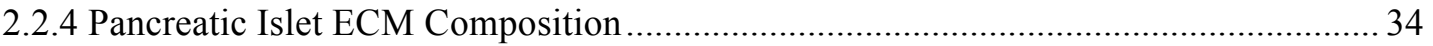

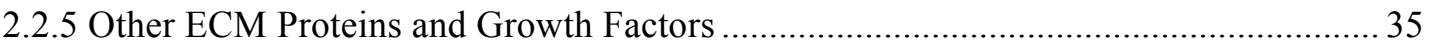

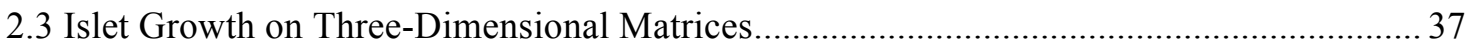

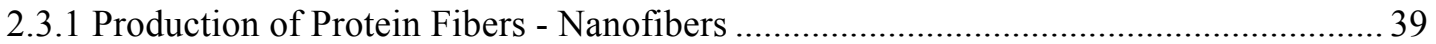

2.3.2 Production of Protein Fibers - Microfibers ................................................................... 42

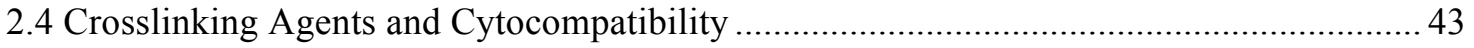

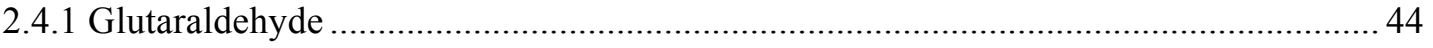

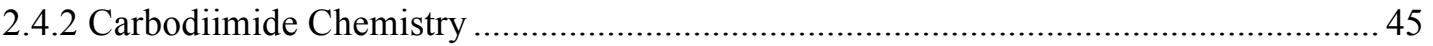

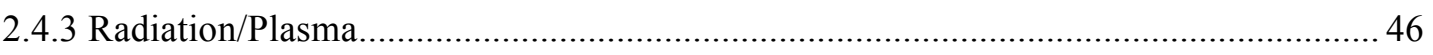

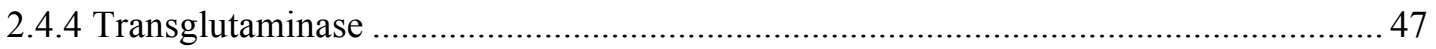

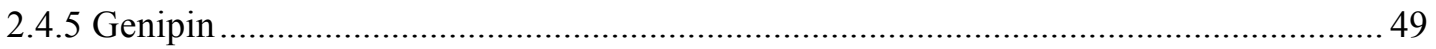

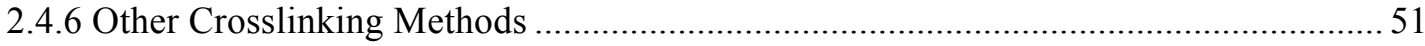

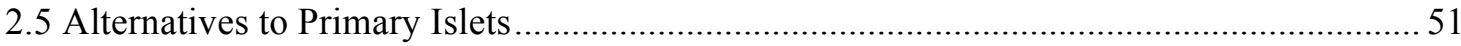

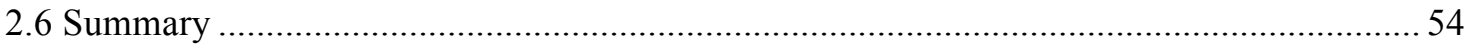

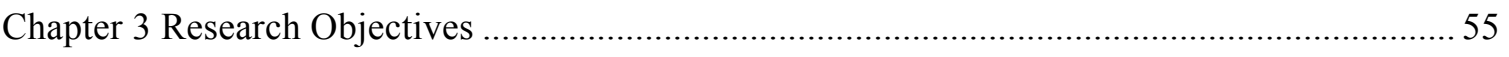

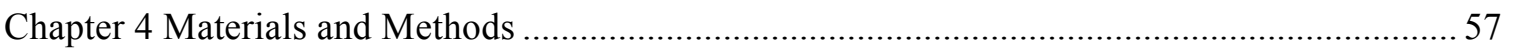

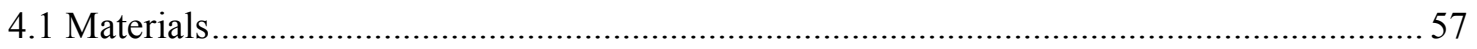

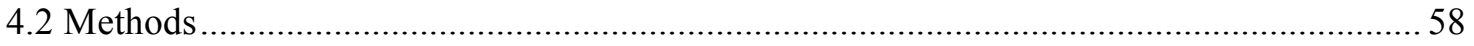

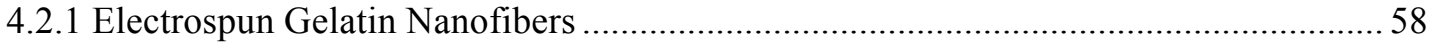

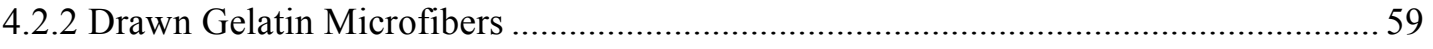

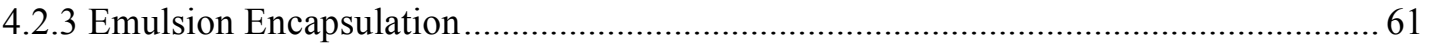

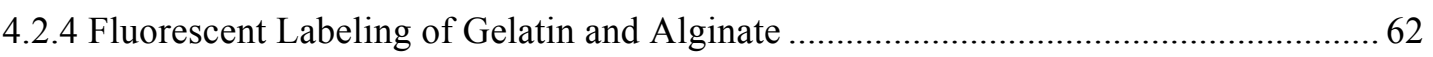

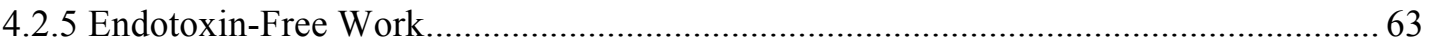

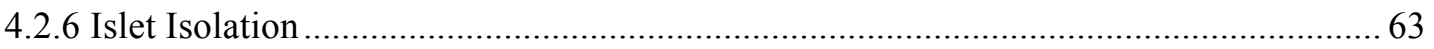




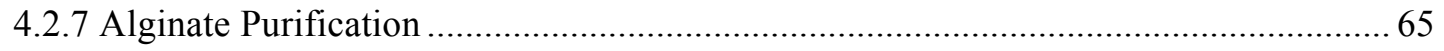

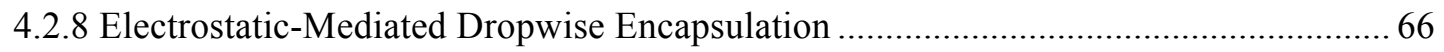

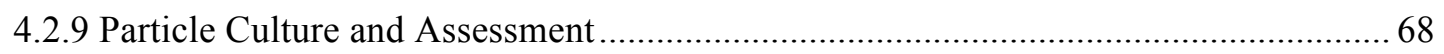

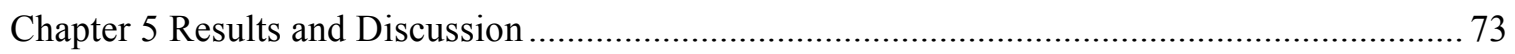

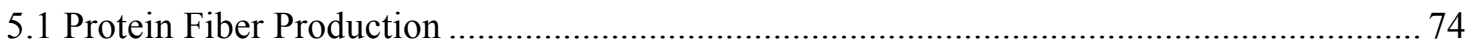

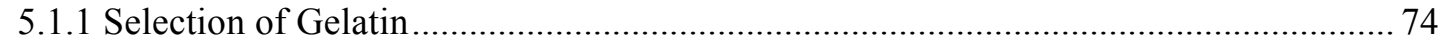

5.1.2 Electrospun Gelatin Nanofiber Investigation............................................................ 74

5.1.3 Drawn Gelatin Microfiber Investigation and Optimization ..................................... 76

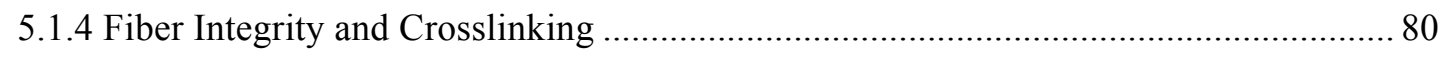

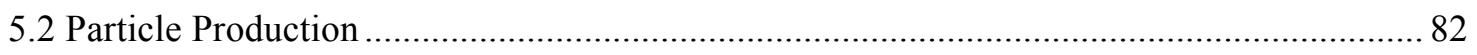

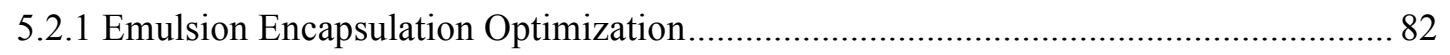

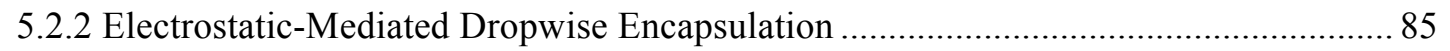

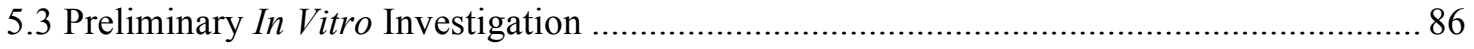

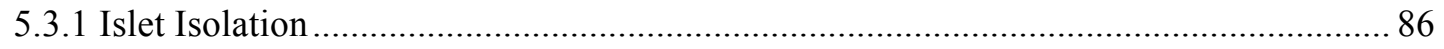

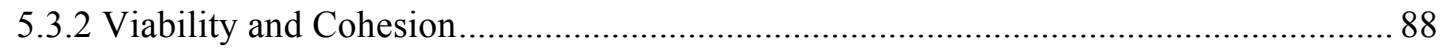

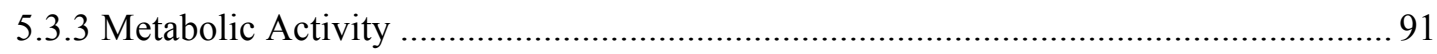

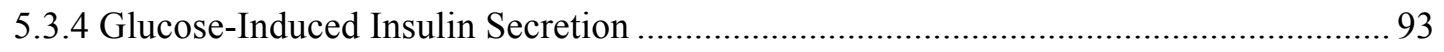

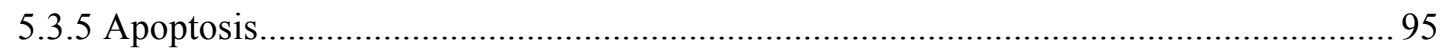

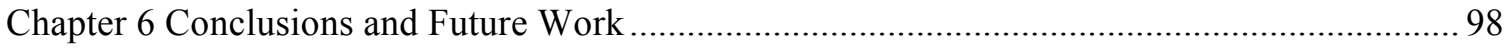

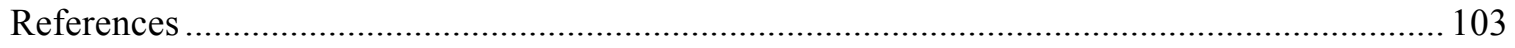

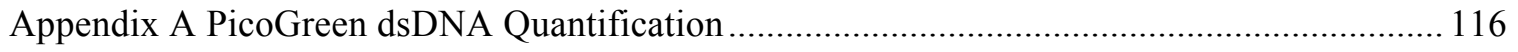

Appendix B Preliminary Protein Membrane Investigation................................................... 119 


\section{List of Figures}

Figure 1. Histology of human islets from Martini et al. (2008). A) Outline of basic islet histology showing arrangement of four main cell types, LM x 400. B) Immunohistochemical staining for glucagon (left) and insulin (right), highlighting the $\alpha$ and $\beta$-cells, LM x 184 . 4

Figure 2. Fluctuations in blood glucose concentrations over a 24-hour period one month before transplantation (A) and after the attainment of insulin independence (B) in a representative patient (Shapiro, 2000). The normoglycemic range falls within the $55-145 \mathrm{mg} / \mathrm{dl}$ zone indicated on the plots

Figure 3. Prevalence of hypoglycemic episodes in patients after their last islet infusion (CITR, 2008)

Figure 4. Chemical structure of sodium alginate, composed of guluronic acid $(\mathrm{G})$ and mannuronic acid (M) monomeric units (Smidsrød, 1990).

Figure 5. Representative images of dithizone-stained islets encapsulated in nonpurified (A) or purified alginate (B) ex vivo after 105 days in the peritoneal cavity of mice (Mallet, 2009). 22

Figure 6. (A,B) Triple helical conformation of collagen molecule $(\mathrm{Gly}-\mathrm{X}-\mathrm{Y})_{\mathrm{n}}$ illustrating the shielding of glycine $(G)$ in the central core of the helix. (C) Chemical structure of glycine (Hulmes, 2008).

Figure 7. Cyclic structure of proline and hydroxyprolines synthesized by proline hydroxylases

(Hulmes, 2008).

Figure 8. Sol-gel transition of gelatin upon cooling (Crescenzi, 2002)

Figure 9. (A) Uncrosslinked gelatin fibers, (B) glutaraldehyde crosslinked (0.5\%), (C) reactive oxygen species crosslinked, (D) glyceraldehyde crosslinked (0.5\%), (E) genipin crosslinked (1\%). Scale bar represents $1 \mu \mathrm{m}$ (Sisson, 2009) 40 
Figure 10. SEM images of uncrosslinked collagen fibers (Control), and fibers crosslinked by EDC/NHS, UV, genipin (GP), and transglutaminase (TG). Scale bar represents $2.5 \mu \mathrm{m}$ (Torres-Giner, 2009).

Figure 11. Crosslinking mechanisms of glutaraldehyde and collagen-bound primary amines

(Sheu, 2001). 45

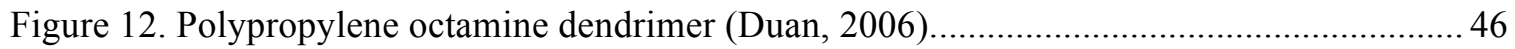

Figure 13. $\varepsilon(\gamma$-glutamyl)lysine linkage formed by transglutaminase (Chen, 2003)..................... 48

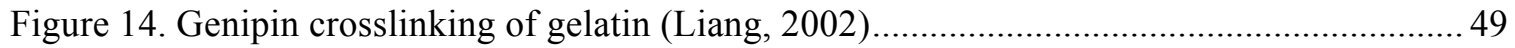

Figure 15. Formation of pancreatic islet-like clusters after UC-PSC (a, b) and BM-MSC (c, d)

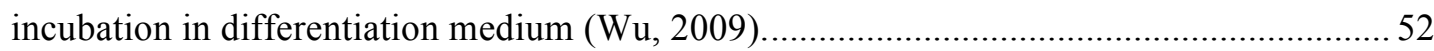

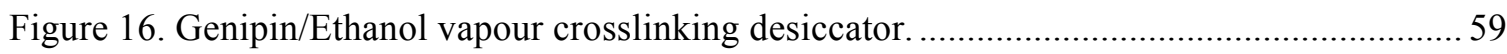

Figure 17. Initial microfiber spinning system with drawing bath and mandrel...........................59

Figure 18. Extrusion of gelatin/alginate solution into a vortex of gelatin and $\mathrm{CaCl}_{2} \ldots \ldots \ldots \ldots \ldots \ldots . . . .60$

Figure 19. Dimensions of impeller and container used in the internally-gelled emulsion system (Poncelet, 1995)

Figure 20. Discontinuous EuroFicoll gradient. Cell mass was loaded in the $1.108 \mathrm{mg} / \mathrm{mL}$ fraction and the purified islet fraction was collected at the $1.096-1.069 \mathrm{~g} / \mathrm{mL}$ boundary following centrifugation.

Figure 21. SEM image of randomly oriented electrospun gelatin nanofibers (scale bar represents $10 \mu \mathrm{m})$

Figure 22. SEM image of genipin-crosslinked gelatin nanofiber sheet (scale bar represents 20

$\mu \mathrm{m})$. .75

Figure 23. Relationship between gelatin concentration within mandrel-drawn $1.5 \%$ alginate microfibers and their properties. Left: Maximum mandrel rotational velocity (draw speed) vii 
and corresponding draw ratios (velocity of collection/velocity of extrusion) as a function of gelatin concentration. Right: Average fiber diameter obtained at the maximum mandrel velocity as a function of gelatin concentration. (Mean $\pm \mathrm{SD}, \mathrm{n}=3$ ).

Figure $24.1 .5 \%$ gelatin $1.5 \%$ alginate mandrel-drawn fibers, with an average hydrated diameter of $108 \pm 1 \mu \mathrm{m}(\mathrm{n}=10)$. .78

Figure 25. Dimensions of rehydrated genipin-crosslinked 1.5\% gelatin microfibers. Left: Hydrated diameter, av $=22.3 \pm 0.4 \mu \mathrm{m}(\mathrm{n}=296) ;$ Right: Length, av $=223 \pm 13 \mu \mathrm{m}(\mathrm{n}=$ 214). 79

Figure 26. SEM images of dehydrated microfibers. Left: Crosslinked fibers are discrete and uniform (scale bar represents $200 \mu \mathrm{m}$ ). Right: Evidence of alignment and fibril formation within the microfibers (scale bar represents $50 \mu \mathrm{m}$ ).

Figure 27. Genipin-gelatin crosslinking and absorbance at $610 \mathrm{~nm}$. Left: Change in the absorbance within the visible spectrum for $10 \%$ gelatin crosslinking with genipin over 120 minutes. Right: Absorbance at $610 \mathrm{~nm}$ as a function of time for three different gelatin concentrations.

Figure 28. Gelatin detected by BCA over a 70 day incubation of fibers in $37^{\circ} \mathrm{C}$ sterile $\mathrm{PBS}$.

$($ Mean $\pm \mathrm{SD}, \mathrm{n}=3)$.

Figure 29. A distribution of fiber densities from different external gelation emulsion systems.

Particles ranged from $600 \mu \mathrm{m}$ to over $3 \mathrm{~mm}$ in diameter......

Figure 30. FITC-labeled islet-like particles (green) encapsulated within a microfiber network

(red) 83

Figure 31. Comparison between traditional and fiber-laden emulsions 84

Figure 32. Bimodal distribution of large, dense particles (left) and small fiber-free particles (right) produced by an 8 minute emulsion (scale bars represent $1000 \mu \mathrm{m}$ ). 84 viii 
Figure 33. Electrostatic-mediated dropwise encapsulation particle size distribution.

Figure 34. Alginate particles containing gelatin networks produced by both techniques, on a common scale. Left: emulsion, Right: electrostatic-mediated dropwise. 86

Figure 35. Left: Size distribution of isolated islets. Right: Image of isolated islets. 87

Figure 36 . AO/PI viability assay classification. 1 - completely red/colourless, 2 - more than 50\% red/colourless, 3 - more than 50\% green, 4 - less than 10\% red/colourless cells, 5 completely green. 88

Figure 37. Viability of rat islets encapsulated within fiber-laden and no-fiber control particles over a period of 21 days. Left: Viability assessment based on the 1-2/3-5 non-viable/viable classifications. Right: Average viability ranking, (mean \pm SE, day 1: $\mathrm{n}>50$, days 7-21: $\mathrm{n}>$ $100),{ }^{*}$ statistically different $(\mathrm{p}<0.01)$

Figure 38. Left: Cohesive islet distribution under AO/PI staining, Center: Disrupted islet distribution with ( $>3$ clusters), Right: Percent of viable islets disrupted as a function of time $(n>100)$.

Figure 39. Islet adhesion to encapsulated gelatin fibers. Left: A single $\beta$-cell adhered to a gelatin fiber, observed in a section stained with H\&E. Right: An islet adhered to a gelatin fiber observed under AO/PI viability staining.

Figure 40. Corrected absorbance for the CellTiter $96^{\circledR}$ Aqueous One Solution Cell Proliferation assay after different culture durations over 21 days. Left: 4 hours, Right: 24 hours. (Mean \pm SE, $n=3)$ 92

Figure 41. Insulin secretion over a 1-hour incubation in $3.3 \mathrm{mM}$ glucose RPMI at days 1, 7, 14, and 21 for no-fiber (NF) and fiber-laden (F) particles divided into their 3.3, 5.5, 11, and 16.5 $\mathrm{mM}$ test groups for comparison with data in Figure 42. (Mean $\pm \mathrm{SE}, \mathrm{n}=3$ ) 94 
Figure 42. Insulin secretion over a 1-hour incubation in $3.3,5.5,11$, or $16.5 \mathrm{mM}$ glucose RPMI immediately following a $3.3 \mathrm{mM}$ glucose incubation (Figure 41) at days 1, 7, 14, and 21 for no-fiber (NF) and fiber-laden (F) particles. (Mean $\pm \mathrm{SE}, \mathrm{n}=3$ ).

Figure 43. Stimulation index for 50 particles cultured under different test concentrations of glucose (Figure 41, Figure 42) at days 1, 7, 14, and 21 for no-fiber (NF) and fiber-laden (F) particles. (Mean $\pm \mathrm{SE}, \mathrm{n}=3$ ) 95

Figure 44. Rat islet sections stained with Hoechst 33258 and observed with a cyan excitation filter. Left: normal/round nuclei, Right: condensed/irregular nuclei. 95

Figure 45. Nuclei in Hoechst-stained sections with a condensed/irregular morphology over 21 days for fiber-laden particles and the fiber-free controls. (Mean $\pm \mathrm{SE}, \mathrm{n}=7$ to 17 ) 96

Figure 46. Particle loss during sectioning and slide preparation. Displaced particle sections (dark grey) visible on a background of low melting point agarose (light grey) and voids (white) initially formed by particles.

Figure 47. Calibration curve for the standard and $10 \mathrm{mM}$ EDTA PicoGreen assays. (Mean $\pm \mathrm{SD}$, $\mathrm{n}=3)$

Figure 48. Double stranded DNA content of the glucose tolerance test samples at day 21 as measured by PicoGreen assay. (Mean $\pm \mathrm{SE}, \mathrm{n}=3$ ).

Figure 49. Swelling of alginate particles with and without transglutaminase-crosslinked gelatin membranes. Left: particles with crosslinked gelatin membranes upper left, particles without membranes bottom right, before chelation; Right: after 40 min in chelating buffer 120

Figure 50. Swollen and ruptured transglutaminase-crosslinked gelatin membranes. 120

Figure 51. Confocal microscopy image of a FITC-gelatin (green) membrane around a RBITCalginate (red) core. 


\section{List of Tables}

Table 1. Summary of human-islet isolation data from human-to-human clinical trials

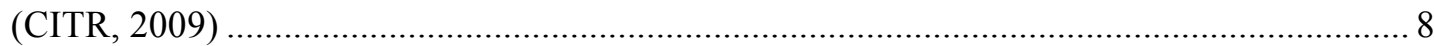

Table 2. Islet infusion clinical trial summary (CITR 2009) .................................................... 9

Table 3. Amino acid composition of gelatin derived from porcine skins $( \pm 0.05 \%)$

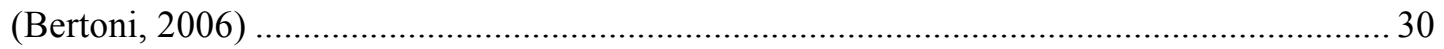

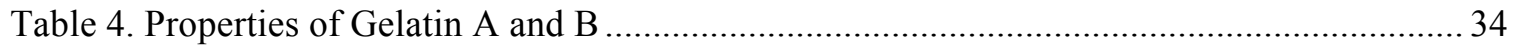

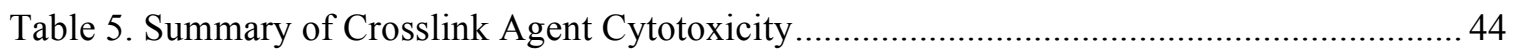




\section{Chapter 1}

\section{Introduction}

\subsection{Diabetes}

Type I diabetes mellitus is an autoimmune disease resulting from the T-lymphocyte-mediated destruction of pancreatic islets (Atkinson, 2005). In healthy pancreatic islets, insulin is produced in response to elevated blood glucose levels in order to maintain a state of normoglycemia. Insulin is distributed systemically through the blood, signaling the liver, adipose, and muscle to absorb glucose and store it as glycogen and triglycerides (Flakoll, 2004). If the insulin response is absent or insufficient, the concentration of glucose in the blood accumulates, a condition termed hyperglycemia. Chronic hyperglycemia results in serious medical complications including atherosclerosis, kidney failure, blindness, peripheral nerve damage, stroke, coronary heart disease, limb amputation and depression (Williams, 2006; CDA, 2011). Obversely, low blood sugar, termed hypoglycemia, is also a serious concern for those with diabetes, as unregulated blood glucose levels are highly labile and can fall to dangerously low levels with little warning (Skarulis, 2004). Hypoglycemic episodes are characterized by a spectrum from discomfort through dizziness to unconsciousness, resulting in serious brain damage and eventually death if glucose levels are not restored (Skarulis, 2004). Before the discovery and wide availability of insulin, diabetes was a fatal disease, with many children dying of the effects of ketoacidosis (Williams, 1894).

Currently, type I diabetes is managed by multiple daily insulin injections, blood sugar monitoring, and carefully controlled diet and exercise. However, these non-continuous monitoring and 
adjustment schemes simply retard the progression of the disease (Kobayashi, 2008). In addition, insulin therapy to reduce hyperglycemic spikes decreases the risk of complications but increases the risk of life-threatening hypoglycemic episodes (DCCT, 1993).

There are currently over 2.7 million Canadians living with diabetes and this number is expected to rise to 4.2 million by 2020 (CDA, 2011). Type I diabetes only accounts for $10 \%$ of patients with diabetes, however it develops much earlier, traditionally during adolescence, resulting in an increased number of chronic complications and a 15 year average reduction in life expectancy (CDA, 2008). The total cost of diabetes to the Canadian health care system and economy in 2010 was approximately $\$ 11.7$ billion. With increasing rates of diabetes and associated costs, that value is expected to reach $\$ 16$ billion by 2020 (CDA, 2011).

The increasing cost of diabetes treatment has promoted continued effort and funding to combat diabetes and the detrimental effects of labile blood sugar.

\subsection{Pancreas Anatomy}

Before discussing the different approaches toward the engineering of a biomimetic endocrine pancreas, a basic understanding of the physiological system is needed. The following is a brief description of pancreas anatomy (Martini, 2008).

The human pancreas is a $20-25 \mathrm{~cm}$ long, $80 \mathrm{~g}$, nodular pink gland that lies within the abdominal cavity between the stomach and the small intestine. Roughly $99 \%$ of the pancreatic volume is composed of exocrine cells that produce digestive enzymes and buffers and excrete them into the duodenum via the pancreatic duct. The remaining $1 \%$ of the volume is occupied by endocrine structures termed pancreatic islets or islets of Langerhans, which are scattered throughout the pancreas and account for roughly two million cells in the average adult human. To serve their 
endocrine function, the islets are heavily vascularized via a capillary network supplied by the pancreaticoduodenal and pancreatic arteries, and drained by the hepatic portal vein, which flows directly into the liver.

The islet structure is composed primarily of four endocrine cell types, as can be seen in Figure 1A (Martini, 2008). $\alpha$-cells produce glucagon, which raises blood glucose by increasing the rate of glycogen breakdown and hepatic glucose release. $\beta$-cells produce insulin, which lowers blood glucose by increasing the rate of glucose uptake by most cells. Composing roughly $85 \%$ of the cells within an islet, $\beta$-cells are the most prevalent (Hallé, 2009). $\delta$-cells produce somatostatin, which inhibits the production and secretion of both glucagon and insulin while slowing the rate of food absorption and enzyme secretion in the digestive tract. F-cells (pp-cells) produce pancreatic polypeptide, which inhibits gallbladder contractions and regulates the production of some pancreatic enzymes. A fifth cell type, not shown in Figure 1A is $\varepsilon$-cells, which produce the hormone ghrelin to stimulate hunger (Hallé, 2009). Only $\alpha$ and $\beta$-cells are sensitive to blood glucose levels, and are therefore the most prominently discussed and observed cells for the purpose of endocrine pancreas engineering (Perez, 2009). The distribution of $\alpha$ and $\beta$-cells within the islet can be identified by immunohistochemical staining for glucagon and insulin, respectively, shown in Figure 1B. 

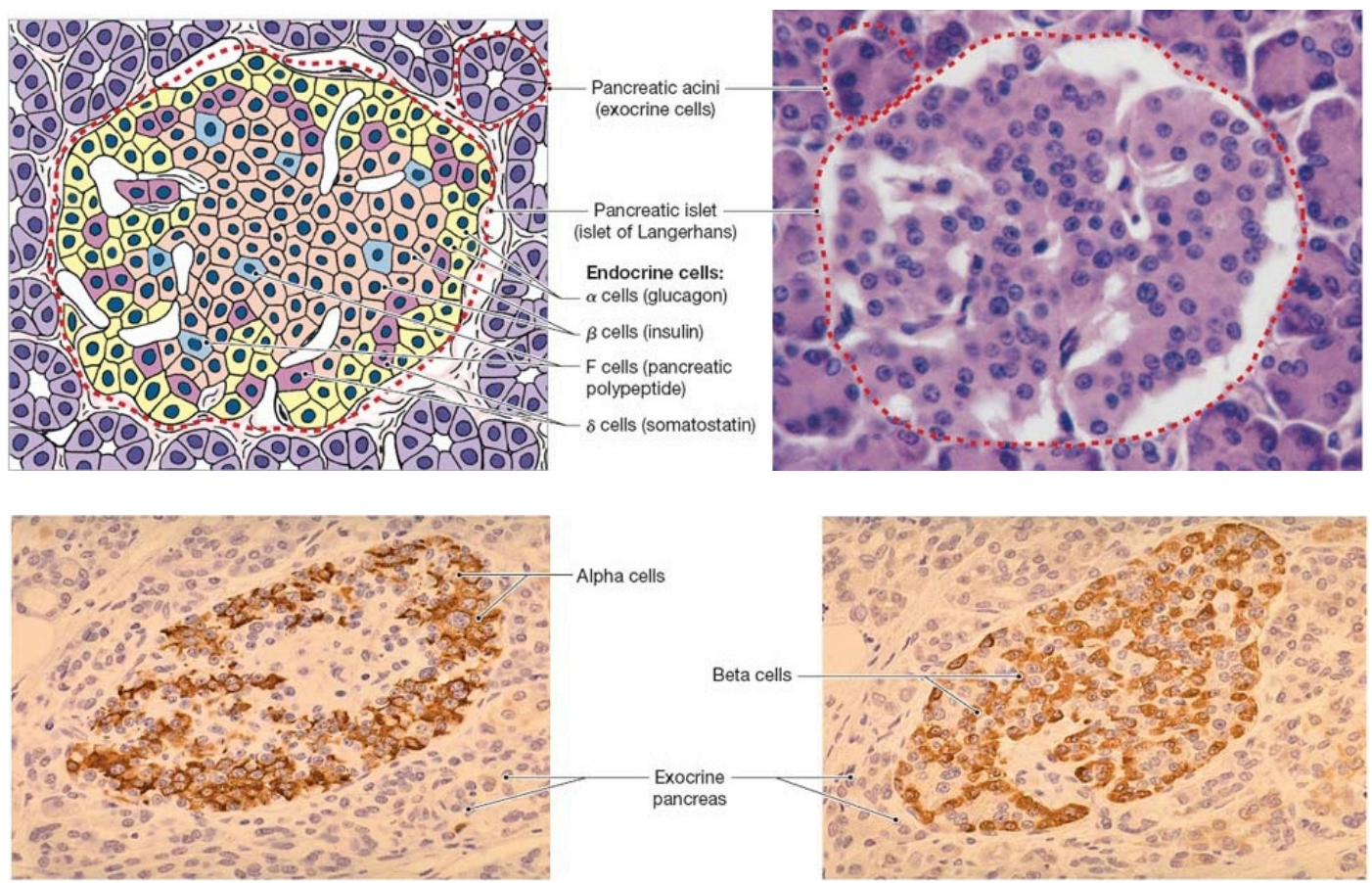

Figure 1. Histology of human islets from Martini et al. (2008). A) Outline of basic islet histology showing arrangement of four main cell types, LM x 400. B) Immunohistochemical staining for glucagon (left) and insulin (right), highlighting the $\alpha$ and $\beta$-cells, LM x 184.

The hormone-laden blood from the pancreas drains to the liver, where approximately half the insulin is consumed (Duckworth, 1998). The pancreas secretes insulin and glucagon in an antisynchronous pulsatile manner with a period of 3-6 minutes and amplitudes of 100-800 pM and 10-120 pM respectively (Hellman, 2009). It is believed that this pulsatile flow with alternating peaks of insulin and glucagon evolved in order to prevent insulin receptor down-regulation within the hepatic tissue, the primary energy storehouse of the body (Hellman, 2009). 


\subsubsection{Insulin}

The insulin precursor proinsulin is synthesized by $\beta$-cells. The $\beta$-cells then cleave proinsulin into the 51 amino acid, $5808 \mathrm{Da}$ insulin molecule which is composed of A and B chains joined by disulphide bonds, and a 31 amino acid C-peptide (Elliott, 2007). C-peptide production is often quantified to measure the rate of insulin production in vivo. This is especially true for xenogenic grafts, as the C-peptide differs between mammalian species, allowing one to ensure that the insulin is being produced by the transplant and not by residual host pancreatic tissue (Brandenburg, 2008). Insulin concentration can be precisely quantified by enzyme-linked immunosorbent assay (ELISA).

\subsection{Whole Pancreas Transplant}

As the pancreatic islets in patients with type I diabetes have been destroyed, any tissue engineering approach will require the replacement of the lost endocrine tissue. The most direct approach is that of whole pancreas transplantation. Xenogenic pancreas transplantation in part or as a minced injectable, was used as early as 1894, almost 30 years before the discovery of insulin, for the temporary relief of the symptoms of diabetes mellitus which was, at that time, inevitably fatal (Williams, 1894). Modern allogenic whole pancreas transplant, first performed in 1966, is still the gold standard endocrine replacement treatment, with over 23,000 transplants reported to the International Pancreas Transplant Registry to date (IPTR, 2009). However, unlike heart, lung, and liver transplants, it is not a life-saving operation, but one to improve quality of life. Benefits to type I diabetics include the elimination of exogenous insulin therapy and the health risks and chronic complications associated with hypo- and hyperglycemic episodes. However, the longterm advantages of glycemic control must be balanced against the severity of the operation, and 
the side-effects of life-long immunosuppression required to prevent alloimmunity and autoimmune reoccurrence (White, 2009).

As $99 \%$ of the cell mass of the pancreas is composed of exocrine cells, the flow of digestive enzymes from the implanted tissue must be managed. The exocrine secretions are drained either to the bladder or the bowel by anastomosis of the pancreatic duct, causing issues including acidosis, thrombosis, dysuria, upper urinary tract infections, haematuria, dysplasia, sepsis, and pancreatitis. Due to the delicate nature of the anastomoses, up to $25 \%$ of the procedures require alterations within 10 years, risking the complete loss of the graft (Sollinger, 1992).

Unfortunately, the number of transplant-quality pancreases is extremely low, as the ideal donor would be brain-dead with a functioning heart, under the age of 50 with a BMI under $30 \mathrm{~kg} / \mathrm{m}^{2}$. However, the pancreases of donors who fall outside the BMI restriction can still be used for islet transplant as the gross fatty infiltration which makes them unsuitable for whole pancreas transplant is eliminated during islet isolation (Stegall, 2007).

With current techniques, the serious nature of the transplant surgery and the side-effects of lifelong immunosuppression are too great to warrant whole pancreas transplant in any circumstance other than severe uremic diabetes where a kidney transplant is also required, or in the case of lifethreatening hypoglycemia and severe secondary complications (White, 2009). Therefore, another approach must be developed to improve glycemic control in the majority of the population afflicted with type-1 diabetes. The first step in the engineering of a cell-based treatment for diabetes is to isolate the cells of interest, discarding the bulk of the pancreas, the superfluous exocrine tissue, and focusing on the islets which compose the endocrine tissue. 


\subsection{Islet Transplant}

Implantation of whole islets initially proposed by Lacy and Kostianovsky (1967) is preferred over whole-pancreas or $\beta$-cell transplant, as islets can be easily isolated, quantified, and implanted, while retaining the complex glucose-modulating multicellular interactions of the endocrine pancreas functional subunit (Hallé, 2009). Dividing the islets further into their constituent cells and implanting purified insulin-producing $\beta$-cells reduces the body's ability to suppress insulin secretion during a hypoglycemic episode. Islet dissociation also eliminates the cell-cell interactions found within the aggregated islet state which are required for glucose responsiveness and have been shown to up-regulate insulin secretion (Bosco, 1997).

The majority of islet implantation sites are located such that the secreted insulin enters circulation via the portal venous system, either directly through the hepatic portal vein or through the venous drainage of the peritoneal cavity. This hepatic drainage replicates the natural arrangement where the hormone-laden blood drains from the pancreas to the hepatic portal vein via the pancreatic, pancreaticoduodenal and splenic veins (Martini, 2008).

\subsubsection{Islet Isolation}

The human islet isolation technique used in the majority of clinical applications, such as the one used in the Edmonton Protocol (Shapiro, 2000), uses pancreases from brain-dead donors which are removed once the aorta is clamped and stored in chilled organ transplantation solution. A commonly used organ transplantation solution is University of Wisconsin solution (ViaSpan $\left.{ }^{\circledR}\right)$, which maintains osmotic pressure while preventing edema, hyperuricemia, and free-radical damage. The pancreatic duct is then infused with chilled Liberase solution, a mixture of collagenases and thermolysin (Roche, 2008), to digest the pancreatic extracellular matrix (ECM). 
Islets are then separated from the ECM by gentle mechanical dissociation and purified via a density gradient of Ficoll-diatrizoic acid in an apheresis system. In place of xenoprotein products, the isolation and purification media contain 25\% human albumin (Shaprio, 2000). In $46 \%$ of clinical applications, islets are used without pretreatment, in the remaining $54 \%$ of cases, islets are cultured for 27 hours on average, allowing the islet population to be checked for contamination while maintain viability and providing time to prepare for transplantation (CITR, 2009).

Table 1 summarizes the islet yields from human-to-human transplants reported to the CITR between 1999 and 2008. The Islet Equivalent (IEQ) is a standard volumetric unit of measure for islet studies, defined as a sphere with a diameter of $150 \mu \mathrm{m}$. As can be seen in

Table 1, the number of islet particles is well approximated by IEQ. It is important to take notice of the number of IEQ isolated from a single adult pancreas, as many techniques use cell quantities far higher than the physiological quantity, requiring multiple donors (Tuch, 2009).

Table 1. Summary of human-islet isolation data from human-to-human clinical trials (CITR, 2009)

\begin{tabular}{llllll}
\hline & N & Mean & SD & Min & Max \\
\hline IEQ post purification $\left(\times 10^{3}\right)$ & 403 & 384 & 156 & 85 & 973 \\
Total cell volume $(\mathrm{mL})$ & 805 & 3.5 & 2.1 & 0.1 & 16.0 \\
Islet particle count $\left(\mathrm{x} 10^{3}\right)$ & 683 & 364 & 158 & 63 & 996 \\
IEQ $\left(\times 10^{3}\right)$ & 721 & 388 & 162 & 54 & 1,122 \\
IEQ $\left(\times 10^{3}\right) / \mathrm{kg}$ donor weight & 700 & 4.62 & 1.92 & 0.72 & 17 \\
Beta cells $\left(\times 10^{6}\right)$ & 266 & 264 & 179 & 4 & 975 \\
\hline
\end{tabular}

Extraction of islets from rats is a very similar process, with expectedly lower yields of approximately 400 IEQ per rat. To ensure maximum islet viability, rats are anesthetized until pancreatic duct cannulation whereupon they are sacrificed before injection of chilled collagenase into the pancreatic duct followed by mechanical excision of the collagenase-swollen tissue. 


\subsubsection{Hepatic Infusion}

Many different implantation techniques have been developed for both human and animal models. The most widely instituted islet transplantation method in human-to-human clinical applications is intraportal hepatic infusion (CITR, 2009).

The first case of human insulin-independence after hepatic infusion of pancreatic islets was reported in 1989 (Scharp, 1990). In their Annual Report for 2009, the Collaborative Islet Transplant Registry reported that between 1999 and 2008, 783 human-to-human islet transplant procedures have been performed on 408 patients at 31 transplant centers throughout North America (CITR, 2009).

Most clinical research groups have found it necessary to use multiple infusions of islets, drawing from multiple donors. The summary of islet infusions between 1999 and 2008 reported to the CITR is presented in Table 2 .

Table 2. Islet infusion clinical trial summary (CITR 2009)

\begin{tabular}{cccccccc}
\hline \multirow{2}{*}{ Infusion } & \multicolumn{3}{c}{ IEQ Infused } & & \multicolumn{3}{c}{ IEQ Infused/kg } \\
\cline { 2 - 4 } \cline { 7 - 9 } & $\mathbf{N}$ & Mean & SD & & N & Mean & SD \\
\hline $\mathbf{1}$ & 334 & 447,000 & 155,000 & & 330 & 6,800 & 2,530 \\
$\mathbf{2}$ & 234 & 415,000 & 164,000 & & 223 & 6,450 & 2,580 \\
$\mathbf{3}$ & 77 & 412,000 & 160,000 & & 74 & 6,500 & 2,790 \\
\hline
\end{tabular}

The most prominent breakthrough in the field of islet transplant to date occurred in 2000 when the Edmonton Protocol by Shapiro et al. (2000) resulted in seven out of seven islet recipients becoming insulin-independent at one year, with steroid-free immunosuppression. Islets were infused into the main portal vein via a catheter which gravity-fed $120 \mathrm{~mL}$ of medium containing more than $4000 \mathrm{IEQ} / \mathrm{kg}, 500 \mathrm{U}$ of heparin, and 20\% human albumin. Insulin therapy was stopped immediately. It was deemed necessary to follow up with a second infusion in each of the seven 
patients on average 29 days after the first procedure. In the most obese individual, a third procedure was also required. In the end, $11,500 \pm 1,600 \mathrm{IEQ} / \mathrm{kg}$ were required to achieve insulin independence, compared to the $4,620 \pm 1,920 \mathrm{IEQ} / \mathrm{kg}$ found in the average donor (Table 1). The average hospitalization was 2.3 days, which was reduced to 24 hours for the final three patients, and no patients experienced acute rejection. The success of the Edmonton Protocol in 2000 is illustrated most vividly in Figure 2 which displays the range of glucose concentrations in a representative patient's bloodstream for a given day before the procedure and after insulinindependence was achieved.

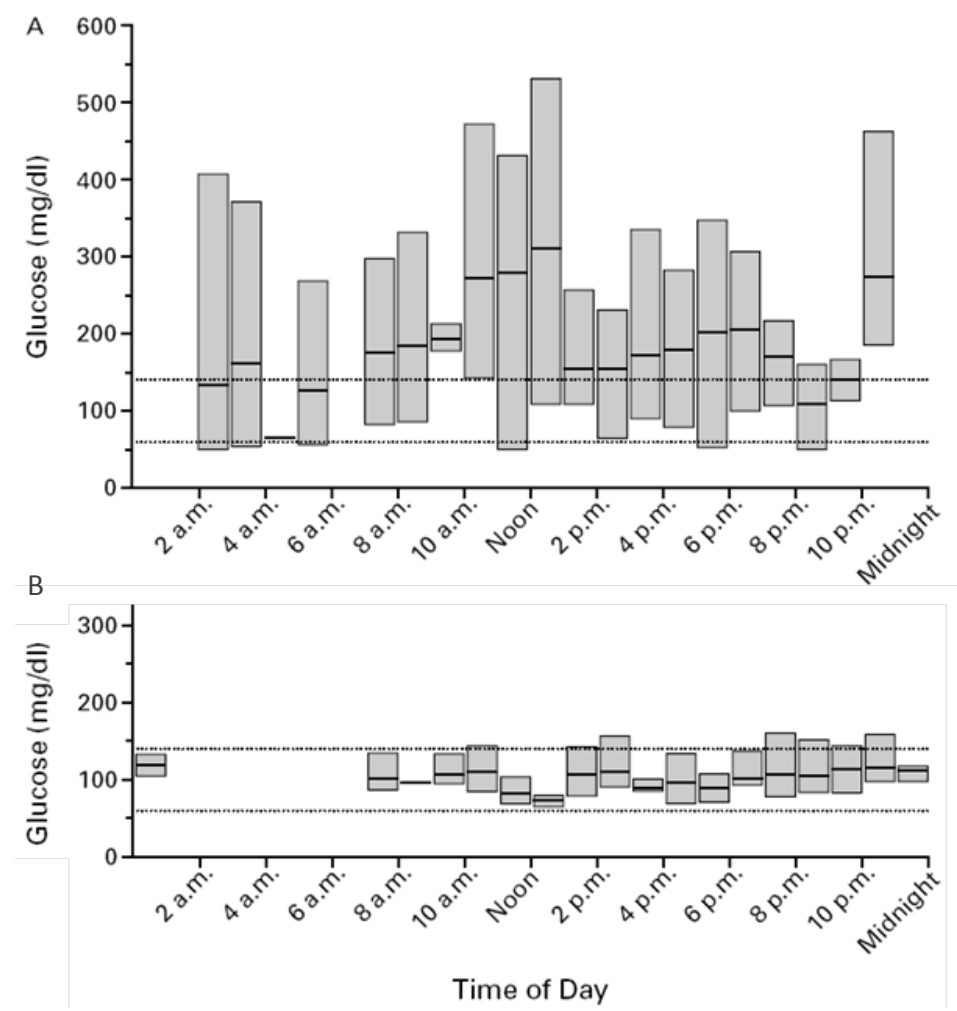

Figure 2. Fluctuations in blood glucose concentrations over a 24-hour period one month before transplantation (A) and after the attainment of insulin independence (B) in a representative patient (Shapiro, 2000). The normoglycemic range falls within the 55-145 $\mathrm{mg} / \mathrm{dl}$ zone indicated on the plots. 
The restoration of normoglycemia with glucocorticoid-free immunosuppression was a sign that the field was maturing into a clinically viable treatment for type I diabetes. However, an international trial of the Edmonton Protocol in 2006 (Shapiro, 2006) was less successful at achieving insulin independence. The study involved 36 patients at 9 sites, only 16 of whom (44\%) achieved insulin independence at one year. Of the remainder, 10 had partial function and 10 had complete graft loss at one year. The unexpectedly poor results were attributed to inexperience. At sites where four or more procedures were performed in the previous two years, 12 of $18(67 \%)$ patients achieved insulin independence at one year, while only 4 of $18(22 \%)$ patients at less experienced sites achieved the same result.

Side effects reported in the Edmonton Protocol's international trial were primarily due to the immunosuppression. The only significant procedure-related issues were with intraperitoneal bleeding (9\%) and partial branch-vein occlusions $(6 \%)$. The side effects of the glucocorticoid-free immunosuppression (daclizumab, sirolimus, and tacrolimus) were mouth ulceration (92\%), anaemia (81\%), leucopenia (75\%), diarrhea $(64 \%)$, headache $(56 \%)$, neutropenia $(53 \%)$, nausea (50\%), vomiting (42\%), acne (39\%), and fatigue (39\%). The immunosuppression therapy was changed from the Edmonton Protocol standard in $25 \%$ of the patients due to side-effects (Shapiro, 2006).

Despite the poorer results, and complications from immunosuppression, the study succeeded in standardizing pancreas selection, islet processing, product-release criteria, recipient selection, and post-transplantation care under a FDA investigational new drug submission, while highlighting the need for less toxic immunosuppressive therapy (Shapiro, 2006).

Of the 279 hepatic islet-transplant patients registered by the CITR between 1999 and 2004, 23\% retained insulin independence at 3 years post first infusion, 29\% maintained detectable levels of 
c-peptide, despite resuming exogenous insulin therapy, $26 \%$ had complete graft failure with no detectable c-peptide levels, and 22\% had unreported outcomes at 3 years (CITR, 2008). Despite low levels of insulin independence, many patients report reduced exogenous insulin demands, and experienced a significant reduction in hypoglycemic episodes. Throughout the first year, the prevalence of severe hypoglycemic events was reduced from $81-85 \%$ pre-infusion to less than 3 $10 \%$, only increasing to $8-35 \%$ at three years post last infusion (Figure 3 ) with hypoglycemic episodes only occurring in patients that had resumed exogenous insulin therapy. Patients with full and partial graft survival experienced increased hypoglycemic awareness, allowing them to better control their insulin regimen (CITR, 2008).

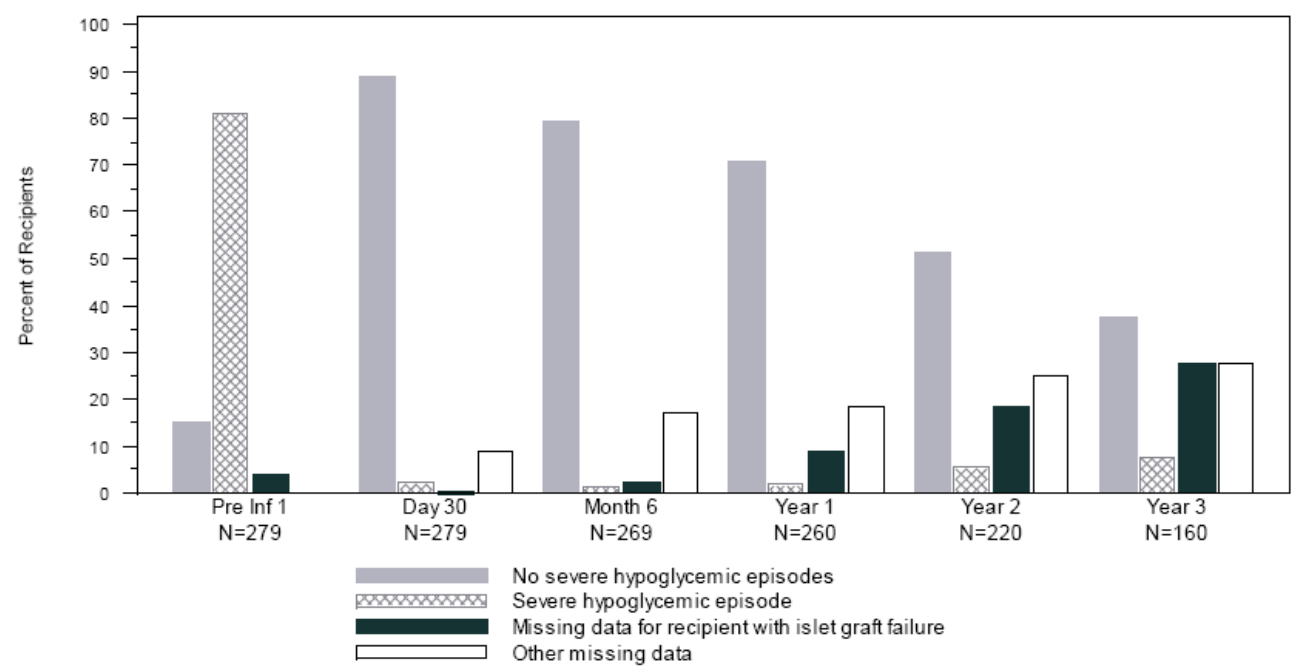

Figure 3. Prevalence of hypoglycemic episodes in patients after their last islet infusion (CITR, 2008).

The intraportal location, while providing ready access to the blood-flow, also exposes the islets to high concentrations of toxic immunosuppressive drugs (Shapiro, 2005) and has been shown to increase the risks of thrombogenesis by the blood-mediated inflammatory reaction to the implanted islets, resulting in the immediate loss of half the grafted islet mass (Eich, 2007). 


\subsubsection{Subcutaneous Implantation}

The subcutaneous space overcomes the issues of islet retrievability and is ideal for implantation and monitoring, however the subcutaneous space is also poorly vascularized (Jansson, 2002; Perez, 2009). Studies performed into the pre-vascularization and concurrent vascularization of the subcutaneous space are widely published (Hiscox, 2008; Liu, 2008; Perez, 2009).

Hiscox et al. (2008) used a system of prevascularized collagen gels to enhance islet cell survival in in vivo subcutaneous studies by maintaining the intraislet endothelial cell structures. Islets placed within the prevascularized structures maintained their ability to produce insulin longer than controls and produced more insulin with fewer IEQ, while remaining responsive to external stimuli. Insulin itself has been shown to induce angiogenesis, through the stimulation of human macrovascular endothelial cell migration and tube formation, independent of vascular endothelial growth factor (VEGF) signaling (Liu, 2008).

Perez et al. (2009) investigated the subcutaneous implantation of islets within plasma-fibroblast gel scaffolds. The use of such a readily biodegradable material provides an initial post-transplant support matrix while enabling the development of a capillary bed and promoting the recovery of extracellular matrix (ECM) interactions through natural tissue remodeling (Stock, 2001). Culture in 3D fibrin gels has also been shown to improve in vivo islet function and in vitro islet survival and differentiation (Amory, 1988; Brendel, 1994; Beattie, 2002).

The plasma-fibroblast gel system of Perez et al. (2009) has only been implanted in a nude mouse model to date, but shows promise. Their gel incorporated 3000 islet equivalents (IEQ) and 6.0$7.5 \times 10^{4}$ fibroblasts in a $4 \mathrm{~mL}$ plasma gel, all components isolated from Wistar rats. Upon implantation into STZ-induced diabetic nude mice, non-fasting blood glucose levels and body 
weight returned to non-diabetic levels at a rate similar to sub-kidney-capsule islet transplant, restoring normoglycemia at $3.9 \pm 1.2$ days $( \pm$ SEM) and were maintained for the 60 day test period in all seven animals. When an intraperitoneal glucose tolerance test (IPGTT) was conducted after 60 days, the area under the curve (AUC) values for the fibrin gel implant were lower than those of all other test groups, even the healthy control mice $(\mathrm{p}<0.05)$, indicating active islets able to efficiently clear glucose from the bloodstream. The lower-than-normal AUC values are credited to the xenogenic nature of the implant, as rat islets have different glucose thresholds than mouse islets (Tatarkiewicz, 1999). Histological evaluation of the implant after 60 days showed complete incorporation into the subcutaneous space, complete with vasculature and collagen matrix.

\subsubsection{Summary}

While studies on immunosuppressed patients, including the hepatic infusions of Shapiro et al. and the subcutaneous transplantations of Perez et al. are promising, their systems will need to be modified to work in a non-immunosuppressed allogenic model before they can be broadly implemented. Additionally, the process of immunoisolation necessitates the recreation of the physical and chemical microenvironment within the immunoisolated system in order to maintain islet viability and function. 


\section{Chapter 2}

\section{Literature Review}

\subsection{Islet Immunoisolation}

The history of graft rejection and toxicity of traditional medicinal systemic immunosuppression techniques previously outlined led researchers to investigate the use of immunoisolation to enhance the efficacy of transplant techniques. The most common immunoisolation technique, microencapsulation, has a proven ability to protect cells from the majority of the host immune response (Lim, 1980; Orive, 2004; Zimmermann, 2005). By eliminating the need for expensive and possibly debilitating immunosuppression regimes, microencapsulation and immunoisolation in general open the use of a tissue-engineered endocrine pancreas system to a much wider group of patients.

When initially proposed, the goal of immunoisolation was to use membranes with a very fine pore size to allow the diffusion of nutrients, wastes and hormones, while preventing all contact with the host immune system. However, studies into alginate membranes with molecular weight cut-offs larger than immunoglobulin $\mathrm{G}(\sim 150 \mathrm{kDa})$ have proven successful, indicating that blocking antibodies and cytokines may not be a requirement of immunosuppression (Omer, 2005). It is believed that the main purpose of the semi-permeable membrane is to act as a physical barrier, preventing antigen recognition by the host $\mathrm{T}$ cells. Solutions to low-molecular weight antigen shedding from within the capsules and interleukin-1 $\beta$ diffusion into capsules continue to be investigated (de Vos, 2006A; Dugas, 2009). 


\subsubsection{Microencapsulation}

In 1964 Thomas Chang introduced the use of semipermeable microcapsules for the encapsulation of enzymes, along with a brief mention of the potential for cellular encapsulation. The publication recognized as the primary inspiration of modern encapsulation research was that by Lim and Sun in 1980 for their study of the encapsulated pancreatic islets within calcium alginate microcapsules.

Microencapsulation is now used for a wide variety of applications as there are many benefits over other geometries, including a high surface area to volume ratio, high resistance to mechanical stress, relatively short diffusion paths between the islets and the surface, leading to improved response rates, and access to various implantation sites (de Vos, 2006B). The process of immunoisolation in microcapsules has an additional benefit, particularly if the cells have to be cultured for extended periods of time. Apoptotic and necrotic islets dissociate and are removed by medium changes in vitro, artificially lowering the detectable levels of dead cells. All encapsulated islets however, are retained within the culture system, providing an accurate measure of viability, and greatly reducing or eliminating the immune-response associated with the release of allogenic and xenogenic cellular debris (Robitaille, 2003).

The most widely implemented microcapsule for islet immunoisolation is the alginate-poly-aminoacid-alginate (APA) system (de Vos, 2006C). The first step involves the encapsulation of the islets in ionically crosslinked alginate beads produced through a variety of methods, most often an assisted dropwise extrusion into calcium chloride. The second step is incubation in a solution of cationic poly-amino acid, commonly poly-L-lysine (PLL) or poly-ornithine (PLO) which associates with the anionic alginate beads, forming a well defined semi-permeable membrane. 
The final step is to re-incubate in a dilute alginate solution, again ionically associated, to reduce the immunogenicity of the cationic poly-amino acid membrane (Hallé, 2009).

Barium chloride may also be used to produce stronger capsules with less susceptibility to dissolution in growth media over extended periods of in vitro culture (Zekorn, 1992). As this study was a 21 day in vitro culture in uncoated alginate particles with assays that required significant capsule manipulation, barium chloride was selected to form the ionic alginate gel. The capsules were left uncoated as the focus was on the interaction between the islet and the matrix, as opposed to the capsule and its surroundings.

The passive diffusion of nutrients and wastes within alginate microparticles necessitates short diffusion pathlengths. The rule of thumb is that no islet should be more than $200 \mu \mathrm{m}$ from the surface of the particle as that is the maximum distance for effective oxygen and nutrient diffusion in most tissues (Mueller-Klieser, 1982). In the Hallé lab, along with others, this has been interpreted with an additional safety factor, such that the average particle should be no larger than $300 \mu \mathrm{m}$, allowing for variations about the mean while staying safely within the maximum $200 \mu \mathrm{m}$ pathlength for even the most central cells of the islet. This fine size necessitates a refined particle production technique.

Simple dropwise extrusion from a needle into a bath of divalent ions $\left(\mathrm{Ca}^{2+}\right.$ or $\left.\mathrm{Ba}^{2+}\right)$ relies on gravity to overcome the surface tension of the droplet at the tip of the needle, producing particles with diameters approaching $2000 \mu \mathrm{m}$ (Poncelet, 2009). The reduction in particle diameter (d) is a function of the cube root of external needle diameter $\left(\mathrm{d}_{\mathrm{e}}\right)$, surface tension $(\gamma)$, and solution density ( $\rho)$ as outlined in Equation 1 (Poncelet, 2009). 


$$
d=\sqrt[3]{\frac{6 d_{e} \gamma}{g \rho}}
$$

Therefore, when optimizing particle diameters from an aqueous solution of alginate with relatively fixed density and surface tension, the practicality of reducing the external needle diameter is limited, and another approach must be investigated.

\subsubsection{Electrostatic Encapsulation}

Electrostatic-mediated droplet production is effective, cytocompatible, and introduces no additional sources of contamination. In this technique, islets are suspended in a dilute $(1-4 \%)$ alginate solution which is dispensed through a needle charged with $\sim 11 \mathrm{kV}$ of potential generated by a high voltage power source. The divalent cation collection bath is grounded and placed far enough from the tip of the needle to prevent arcing, but close enough to produce a stable electrical field to guide the droplets to the collection bath. The difference in potential causes an accumulation of charge on the surface of the droplet which reduces surface tension by a factor of $\left(1-U^{2} / U_{c r}^{2}\right)$ where $U$ is the potential applied, $\mathrm{U}_{\mathrm{cr}}$ is the critical potential required to produce a jet of solution, and $U<\mathrm{U}_{\mathrm{cr}}$ (Poncelet 1999; Poncelet 2009). Pulsing the potential at frequencies of $\sim 40 \mathrm{~Hz}$ in a square wave pattern breaks the flow into discrete droplets of uniform size, smaller than those achieved without electrostatic assistance.

While there are many benefits, including cytocompatibility, sterility, and simplicity, one issue with the technique is scale-up. Solution flow rates for $300 \mu \mathrm{m}$ diameter particles are on the order of $\sim 5 \mathrm{~mL} / \mathrm{h}$, and additional needles necessitate additional equipment and collection baths. 


\subsubsection{Emulsion Encapsulation}

The issue with scale-up is addressed by emulsion encapsulation. The technique developed in the Neufeld lab (Poncelet 1992, 1995) uses triggered internal gelation to form discrete ionicallygelled alginate microparticles in a short period of time with no limitations on batch size, as long as adequate mixing is available to sustain a stable emulsion. Like the dropwise methods, particles are formed from a dilute solution of sodium alginate, however it is buffered at neutral $\mathrm{pH}$ and supplemented with ultra-fine insoluble calcium carbonate. One part of the alginate aqueous phase is poured into three parts oil and emulsified. For acellular systems the emulsion is allowed to form for $\sim 15$ minutes. Once the emulsion has stabilized, the alginate is acidified via an aliquot of acetic acid in oil. The acidification reduces the $\mathrm{pH}$ to 6.5 , sufficient to solubilize the calcium carbonate, liberating calcium ions which ionically crosslink the alginate-laden aqueous phase of the emulsion, forming spherical microparticles. For the application of emulsion encapsulation to MIN6 immortalized $\beta$-cells and primary human islet-depleted pancreatic tissue, Hoesli et al. (2011) optimized the system to reduce the duration of the emulsion and acidification phases. It was determined that with the optimized system there was a post-encapsulation islet viability of 90 $\pm 2 \%$ for MIN6 and $71 \pm 4 \%$ for primary pancreatic tissue in $1.5 \%$ calcium alginate beads $757 \pm$ $20 \mu \mathrm{m}$ in diameter.

Aside from the reduced islet viability, another issue with the emulsion system is a wide particle size range. To ensure optimal diffusion kinetics and complete islet encapsulation, a narrower size range is preferred (Robitaille, 2009).

The density distribution of the alginates in internally-gelled particles is different from that of externally gelled particles. Internally gelled particles are more homogenous and the calcium is 
liberated throughout the particle upon acidification. The externally gelled particles rely on the diffusion of calcium or barium into the particle, resulting in a more dense periphery able to withstand higher shear forces (Thu 1997).

\subsubsection{Alginate}

Alginates are polysaccharides composed of unbranched 1-4 linked $\alpha$-L-guluronic acid (G) and $\beta$ D-mannuronic acid (M). Alginates are naturally sourced polymers, derived primarily from the brown algae Laminaria hyperborean, Macrocystis pyrifera, and Ascophyllum nodosum (Smidsrød, 1990). Depending on the structural requirement of the algaes in their native environment, different species will have different G:M ratios, tidal algaes are exposed to strong surf and therefore have high $\mathrm{M}$ content in order to move with the tide, algaes from deeper, highcurrent zones possess a higher $\mathrm{G}$ fraction (up to $90 \%$ ) for structural integrity (Zimmerman, 2005). The organization of the $\mathrm{G}$ and $\mathrm{M}$ monomers within the polymer is highly variable with blocks of G and M interspersed with alternating MG sequences (Smidsrød, 1990). Divalent cations, such as

$\mathrm{Ca}^{2+}$ and $\mathrm{Ba}^{2+}$ bind to $\mathrm{G}$ blocks on neighbouring chains, forming junction zones, leading to an ionic gel. Higher G content alginates produce capsules with high mechanical strength, while high M alginates exhibit elastic properties (Zimmerman, 2005). 


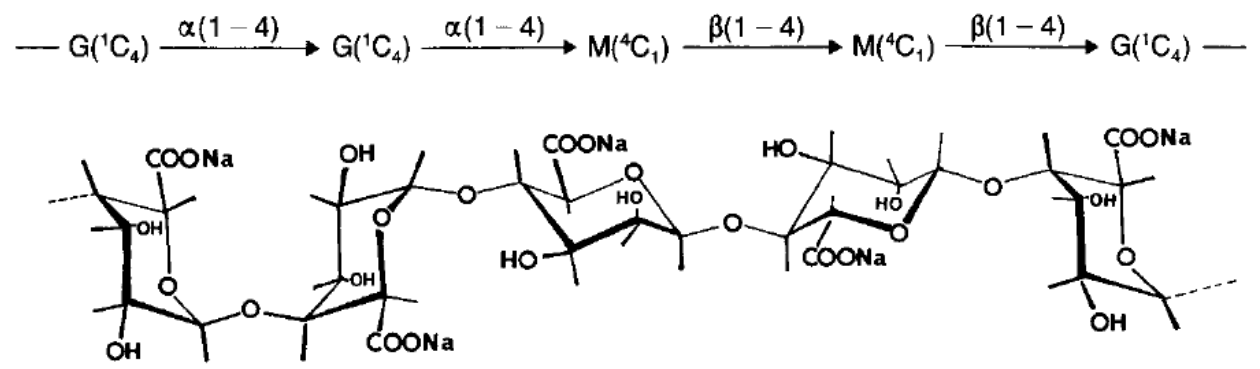

Figure 4. Chemical structure of sodium alginate, composed of guluronic acid (G) and mannuronic acid (M) monomeric units (Smidsrod, 1990).

As alginate ionic gels are stabilized by divalent cations, their mechanical integrity is sensitive to the presence of chelating agents including EDTA, citrate, phosphate and lactate, as well as monovalent cations such as $\mathrm{Na}^{+}$(Smidsrød, 1990).

\subsubsection{Alginate Purification}

The greatest issues with alginate systems to date have been standardization, stability and biocompatibility (Mallet, 2009; de Vos, 2009A).

High-quality alginates are produced from algal stipes collected directly from the sea, peeled and treated with antimicrobial agents to reduce the gram-positive debris which is known to be cytotoxic. Lower-grade alginates are produced from algaes that washed ashore, and have significantly higher contamination levels that are difficult to remove from the processed product. Alginate is extracted from the peeled stipes by EDTA chelation, physical sieving, precipitation with ethanol and $\mathrm{KCl}$ and finally lyophilisation (Zimmerman, 2005).

A recent study by Mallet and Korbutt (2009) investigated the effect of further purification of pharmaceutical-grade alginate on encapsulated murine islet survival in the peritoneal cavity of STZ-induced diabetic mice for a period of 105 days. The islets were encapsulated by 
electrostatic-assisted dropwise addition of $1.5 \%$ alginate solution containing $\sim 10,000 \mathrm{IEQ} / \mathrm{mL}$ into a $\mathrm{CaCl}_{2}$ collection bath. The purified alginate particles had an islet survival rate of $90.5 \%$ relative to $69.2 \%$ for the commercial alginate, with dramatically reduced capsular overgrowth (Figure 5), and superior insulin secretion.

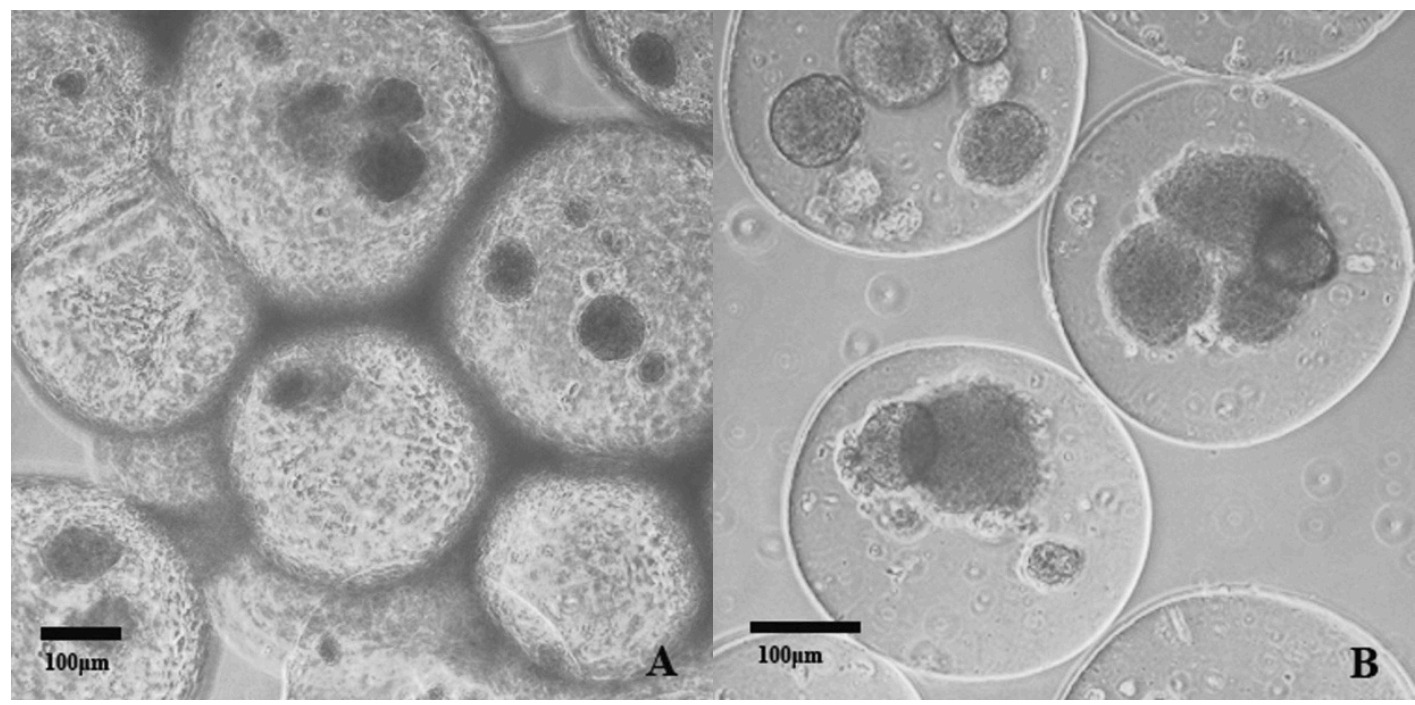

Figure 5. Representative images of dithizone-stained islets encapsulated in nonpurified (A) or purified alginate (B) ex vivo after 105 days in the peritoneal cavity of mice (Mallet, 2009).

A study by Ménard et al. (2010) verified the efficacy of the modified Klöch method of alginate purification in the removal of residual protein contamination via saline dialysis. The dialysis step was incorporated following purification by chloroform extraction, activated and neutral carbon treatment, sterile filtration, acetic acid, sodium citrate, and ethanol extraction. Following dialysis, the purified alginate was precipitated from ethanol and lyophilized. The entire process required 11 days and significant expense to further purify a pharmaceutical-grade starting product. However, the benefits of the modified Klöch method are substantial. A study by Langlois et al. (2009) encapsulated rat islet in purified microparticles and observed significant improvement in insulin secretion and an increase in viability at 14 days from $18 \%$ to $48 \%$. 


\subsubsection{Clinical Trials with Immunoisolated Microcapsules}

In $1996,15,000 \mathrm{IEQ} / \mathrm{kg}$ of neonatal porcine islets were encapsulated in alginate-PLL-alginate capsules and transplanted into the peritoneal cavity of a type I diabetic man without immunosuppression (Elliot, 2007). As porcine and human insulin are similar in nature, differing by only one amino acid, the patient was able to reduce his exogenous insulin dose by up to $30 \%$ at 12 weeks, only returning to pre-transplant levels at week 49. A follow-up study was conducted 9.5 years later by Elliot et al. (2007) to assess the long-term effects of the treatment. Via laparoscopy, nodules were observed throughout the peritoneum and samples were taken for analysis. The capsules contained live islets which stained for insulin and glucagon and the capsules were unchanged in size, but they had become opaque and rigid. The patient claimed to have better glycemic control at 9.5 years, compared to pre-transplant, with a reduction in hyperglycemic episodes and better overnight blood sugar control. The primary conclusion of the follow-up study was that xenogenic islets immunoisolated in alginate-PLL-alginate beads implanted without immunosuppression did not lead to any detectable immune response or fibrosis and achieved partial graft survival for a period of 9.5 years.

With proven success in animal models, immunosuppressed, and xenogenic immunoisolated human clinical trials, clinical trials of allogenic immunoisolated islets were launched. Calafiore et al. (2006A, B) combined the extraction techniques from the Edmonton protocol with the alginatePLO-alginate immunoisolation system to conduct non-immunosuppressed human clinical trials approved by the Italian Ministry of Health, setting standard operative procedures for human islet encapsulation and transplantation. In the first two human trials of the Calafiore protocol (2006A), islets were isolated from donor pancreases, cultured for 24 hours, and encapsulated. The beads 
were formed by the extrusion of $1.6 \%$ endotoxin and pyrogen-free sodium alginate into a $\mathrm{CaCl}_{2}$ bath, with an average bead diameter of $500 \mu \mathrm{m}$ and 1-2 islets/bead. Coatings were formed by incubation in $0.12 \%$ and $0.06 \%$ PLO and finally $0.04 \%$ sodium alginate. The coated beads were injected into the peritoneal cavity under local anesthetic in $150 \mathrm{~mL}$ of saline solution through a 16 gauge needle (400,000 and 600,000 IEQ for patients 1 and 2, respectively). Published results indicate that the patients were unable to eliminate exogenous insulin therapy, but had improved glycemic control at one year, with an elimination of weekly hypoglycemic episodes, and reported no adverse side effects. However, follow-up articles on the study indicating the performance of the other 8 subjects in the 10 member study are noticeably absent.

A publication by Tuch et al. (2009) outlined a four-member clinical trial for the implantation of barium-alginate encapsulated islets by intraperitoneal infusion of $340 \mu \mathrm{m}$ beads into subjects with type I diabetes. The clinical trial was largely unsuccessful, with no change in insulin requirements or glycemic control upon injection of approximately 180,000 IEQ on up to four separate occasions. One subject, who received four infusions, had detectable C-peptide levels for 2.5 years, but experienced no benefits from the infusion. Upon laparoscopy, mimicking a follow-up study by Elliot et al. (2007), it was discovered that the capsules were intact, but contained necrotic islets and were completely encased in fibrous overgrowth.

The variability between clinical trials and between animal and human studies illuminates an issue in the field of islet microencapsulation, due partly to the lack of uniformity in material purification and characterization. 


\subsubsection{Other Immunoisolation Techniques}

In addition to microencapsulation, a number of other techniques have been proposed for the immunoisolation of islets. Although these techniques were not investigated within this body of research, they provide a standard against which the benefits and detriments of microencapsulation can be compared. The wide range of techniques can be roughly divided into intravascular and extravascular devices.

\section{Intravascular Immunoisolation Devices}

Intravascular devices were of considerable interest in early islet studies as they solved the problem of ischemic conditions outside the vasculature by placing islets within semi-permeable membranes in contact with the systemic circulation. Many devices, such as that used by Monaco et al. (1991) were synthetic devices grafted directly to the vasculature. While proving successful in large animal models, including the induction of normoglycemia in dogs for up to a year (Monaco, 1991), the constant risk of thrombosis required severe systemic anticoagulation regimens. Despite the anticoagulation measures taken, thrombosis continued to be an issue, along with complications associated with vascular prosthetic surgery, and infection (de Vos, 2009B). In addition, the PTFE used to construct the devices was shown to induce the production of interleukin-1 beta (IL-1 $\beta$ ), a cytokine which is lethal to islets (Sandler, 1994). Intravascular devices also require more invasive procedures with higher risks of both short term and long term complications.

With the reduction in particle size, the possibility of infusing the hepatic portal vein with encapsulated islets has been investigated. Ten thousand particles smaller than $315 \mu \mathrm{m}$ can be 
infused with only a short and moderate increase in portal vein pressure immediately following injection, returning to normal levels within two hours (Leblond, 1999). In comparison, the same number of particles at $420 \mu \mathrm{m}$ in diameter caused a considerable increase in portal vein pressure, resulting in death within 3 hours for 2 of the 3 rats.

\section{Extravascular Immunoisolation Devices}

Due to the risks associated with vascular anastomosis, recent work in immunoisolation has been focused on extravascular approaches, focusing primarily on microcapsules. In addition to microcapsules, macrocapsules and microcontainers have also been investigated.

Macrocapsules containing large numbers of islets have the advantage of easy retrievability and minimally invasive implantation. The production of macrocapsules from hydrogels has increased their biocompatibility by reducing interfacial tension, protein absorption, and cell adhesion, relative to the older system of polyacrilonitrile and polyvinylchloride diffusion chambers (de Vos, 2002). A recent study by Muthyala et al. (2011) encapsulated mouse islets grown on a crosslinked gelatin matrix within a poly(urethane)-poly(vinyl pyrrolidone) macrocapsule $18 \pm 7$ $\mathrm{mm}$ in length, $4.0 \pm 0.5 \mathrm{~mm}$ in diameter and $1.0 \pm 0.2 \mathrm{~mm}$ in thickness. An in vivo study in rats achieved euglycemia after 15 days, which was sustained for up to 90 days. In the absence of the gelatin scaffold, islet death was observed within 45 days. Macrocapsules suffer from nutritional limitations, necessitating a cell volume fraction of $<10 \%$ to guarantee adequate feeding (de Vos, 2002).

A technology being developed at the University of Texas at Dallas by Gimi et al. (2009) is the use of microcontainers to entrap islets. The containers are $300 \mu \mathrm{m}$ cubes composed of epoxybased polymer SU-8 with micropatterned porous panels containing arrays of $25 \mathrm{~nm}$ wide slots. 
Other unpublished work includes the incorporation of magnetic lids to allow the entrapment of islets without the use of harsh physical or chemical stimuli. Studies with murine islets in vitro have shown no deleterious effects on islet viability and metabolic function. With improvements in the field of micromachining, and continued research by Gimi et al., this approach has the potential to make considerable gains in the coming years.

All of the immunoisolation techniques summarized above aim to increase the viability of the entrapped islets by protecting them from threats originating outside the microenvironment, primarily the immune response. However, the long-term viability and productivity of the islets is also dependent on conditions within the system, and how well they mimic a physiological microenvironment.

\subsection{ECM Incorporation into Islet Culture}

There is evidence that islet structures, while remaining intact, are damaged by the enzymatic treatment of the pancreas. The enzymatic treatment and washing protocols strip islets of ECM components and growth factors, breaking the cell-ECM integrin connections, leading to a state termed anoikis, derived from the Greek word for homelessness (Thomas, 1999). When islets are in a state of anoikis, insulin production is down-regulated (Robitaille, 2003), the expression of integrins $\alpha 3, \alpha 5, \alpha \mathrm{V}$, and $\beta 1$ is decreased (Wang 1999), angiogenesis is disrupted (Perez, 2009), and apoptosis rates increase (Ileva, 1999). In a study on islet apoptosis, Rosenberg et al. (1999) observed an increase in canine islet apoptosis from $5 \%$ to $60 \%$ within the first 5 days of culture. Before elaborating on the use of ECM proteins, specifically collagen and its derivatives, as a tissue engineering matrix, it is important to first investigate the properties of collagen which have lead to its central role in vertebrate physiology. 


\subsubsection{Collagen in the Body}

Collagen is a prominent constituent of the ECM, and the most abundant protein in the human body, comprising $20-30 \%$ of all proteins and over $90 \%$ of the dry weight of some tissues (Harkness, 1961; Badylack, 1995). In the past, it was believed that the ECM was merely a structural framework, however it is now known that the ECM plays an important role in promoting cell adhesion, migration, proliferation, differentiation, and apoptosis (Martins-Green, 1997). The complex physical and chemical messages relayed from the microenvironment through the integrin-mediated connections to the cytoskeleton also regulate gene expression and therefore, cell phenotype (Lutolf, 2005).

'Collagen' is a term which is applied to a superfamily of polypeptide molecules synthesized primarily by mesenchymally-derived cells such as fibroblasts, myofibroblasts, osteoblasts, chondrocytes, and mesenchymal stem cells (Horwitz, 1999; Rosso, 2005). The defining characteristic of the collagen superfamily is the inclusion of at least one triple helical domain with a repeating $(\mathrm{Gly}-\mathrm{Y}-\mathrm{X})_{\mathrm{n}}$ amino acid structure, where $\mathrm{X}$ and $\mathrm{Y}$ can be any amino acid. The righthanded triple helical structure, as shown in Figure 6, is due primarily to hydrogen bonding, steric hindrance between amino acid functional groups, and the small size and hydrophobicity of glycine, which is shielded within the core of the molecule (Hulmes, 2008). 

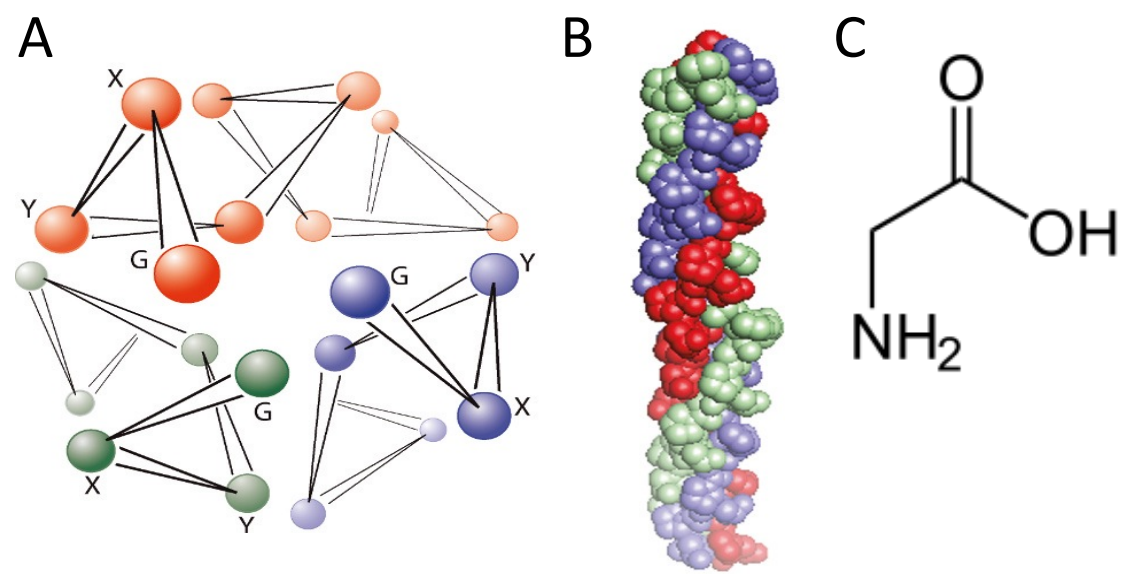

Figure 6. $(A, B)$ Triple helical conformation of collagen molecule $(G l y-X-Y)_{n}$ illustrating the shielding of glycine $(G)$ in the central core of the helix. (C) Chemical structure of glycine (Hulmes, 2008).

The three polypeptide chains, termed $\alpha$ chains, which form the collagen molecule are composed of 337-343 (Gly-X-Y) repeats, depending on the variety of collagen. The $\alpha$ chains assemble into a rod-shaped collagen molecule $300 \mathrm{~nm}$ in length and $1.5 \mathrm{~nm}$ in diameter with a mass of approximately $300 \mathrm{kDa}$ (Hulmes, 2008). The standard image of a triple helical structure applies in varying degrees to the many different collagens which have been identified and placed in the collagen superfamily. There are currently 28 known collagens, which can be categorized into subfamilies including, but not limited to, fibrillar collagens, fibril associated and related collagens, and basement membrane and associated collagens (Ricard-Blum, 2005; Kadler, 2007). Collagen type I falls within the fibrillar collagen subfamily and is the most prevalent collagen in mammalian physiology (Hulmes, 2002).

Due to their $(\mathrm{Gly}-\mathrm{X}-\mathrm{Y})_{\mathrm{n}}$ structure, collagens contain a large quantity of glycine. The amino acid composition of gelatin produced from the hydrolysis of porcine skins (predominantly collagen I and III) is outlined in Table 3. 
Table 3. Amino acid composition of gelatin derived from porcine skins $( \pm 0.05 \%)$ (Bertoni, 2006)

\begin{tabular}{ll|ll}
\hline Amino Acid & $\mathbf{\%}$ & Amino Acid & $\mathbf{\%}$ \\
\hline Gly & 32.7 & Lys & 3.2 \\
\hline Pro & 15.6 & Val & 2.6 \\
\hline Ala & 12.4 & Thre & 2.3 \\
\hline Glu + Gln & 7.9 & Phe & 1.7 \\
\hline Asp & 5.9 & Ile & 1.0 \\
\hline Arg & 5.7 & His & 0.5 \\
\hline Ser & 4.3 & Tyr & 0.4 \\
\hline Leu & 3.7 & Orn & 0.1 \\
\hline
\end{tabular}

In addition to glycine, many other amino acids act as key components of collagen stabilization and crosslinking. The stability of the triple helix is due primarily to the proline and hydroxyproline concentration, which may be as high as $20 \%$ in fibrillar collagens. Proline increases the stiffness of the collagen backbone due to the cyclic nature of the side chain (Figure 7) which sterically hinders movement about the peptide bond. This steric hindrance from proline is also the primary reason $\alpha$ chains exhibit a left-handed helical shape. Hydroxylation of proline by proline hydroxylases further stabilizes the system, raising the degradation temperature of collagen by as much as $30^{\circ} \mathrm{C}$ (Hulmes, 2008). The additional stability imparted by the hydroxylation of proline is believed to be due to increased hydrogen bonding, and the inductive effect of the hydroxyl group which stabilizes the trans-configuration of the peptide bond required to form the triple helix (Brodsky, 2005). 


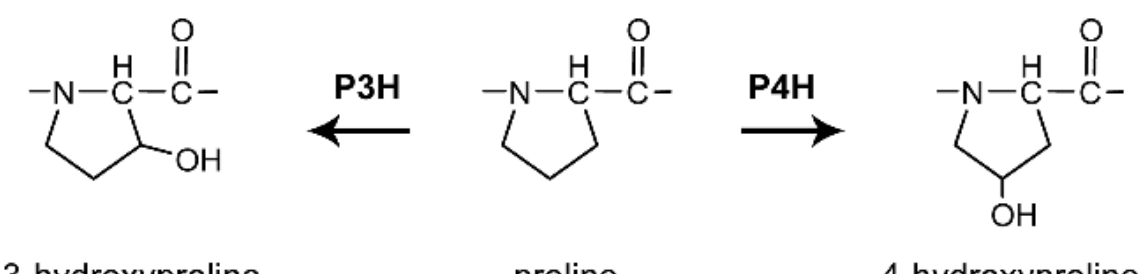

Figure 7. Cyclic structure of proline and hydroxyprolines synthesized by proline hydroxylases (Hulmes, 2008).

\subsubsection{Collagen in Tissue Engineering}

Reconstituted collagen is a popular biomaterial as it is the predominant structural protein found within the human body, it supports cellular adhesion, proliferation, and is only mildly antigenic (Glowacki, 2007). Cell adhesion is due to amino acid sequences within the collagen molecule which are recognized by integrin receptors on cells. The classic example is the RGD (arginineglycine-aspartic acid) sequence which is recognized by the $\alpha 2 \beta 1$ integrin receptor (Yamamoto, 1994). The mild antigenicity of collagen is primarily due to the telopeptide regions, as the triple helical domains are highly conserved between individuals and mammalian species (Thomas, 2005).

Fibril-forming collagens (ex. collagen I) dissolved in cold, low-pH aqueous media will spontaneously form banded fibrils when raised to physiological $\mathrm{pH}$ and temperature (Williams, 1978). The fibrils formed by collagen range between 50 and $200 \mathrm{~nm}$ in diameter. The networks that form are typically $0.1-0.5 \%$ protein by weight, much lower than physiological densities ex. $>5 \%$ of skin (Nakagawa, 1989; Tranquillo, 1999).

The regeneration of an organized fibrillar form is hindered by the bond cleavages and proteolytic degradation necessitated to solubilize the native collagen matrix, resulting in weaker mechanical properties, thermal instability and increased susceptibility to further proteolytic degradation (Lee, 
2001). The extent of spontaneous fibril formation is therefore dependent on the method used to isolate the collagen. Acid-solubilized collagen contains intact telopeptides which facilitate the formation of long cylindrical fibrils. In contrast, when collagen is solubilized by the protease pepsin, the telopeptide regions are cleaved, leaving only the triple helical domains. The resultant product, termed atelocollagen, spontaneously produces fibrils with reduced symmetry and size (Kadler, 1996). However, these same telopeptide regions are the main antigenic region of collagen, resulting in atelocollagen exhibiting a less reactive immune response (Thomas, 2005).

Collagen tissue engineering matrices are remodeled by cells as all metabolic products occur naturally in the body and can therefore be fully utilized. Initially, only a small portion of the collagen is metabolized $(<5 \%)$, however over a period of weeks, up to $80 \%$ may be degraded by cells (Nakawaga, 1989; Guidry, 1985). Depending on the cell type seeded and the location of implantation, concurrent with matrix reorganization and degradation, additional ECM components such as proteoglycans and glycosaminoglycans are synthesized and incorporated into the matrix (Huang, 1993).

\subsubsection{Gelatin in Tissue Engineering}

Gelatin has a long history in the field of biomedical polymer science. It is widely used as an encapsulant in the pharmaceutical industry, as well as in substrates for wound dressings and tissue engineering (Bigi, 2002). Gelatin is the hydrolyzed form of collagen extracted industrially from bovine and porcine skin and bones. Although insoluble in common organic solvents including ethanol, chloroform, ether, benzene, and acetone, gelatin is soluble in glycerol, acetic acid, and water. Aqueous gelatin solutions $>0.5 \%$ spontaneously undergo a sol-gel transition when cooled below $35^{\circ} \mathrm{C}$ (Figure 8), forming a thermally reversible hydrogel (Fakirov, 2007). 
The triple-helical regions formed by the sol-gel transition are connected in a random fashion, as opposed to the highly organized collagen triple-helices (Crescenzi, 2002).

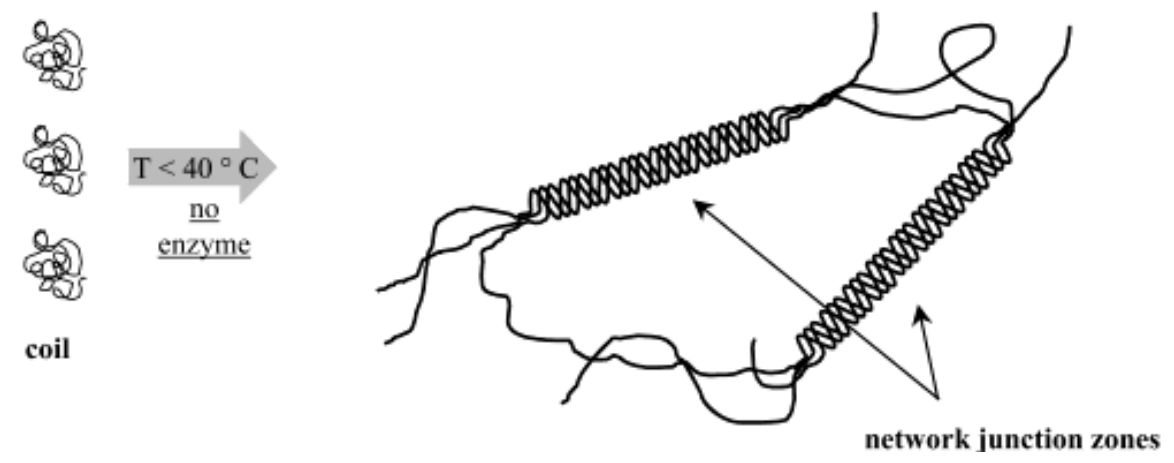

Figure 8. Sol-gel transition of gelatin upon cooling (Crescenzi, 2002).

The glass transition temperature of gelatin is highly dependent on the degree of hydration, ranging from $217^{\circ} \mathrm{C}$ for pure gelatin to an approximate value of $-125^{\circ} \mathrm{C}$ for very dilute gelatin. In the working range for tissue engineering gelatin hydrogels $(<20 \%)$, the glass transition temperature is sufficiently low $\left(<-100^{\circ} \mathrm{C}\right)$ to ensure flexibility is maintained (Fakirov, 2007).

The physical properties of gelatin varieties are commonly described by two parameters, the type, and the Bloom number. The type refers to the preparation method while the Bloom number refers indirectly to molecular weight. Gelatin is produced by boiling collagen-rich tissues in either acidic (type A) or basic (type B) solutions in order to hydrolyze the collagen, rendering it water soluble. The preparation method imparts characteristics to the gelatin, which are summarized in Table 4 (Sigma Aldrich, 2010). Type B cannot be used for applications that require crosslinking via glutamine residues, as they are hydrolyzed during alkaline extraction (Crescenzi, 2002). 
Table 4. Properties of Gelatin A and B

\begin{tabular}{lll}
\hline Property & Type A & Type B \\
\hline Extraction conditions & Acid & Base \\
Isoelectric Point $(\mathrm{pI})$ & $7.0-9.0$ & $4.7-5.2$ \\
Free carboxyl groups $(\mathrm{mmol} / 100 \mathrm{~g})$ & $78-80$ & $100-115$ \\
$\mathrm{pH}$ of $1.5 \%$ solution at $25^{\circ} \mathrm{C}$ & $3.8-5.5$ & $5.0-7.5$ \\
\hline
\end{tabular}

The Bloom number is a measure of the force required to depress a standardized sample of gelatin by $4 \mathrm{~mm}$. Low Bloom numbers (50-125) have an average molecular weight of 10-25 kDa, while high Bloom gelatins (225-325) have an average molecular weight of 50-100 kDa (Sigma, 2010). Low Bloom gelatins result from the cleavage of peptide bonds in the $\alpha$ chains during extraction (Fakirov, 2007). Low Bloom gelatins $(<20 \mathrm{kDa})$ don't possess sufficient chain length to form physical crosslinks or adequate chemical crosslinking densities to gel (Crescenzi, 2002). The most commonly used commercial gelatin in the literature is 300 Bloom Type A which corresponds to an average molar mass of approximately $80 \mathrm{kDa}$ (Sigma, 2010).

Due to the random-coil configuration of gelatin, reaction sites are less sterically hindered compared to triple helical collagen molecules. This may explain why gelatin has been shown to achieve higher degrees of synthetic crosslinking than collagen (Ciardelli, 2010).

\subsubsection{Pancreatic Islet ECM Composition}

The ECM is specifically tailored to the microenvironment required for each cell type and their physiological function. The ECM microenvironment in the periphery of adult islets is poorly defined in the literature. One recent review by Cheng et al. (2011) indicated that collagen I, II, III, IV, V, VI, laminin, fibronectin, elastin, nidogen/entactin, and vitronectin were important components of the pancreatic islet ECM, in conjunction with the heparin sulfate and chondroitin sulfate proteoglycans perlecan, syndecan, glypican, betaglycan, agrin, and versican. The 
interfaces between islets and ECM proteins are complex, with both integrin and non-integrin interactions. Non-integrin discoidin receptors for collagens I-V are found specifically in the endocrine tissue and have been shown to regulate adhesion, migration, differentiation, proliferation, and ECM production. One interesting trend observed in mice was the absence of perlecan in the diabetic mouse pancreas despite strong staining in the healthy mouse pancreas, the causation or correlation of this observation has not been confirmed (Irving-Rodgers, 2008). Laminin-332 is found specifically in endocrine cells in vivo and is produced by purified islets in vitro, with increased production under interleukin-1 $\beta$-induced stress conditions (Armanet, 2009). The form of the ECM capsule surrounding islets differs amongst mammalian species, being continuous in canine islets, intermediate in rat and human islets, and sparse in porcine islets, reminding us that the composition and structure of mammalian islets differ, hindering the ability to infer clinical outcomes from non-human investigations (van Deijnen, 1992). A deeper understanding of the islet ECM composition would allow for a more directed approach towards improving islet viability and function. Despite the lack of a solid foundation in islet ECM composition, many studies have been performed using ECM components in addition to collagen I and gelatin that have proven effective in other tissue engineering applications.

\subsubsection{Other ECM Proteins and Growth Factors}

Weber et al. (2008) investigated the incorporation of collagen I, collagen IV, fibrinogen, fibronectin, entactin-free laminin, and vitronectin into poly(ethylene glycol) (PEG) hydrogels containing immortalized MIN6 $\beta$-cells at 50,000 cells per $30 \mu \mathrm{L}$ sample. All ECM-containing gels showed statistically significant $(\mathrm{p}<0.05)$ increases in $\beta$-cell survival and corresponding decreases in apoptosis over PEG controls. Gels containing collagen IV and laminin also displayed 
increases in insulin production in response to glucose stimulation, with the best result achieved through a combination of $25 \mu \mathrm{g} / \mathrm{mL}$ collagen IV and $75 \mu \mathrm{g} / \mathrm{mL}$ laminin.

Laminin is an important ECM protein in the development of a physiological microenvironment for pancreatic islets, as it is one of the main components of the pancreatic islet basement membrane (Kawazoe, 2009). Laminin is also the only ECM component shown to maintain islet structural integrity and insulin content distribution within human islets in 2D culture (Daoud, 2010).

Issues have been raised in regards to naturally sourced collagen and gelatin, including batch-tobatch variability, pathogen transmission, and immunogenicity (Serban, 2008). For this reason, recombinant collagens and gelatins have been synthesized. The development of recombinant collagens has been thoroughly reviewed in the literature from a number of different perspectives (Olsen, 2003; Ruggiero, 2008; Xu, 2010).

Recognizing that the best way to reproduce the pancreatic microenvironment was to use pancreatic tissue, DeCarlo et al. (2010) entrapped decellularized pancreatic and liver tissue and rats islets within PVA/PEG tubes. Monitored over 42 days, the insulin release was sustained, but only in the pancreatic ECM. In the liver ECM insulin release was significantly reduced with time, as observed in 2D culture. Therefore, care must be taken to reproduce the native microenvironment with as much detail as possible, instead of lumping everything together as 'ECM'.

Robitaille et al. (2003) and Ileva et al. (1999) discovered that incorporation of insulin-like growth factor II (IGF-II) into barium-alginate microparticles decreased neonatal islet apoptosis and necrosis by $69 \%$ in vitro. In Robitaille's immunoincompetent in vivo murine model, $100 \%$ of the mice with 300 encapsulated IGF-II cultured islet equivalents became normoglycemic, and 75\% 
retained normoglycemia at 143 days. In contrast, the encapsulated non-IGF-II-incubated model reported $92 \%$ normoglycemia, with a drop to only $25 \%$ at day 143 . The non-encapsulated models fared even worse, with none of the non-IGF-II-incubated mice and only one of the IGF-IIincubated mice attaining normoglycemia for a short period of time. These results are promising, as the procedure uses so few islets to restore normoglycemia, roughly $12,000 / \mathrm{kg}$ of body weight. The study even saw limited success at the induction of normoglycemia with only 6,000 islet equivalents/kg in IGF-II supplemented barium-alginate capsules (Robitaille, 2003).

Perez et al. (2009) used fibroblasts to secrete growth factors and native clotted fibrinogen matrices to promote the survival of islets through the induction of angiogenesis and tissue remodeling. The use of fibroblasts to secrete growth factors and deposit ECM allows for the sustained maintenance of the microenvironment over a much longer timescale.

Success in islet implantation likely relies on insulin up-regulation and improved islet viability through the restoration of the physical and chemical microenvironment of the islet utilizing a combination of hormones, growth factors, and ECM components.

\subsection{Islet Growth on Three-Dimensional Matrices}

Previous researchers have dissociated islets into individual cells to homogenously inoculate microporous gelatin microcarriers (Del Guerra, 2001). The gelatin microcarriers provided considerable benefits over the alginate controls, increasing glucose-induced insulin production. However, the implantation of discrete cells from dissociated islets suffers from the elimination of cell-cell interactions found within the aggregated islet state which up-regulate insulin secretion (Bosco, 1997). The resulting insulin production in the microcarriers, while better than the control, was only $60 \%$ that of undissociated islets entrapped in alginate (Del Guerra, 2001). Therefore, 
matrices, while beneficial, must be investigated on a scale that allows the seeding of intact 150 $\mu \mathrm{m}$ diameter islets. To seed a preformed porous matrix, the pores must be 5-10 times the size of the islets to ensure homogenous seeding (Landers, 2002). This renders many traditional matrices, which rely on porogens or lyophilisation, too fragile for practical application to islets. Even with the proper pore sizing, seeding efficiencies of dissociated cells and aggregates are an issue, with some studies achieving only 50\% (Del Guerra, 2001).

Cultures of whole islets on matrices of modified and unmodified poly(lactide-co-glycolide) fibers have shown increased viability and insulin secretion (Lucas-Clerc, 1993; Chun, 2008; Salvay, 2008; Hou, 2009; Kawazoe, 2009). The addition of basement membrane proteins to the surface of these scaffolds, including collagen IV, laminin, and fibronectin have been proven to further increase islet viability and insulin secretion (Jiang, 2002; Hamamoto 2003; Kaido, 2004; Salvay, 2008). To date, all investigations of pancreatic islet culture within fibrous or porous matrices have been of macroscopic slab or matrix geometries.

Daoud et al. (2011) investigated the growth of islets on matrices $8 \mathrm{~mm}$ in diameter and $3.6 \mathrm{~mm}$ thick composed of $150 \mu \mathrm{m}$ thick PLGA strands, producing a highly defined lattice geometries with pore sizes of 260,350 , and $400 \mu \mathrm{m}$. Despite the limitations of geometry necessitated by islet-seeding, these matrices have proven to sustain human islet culture for a period of 10 days while maintaining a fresh-islet architecture and insulin responsiveness superior to collagen gel entrapment or suspension culture. Insulin production and gene expression were upregulated by the inclusion of the scaffold, relative to collagen I gel controls and collagen I gels supplemented with fibrinogen and collagen IV (Daoud, 2011). 
In order to combine the benefits of the discoveries outlined above and achieve the goals of this present study, a matrix of discrete protein fibers was required, produced on a scale that would easily fit within a $300 \mu \mathrm{m}$ microcapsule.

\subsubsection{Production of Protein Fibers - Nanofibers}

A common approach to collagen or gelatin matrix production is the electrospinning of nano-scale fibers which mimic natural collagen fibrils. Gelatin nanofibers can be produced by electrospinning, but only from a few solvents, most of which are highly toxic (Song, 2008). A more benign solvent system of interest is composed of acetic acid, ethyl acetate and water, which has been shown to produce cytocompatible microfibers from $8-11 \%$ gelatin solutions (Song, 2008; Sisson, 2009). Acetic acid is incorporated to acidify the aqueous medium and dissolve the gelatin, in addition to lowering the surface tension of the solution. Ethyl acetate raises the $\mathrm{pH}$ to prevent denaturation of the proteins, while further decreasing the surface tension to facilitate electrospinning. Finally, the addition of water aids in the miscibility of acetic acid and ethyl acetate (Song, 2008).

Due to the high solubility of these materials in aqueous environments, crosslinking is necessitated. The use of multiple crosslinking agents for this purpose has been investigated in two studies.

Sisson et al. (2009) investigated the use of vapour phase glutaraldehyde, genipin and glyceraldehyde in $70 \%$ ethanol, and reactive oxygen species (ROS) from a plasma cleaner to crosslink electrospun gelatin scaffolds. The use of reactive oxygen species in a dry environment leads to the promotion of the degradation reaction over the crosslinking reaction, resulting in the ROS-exposed matrices dissolving within a matter of hours. The other systems worked well, 
maintaining mechanical integrity beyond two weeks. However, the mechanical strength was not quantified. A cytotoxicity study with osteosarcoma cells showed that all scaffolds performed at least equivalently to 2D controls, with glyceraldehyde showing the best cellular response. However, the form of the crosslinked fibers varied substantially, due to the presence of water during the crosslinking process, partially dissolving the substrate, as can be seen in Figure 9. The glyceraldehyde substrate had fused junctions, unlike the ROS substrate. At equivalent concentrations, glyceraldehyde performed better than genipin in the $37^{\circ} \mathrm{C}$ aqueous environment, which was attributed to the slower crosslinking kinetics of genipin.

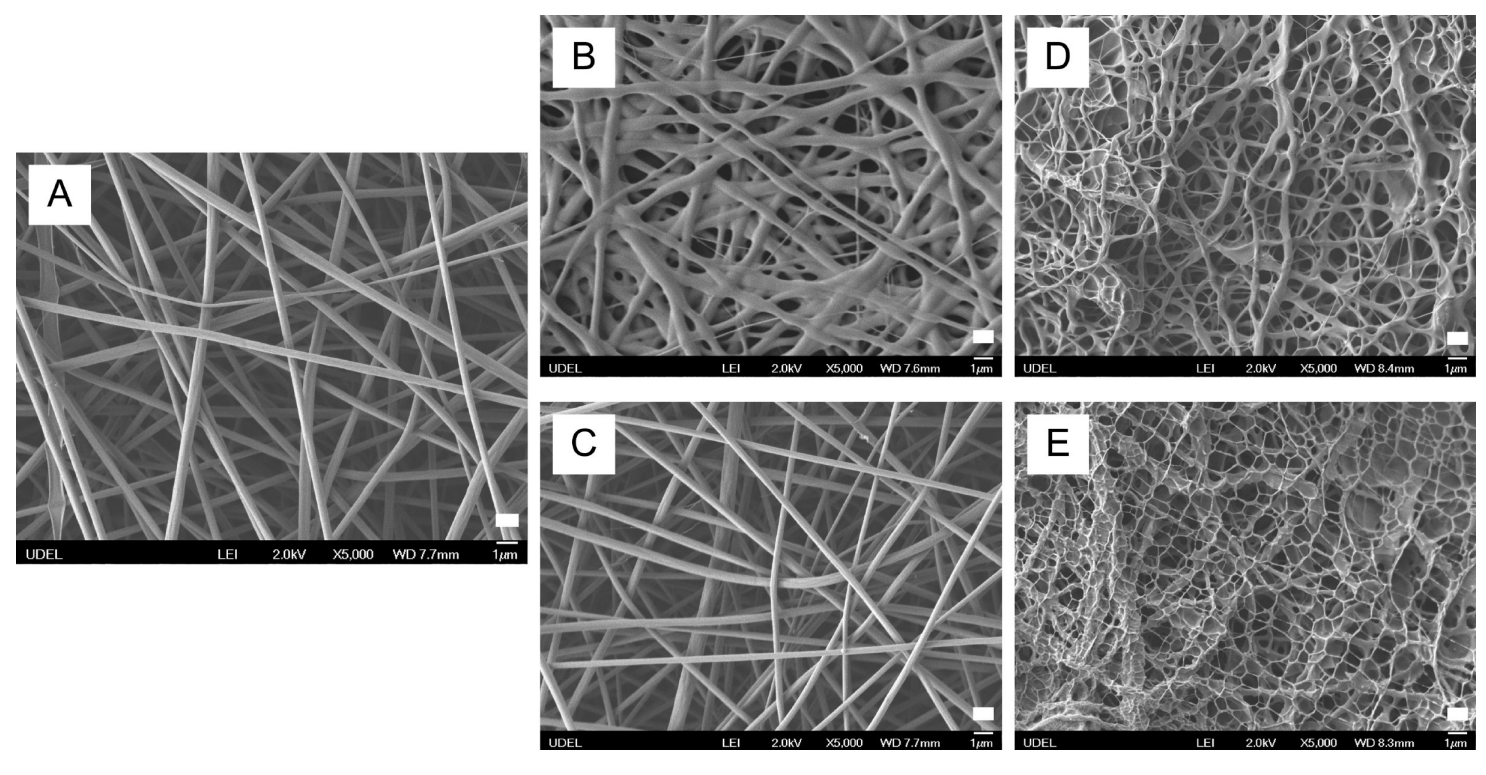

Figure 9. (A) Uncrosslinked gelatin fibers, (B) glutaraldehyde crosslinked (0.5\%), (C) reactive oxygen species crosslinked, (D) glyceraldehyde crosslinked $(0.5 \%)$, (E) genipin crosslinked (1\%). Scale bar represents $1 \mu \mathrm{m}$ (Sisson, 2009).

In a similar study, collagen nanofibers were crosslinked with EDC-NHS, UV, genipin, and transglutaminase and the fibers were analyzed by SEM (Figure 10) (Torres-Giner, 2009). Although only one reagent, genipin, was replicated between the two papers, the very different morphologies observed between the two genipin-crosslinked samples indicate that the difference 
is due to the interplay between substrate solubility and reactivity, as the slower the crosslinking reaction, the greater the change in fiber morphology.
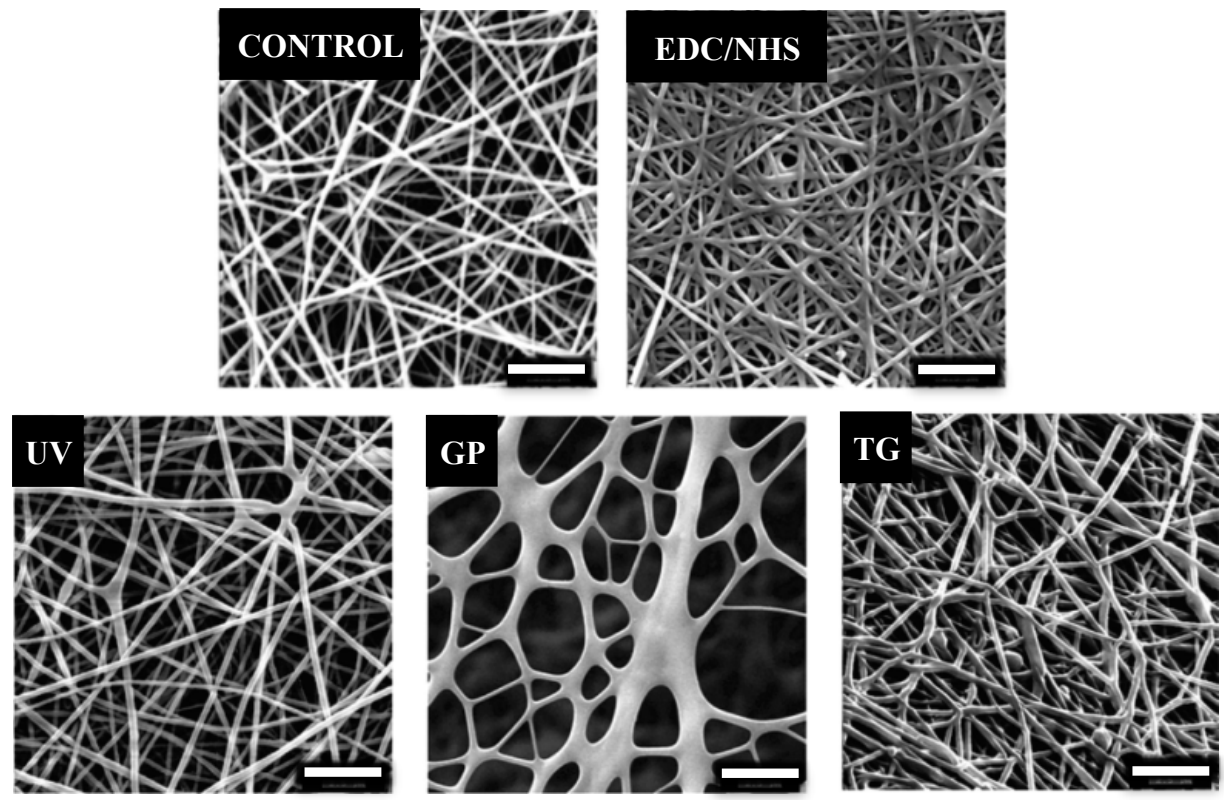

Figure 10. SEM images of uncrosslinked collagen fibers (Control), and fibers crosslinked by EDC/NHS, UV, genipin (GP), and transglutaminase (TG). Scale bar represents $2.5 \mu \mathrm{m}$ (Torres-Giner, 2009).

An innovative method for producing crosslinked electrospun nanofibers in a single step was recently published by Nguyen et al. (2010). Gelatin was dissolved in trifluoroacteic acid (TFA) and an aliquot of glutaraldehyde (GA) was added before electrospinning. Due to the acidic environment, the kinetics of GA crosslinking were hindered. However, when dried overnight in a vacuum oven, the TFA evaporated and the GA was able to effectively crosslink the fibers. This approach bypasses the issue with gelatin solubility in aqueous crosslinking solutions, while simplifying fiber production. 
A nanofiber system can also be produced by the self-assembly of peptide amphiphile (PA) which self-assembles into a nanostructured gel-like scaffold, which has shown promise in rat-islet culture (Zhao, 2009; Lim, 2011).

\subsubsection{Production of Protein Fibers - Microfibers}

The production of gelatin microfibers is difficult due to gelatin having no intrinsic fiber-forming characteristics. Therefore, the fiber formation must be facilitated with a coagulant or a support matrix.

Solutions of $15 \%$ gelatin in dimethyl sulphoxide (DMSO) maintained at $55^{\circ} \mathrm{C}$ can produce discrete fibers when extruded into a $-20^{\circ} \mathrm{C}$ bath of methanol. These fibers have sufficient mechanical strength to be drawn up to sixteen times their original length, orienting the molecules within the fiber, producing triple helical crystalline domains (Fukae, 2008). In the Fukae et al. study, there was no mention of the optimized fiber diameter, simply an outline of the production process, but from their needle diameter and draw ratio, a diameter $\sim 100 \mu \mathrm{m}$ can be estimated. In addition, the concentration of residual DMSO in the fibers is also unreported.

A process developed by Yang et al. (2009) used alginate as a support matrix in order to produce gelatin microfibers from a similar extrusion and drawing process. A solution of $1.5 \%$ alginate and $0.245 \%$ gelatin was extruded into a $2 \% \mathrm{CaCl}_{2}$ collection bath. The collection bath crosslinked the alginate phase, allowing the fiber to be drawn around a mandrel with a draw ratio of up to 3.14. The alginate was extracted with $4^{\circ} \mathrm{C}$ phosphate buffer and the gelatin fibers were crosslinked with glutaraldehyde. The final dry fiber diameters achieved were $181 \mu \mathrm{m}$. 


\subsection{Crosslinking Agents and Cytocompatibility}

Thermally gelled collagen, gelatin, and recombinant collagen matrices lack the mechanical integrity and thermal and proteolytic stability required to produce a viable replacement tissue, as they are simply held together by physical associations, not covalent crosslinks (Lee, 2001; Guilak, 2003). Chemical crosslinking techniques utilize the reactive lysine, glutamic acid, glutamine, and hydroxyl groups which extend from the surface of the molecules (Avery, 2008).

The presence of intermolecular and interfibrillar crosslinks prevents molecules and fibers from sliding past one another under stress, increasing the mechanical strength and the degradation temperature (Torres-Giner, 2009). In addition, the process of crosslinking reduces the flexibility of the molecules and fibers, resulting in decreased proteolytic degradation as the enzymatic attachment domains become increasingly hindered (Avery, 2008). The increase in thermal stability observed with crosslinking is due to the decreased hydration of the fibers resulting from a reduced average axial separation. The change in degradation temperature is therefore only indirectly dependent on the crosslinking agent used, and instead dependent on the degree of hydration obtained under each regime (Miles, 2005).

Many traditional crosslinking techniques taken from industry suffer from cytocompatibility issues. These issues may include toxic residues from residual initiators and catalyst and unreacted or partially reacted crosslinking agents, while other cytocompatible techniques suffer from high cost or scale-up issues (Matsuda, 1999; Sung, 1999). Therefore, progress in collagen and gelatin based tissue engineering requires crosslinking agents and techniques that restore the physical properties of native tissues in a non-cytotoxic yet scalable and cost-effective manner. The 
cytotoxicity of various crosslinking agents used for crosslinked pre-formed acellular matrices and in situ matrix crosslinking are summarized in Table 5.

Table 5. Summary of Crosslink Agent Cytotoxicity

\begin{tabular}{|c|c|c|c|c|}
\hline Agent & Pre-Formed & In Situ & Study & Notes \\
\hline Formaldehyde & High & Complete & Sung, 1999 & $<1 \mathrm{ppm}$ in situ \\
\hline Glutaraldehyde & Minor & Complete & $\begin{array}{l}\text { Sung, 1999; Duan, } \\
\text { 2006; Sisson, } 2009\end{array}$ & $<1 \mathrm{ppm}$ in situ \\
\hline D,L-Glyceraldehyde & None & None & Sisson, 2009 & \\
\hline Carbodiimide & None & Low & $\begin{array}{l}\text { Sung, 1999; Duan, } \\
\text { 2002; Torres- } \\
\text { Giner, } 2008\end{array}$ & $>10,000 \mathrm{ppm}$ \\
\hline Genipin & None & $\begin{array}{l}\text { None/ } \\
\text { Minor }\end{array}$ & $\begin{array}{l}\text { Sung, 1999, 2001; } \\
\text { Sundararaghavan, } \\
\text { 2008; Sisson, } 2009\end{array}$ & $\begin{array}{l}>10,000 \mathrm{ppm}, \\
\text { toxic }>50 \mathrm{ppm} \text { in } \\
\text { presence of polylysine } \\
\text { Significant at } 5 \mathrm{mM}\end{array}$ \\
\hline $\begin{array}{l}\text { X-Ray } / \gamma \quad \text { Radiation } \\
\text { Plasma Treatment }\end{array}$ & - & Complete & $\begin{array}{l}\text { Torres-Giner, } \\
\text { 2008; Sisson, } 2009\end{array}$ & $\begin{array}{l}\text { Most dry irradiated } \\
\text { matrices degrade } \\
\text { quickly }\end{array}$ \\
\hline UV & None & Minor & $\begin{array}{l}\text { Kato, 1994; } \\
\text { Wollensak, 2003; } \\
\text { Ahearne 2008; } \\
\mathrm{Hu}, 2009\end{array}$ & $\begin{array}{l}\text { Dependent on UV } \\
\text { exposure intervals }\end{array}$ \\
\hline Transglutaminase & None & None & $\begin{array}{l}\text { Chen, 2003, 2005; } \\
\text { Bertoni, 2006; } \\
\text { Torres-Giner, } 2008 \\
\end{array}$ & \\
\hline Epoxy Compounds & - & Complete & Sung, 1999 & $<1 \mathrm{ppm}$ in situ \\
\hline
\end{tabular}

\subsubsection{Glutaraldehyde}

Glutaraldehyde is the most commonly used crosslinking agent for collagen and gelatin systems due to the facile nature of the crosslinking process which produces chemically stable bonds and significantly increases the mechanical strength of the system (Covington, 1997; Avery, 2008).

The exact method of crosslinking in a glutaraldehyde system is complex (Figure 11), further complicated by aldol contamination and polymeric complexes produced during storage. 
Glutaraldehyde compounds react with the $\varepsilon$-amino of lysine and rearrange to produce heterocyclic compounds which oxidize to pyridine rings (Sheu, 2001; Avery, 2008).

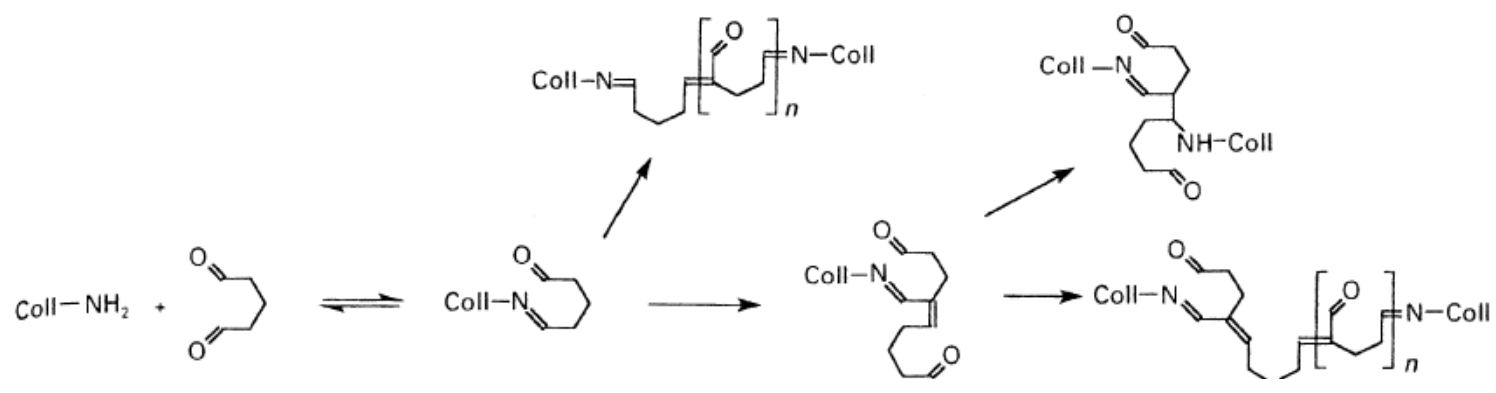

Figure 11. Crosslinking mechanisms of glutaraldehyde and collagen-bound primary amines (Sheu, 2001).

A serious issue with glutaraldehyde is its cytotoxicity. Glutaraldehyde is known to produce cytotoxic environments in the periphery of the implant in addition to long-term calcification (Khor, 1997). Cytotoxicity can be greatly reduced by thorough washing (Sisson, 2009); however, the degradation products of matrix metabolism are ultimately cytotoxic (Zhang, 2006B). Therefore, glutaraldehyde has been largely relegated to use as a control when analyzing the mechanical integrity and cytotoxicity of less toxic crosslinking techniques (Sisson, 2009; Sung, 1999).

\subsubsection{Carbodiimide Chemistry}

Carbodiimide chemistry uses carbodiimides $(\mathrm{R}-\mathrm{NH}=\mathrm{C}=\mathrm{NH}-\mathrm{R})$ to produce zero-length crosslinks between primary amines and carboxylic acid functional groups. The most common carbodiimide system is ethyl-3(3-dimethylamino)propyl carbodiimide (EDC) enhanced by the addition of $\mathrm{N}$ hydroxysuccinimide (NHS). The EDC-NHS chemistry eliminates cytotoxic metabolic products as the crosslink is a simple peptide bond and the NHS reduces the number of potentially toxic side products (Torres-Giner, 2009). 
The zero-length crosslink, while reducing long-term cytotoxicity, suffers from the effects of steric hindrance, which has less of an impact on larger crosslinking molecules. This has been overcome by the use of dendrimers, such as the one shown in Figure 12, which provide multiple amine groups for crosslinking and a large, flexible structure to increase the likelihood of encountering an EDC-activated carboxyl group (Duan, 2006).

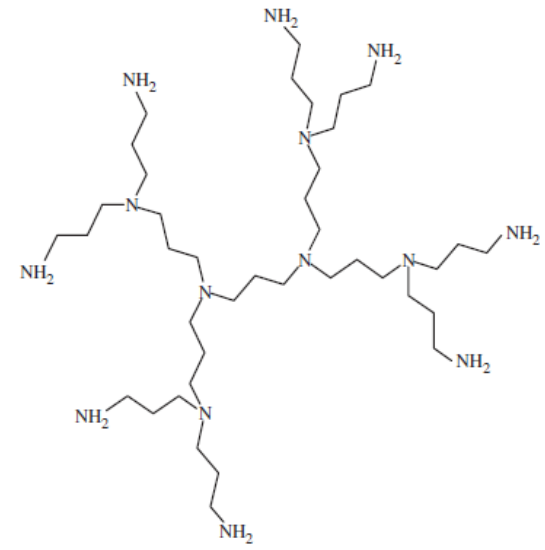

Figure 12. Polypropylene octamine dendrimer (Duan, 2006).

The addition of dendrimers as been shown to greatly increase the mechanical strength of the resultant gels over standard EDC and glutaraldehyde controls, while maintaining a cytocompatible environment (Duan, 2006).

\subsubsection{Radiation/Plasma}

High-energy irradiation (X-ray, $\gamma$-ray) and plasma treatment of collagen and gelatin samples leads to two competing reactions, degradation and crosslinking. It has been determined that the crosslinking reaction is predominant in hydrated systems while the degradation reaction is the primary reaction under dry conditions (Bailey, 1968). The crosslinking reaction proceeds by the 
formation of free radicals in aqueous systems which are highly reactive, forming more crosslinks than chain cleavages.

Under UV irradiation, the primary mechanism is the degradation of phenylalanine and tyrosine due to the energy absorption of their aromatic rings. This degradation results in dihydroxyphenylalanine and dityrosine crosslinks (Kato, 1995; Wollensak, 2003), although this mechanism is contested, as these crosslinks have only been identified in a limited number of studies, and are noticeably absent in others (Miles, 2000). The exact mechanism of the reaction is dependent on oxygen concentration, $\mathrm{pH}$, type of collagen and wavelength of UV light (Avery, 2008).

\subsubsection{Transglutaminase}

Transglutaminases are ubiquitous in nature, found in vertebrates, invertebrates, plants and microorganisms (Chen, 2005). There are eight known mammalian transglutaminases, some of which have been used in biomedical applications for the crosslinking of collagen and gelatin. Mammalian transglutaminases are calcium dependent, a factor which has been used to control the gelation kinetics of gelatin-based systems (Sanborn, 2002).

Microbial transglutaminase (mTG) has been used extensively, as it is calcium independent, has a less-specific substrate specificity, is a smaller molecule, has a higher reaction rate, and is considerably less expensive as it is produced as a food additive (Kashiwagi, 2002). The primary source of mTG for both academic and culinary applications is a line of commercially available products produced by Ajinomoto under the Activa ${ }^{\mathrm{TM}}$ brand from a strain of Streptoverticillium mobaraense (Chau, 2005). As Activa is a commercial culinary product and not a research-grade purified enzyme, there is a large concentration of maltodextrin in the formulation. Some 
researchers feel the need to remove this component (up to $99 \%$ of the dry mass) in order to make independent conclusions about the effects of mTG, while others do not (McDermott, 2004; Chau, 2005).

Transglutaminases catalyze the formation of $\varepsilon(\gamma$-glutamyl)lysine linkages between glutamine and lysine residues in collagen and gelatin systems, as shown in Figure 13 (Chen, 2003; Chau, 2005). The composition of these amino acids within gelatin A are roughly $8 \%$ glutamine and glutamic acid, and 3\% lysine (Crescenzi, 2002; Bertoni, 2006). Of the available substrates, only approximately $20 \%$ undergo crosslinking, forming 2 crosslinks per gelatin chain and 1 crosslink per collagen molecule at saturation (McDermott, 2004; Chau, 2005). In type B gelatin, all glutamine residues have been hydrolyzed by the alkaline extraction and are therefore unable to form crosslinks (Crescenzi, 2002). Transglutaminases can also be used to covalently bind compounds containing primary amines to glutamine, allowing for the facile inclusion of other proteins and surface modifiers (Griffin, 2002).

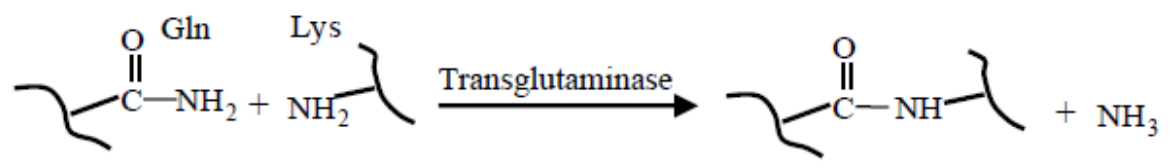

Figure 13. $\varepsilon(\gamma$-glutamyl)lysine linkage formed by transglutaminase (Chen, 2003)

The activity of transglutaminase is defined in terms of units. One unit of transglutaminase will catalyze the formation of $1 \mu \mathrm{mol}$ of hydroxamate per minute from hydroxylamine and N $\alpha$-CBZGlutaminylglycine at $\mathrm{pH} 6.0$ and $37^{\circ} \mathrm{C}$ (Bertoni, 2006). The reactivity of transglutaminase enzymes can be monitored by the formation of ammonia (Bertoni, 2006).

Unpurified Activa $\mathrm{TI}^{\mathrm{TM}}$ has a reported activity of $80-135 \mathrm{U} / \mathrm{g}$. The saturation concentrations for transglutaminases in gelatin, determined by ammonia quantification, are $0.025 \mathrm{U} / \mathrm{mg}$ for tTG and 
$0.05 \mathrm{U} / \mathrm{mg}$ for $\mathrm{mTG}$, with crosslinking generally complete by 10 minutes at $\mathrm{pH} 6.0$ and $37^{\circ} \mathrm{C}$ (Bertoni, 2006). A study by Chau et al. (2005) found that purified mTG produced an almost twofold increase in the amount of isopeptide bond formation over tTG, attributed to its broader substrate specificity and lower molecular weight. Treatment of native collagen with transglutaminase also increased resistance to cell-mediated degradation and significantly increased growth, proliferation, and motility of human osteoblasts (Chen, 2005).

\subsubsection{Genipin}

Genipin is a natural crosslinking agent, food dye, and anti-inflammatory derived from the fruit of Gardenia jasminidides. Genipin reacts with primary amine groups and then bonds with another amine-bound genipin molecule, producing a stable bond with extremely low toxicity (Figure 14) (Sung, 1999; Liang, 2002). Genipin is effective at $\mathrm{pH} 7.4$ at a concentration of 5-10 mM, with increasing kinetics at higher $\mathrm{pH}(8-9)$. Under optimal conditions, the $\mathrm{t}_{1 / 2}$ for genipin crosslinking is 10-18 min (Slusarewicz, 2010). The maximum crosslinking index of the gelatin-genipin system is $75 \pm 2 \%$, obtained at genipin concentrations of $0.5 \mathrm{wt} \%$ and above (Yao, 2004).

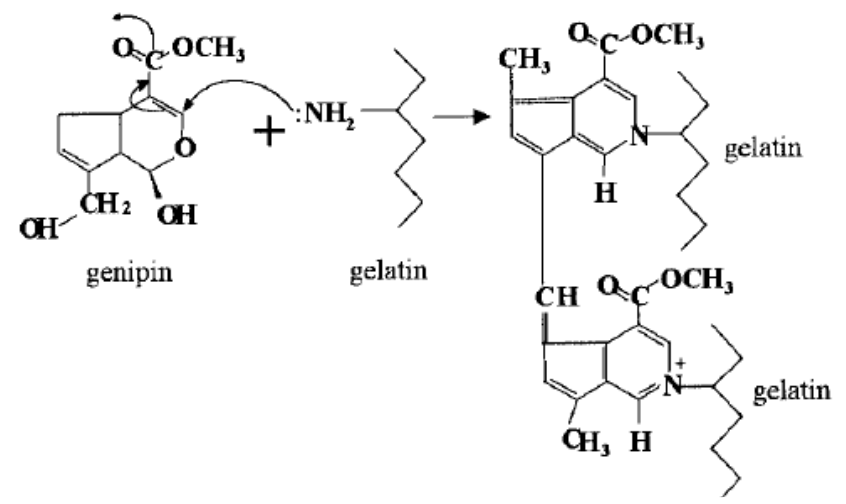

Figure 14. Genipin crosslinking of gelatin (Liang, 2002) 
A study on fibroblasts crosslinked into gelatin-based tissue adhesives found that the genipin matrix was approximately 1000 times less cytotoxic than the glutaraldehyde matrix, with no significant cytotoxicity observed at 10,000 ppm (Sung, 2001). However, in the presence of polylysine (a primary amine-rich dendrimer), cytotoxicity was observed at $50 \mathrm{ppm}$ (Sung, 1999). An in vivo study of acellular genipin-crosslinked gelatin microspheres implanted intramuscularly demonstrated a greatly reduced inflammatory reaction and slower degree of degradation relative to glutaraldehyde crosslinked controls. It was hypothesized that the heterocyclic genipin crosslink induced appreciable steric hindrance into the matrix, interfering with the action of proteases, relative to the linear glutaraldehyde crosslink (Liang, 2002). In the event that the genipincrosslinked gelatin matrices are degraded, studies have shown that the byproducts of matrix metalloproteinase degradation have no significant effect on cellular viability or proliferation (Lau, 2011). The stability of genipin-crosslinked tissues is superior to glutaraldehyde-crosslinked tissues when assessed over a 6 month period, stored in sterile water (Sung, 2001).

The anti-inflammatory properties of genipin were investigated by Chang et al. (2002). Bovine pericardium samples were decellularized and fixed with either glutaraldehyde or genipin. The samples fixed with genipin displayed a markedly reduced inflammatory response and tissue regeneration within 4 weeks, compared to those that were crosslinked with glutaraldehyde, which displayed persistent inflammation and no tissue regeneration at 26 weeks.

Genipin has been shown to rapidly inhibit uncoupling protein 2 (UCP2)-mediated proton leak in isolated pancreatic islets, increasing mitochondrial membrane potential, ATP production, and dose-dependently increasing insulin secretion (Zhang, 2006A). In the same study, genipin reversed high glucose- and obesity-induced $\beta$-cell dysfunction. 


\subsubsection{Other Crosslinking Methods}

Isocyanates, specifically hexamethylene diisocyanate, have been used to crosslink $\varepsilon$-amino groups of lysine in collagenous tissues, raising the degradation temperature by $20^{\circ} \mathrm{C}$ in a linear manner proportional to the decrease in free amine groups (Damink, 1995).

Acyl azides, including hydrazine and diphenylphosphoryl azide, react with carboxyl groups and $\varepsilon$-amino groups providing the same degree of crosslinking observed in glutaraldehyde systems with reduced cytotoxicity (Petite, 1995).

D,L-Glyceraldehyde has been used to replace glutaraldehyde, as it is nontoxic, and a natural product of metabolism. The exact mechanism of reaction between glyceraldehyde and amine groups within collagen and gelatin is still widely debated (Sisson, 2009).

\subsection{Alternatives to Primary Islets}

Despite advances in microenvironment restoration, a major issue associated with the clinical application of implanted islets, both immunosuppressed and immunoisolated, is the large shortfall between donated tissue supply and patient demand, especially as many isolation and implantation techniques require more than one donor pancreas per patient in order to restore normoglycemia (Robitaille, 2003). With current techniques, it has been estimated that the supply of donor pancreas tissue presently available would only provide islets for approximately $0.5 \%$ of the potential recipients (Lechner, 2003).

One method that has been shown to more efficiently use the islet cell mass is islet reaggregates, which are smaller and more consistent in size, don't suffer from central necrosis, and perform better in low-oxygen environments (O'Sullivan, 2010). This approach, currently applied to dispersed primary islets, could also be used with other alternative cell lines. 
It has been shown that embryonic stem (ES) cells can be induced to form islet-like clusters, however this research has been greatly restricted by ethical considerations (Lumelsky, 2001; Segev, 2004; Wang, 2009). Insulin-production has also been induced in progenitor cells isolated from the liver (Zalzman, 2003; Sapir, 2005), central nervous system (Hori, 2005), and spleen (Kodama, 2003), as well as bone-marrow derived mesenchymal stem cells (BM-MSC) and primitive stromal cells from umbilical-cord Wharton's jelly (UC-PSC) shown in Figure 15 (Wu, 2009).
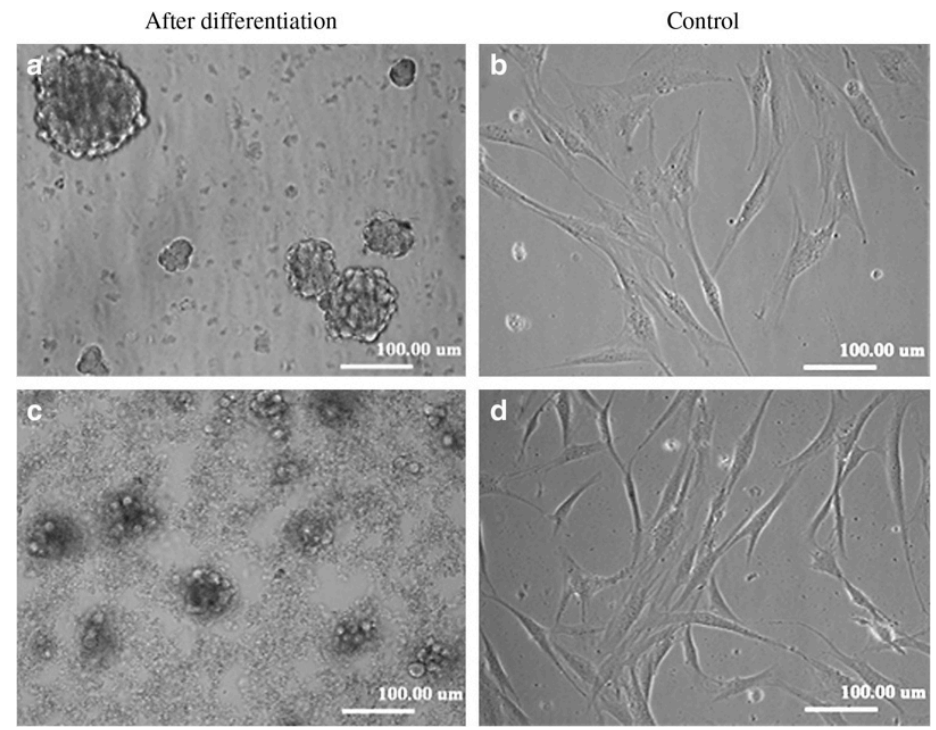

Figure 15. Formation of pancreatic islet-like clusters after UC-PSC (a, b) and BM-MSC (c, d) incubation in differentiation medium ( $\mathrm{Wu}, 2009)$.

Primitive stromal cells isolated from umbilical cord Wharton's jelly are a source of cells for differentiation into insulin-producing islet-like cell clusters. Isolation from umbilical cords is noninvasive, non-traumatic, and provides a large and widely available source of donor tissue, as the cord is traditionally disposed of as medical waste $(\mathrm{Wu}, 2009)$. Wu et al. (2009) induced pancreatic differentiation in UC-PSC which was detected after 3 days by immunocytochemical analysis for C-peptide, glucagon, and PDX-1, and a radioimmunoassay for insulin. Pancreatic and 
duodenal homeobox 1 (PDX-1) also known as insulin promoter factor 1 , is a commonly used marker of islet differentiation as it is a transcription factor for pancreatic development and $\beta$-cell maturation (Zhou, 2008).

Bara and Sambanis (2009) took a different approach to developing a tissue replacement for cadaver-derived islets, by engineering enteroendocrine L-cells to produce insulin and glucagonlike peptide. Enteroendocrine L-cells were chosen as they have the ability to secrete hormones directly into the bloodstream in a tightly controlled fashion, and process proinsulin into insulin and C-peptide. Immortalized murine GLUTag L-cells were transfected with a plasmid coexpressing human insulin and neomycin resistance to engineer the stable cell line, GLUTag-INS (Bara, 2008).

An immortalized human $\beta$-cell line MIN6 is often used in pancreas engineering studies as it is more resilient than the primary cell line, retains glucose-induced insulin secretion, and can be expanded, avoiding the large number of time-consuming and expensive rat islet isolations (Miyazaki 1990). However, the beneficial properties of MIN6 cells for cell culture also make them a poor islet analogue for viability studies, as they artificially inflate the efficacy and cytocompatibility of a process. For example, in a study on emulsion encapsulation, a $90 \pm 2 \%$ viability was reported after encapsulation for MIN6 cells, however, when primary cells were used from islet-depleted human pancreatic tissue, the viability was reduced to $71 \pm 4 \%$ (Hoesli, 2011). As MIN6 is an immortalized cell line, any in vivo study would first require long-term oncogenic studies. Additionally, the rat islet tumor cell line RIN-m and its secondary clone RIN-5F are also used in some studies (Kawazoe, 2009).

In order to meet the needs of future treatments, a new islet-like cell source will be required to replace the insufficient supply of cadaveric-islets. Many of the recombinant techniques and 
differentiation protocols, like that of UC-PSC, show real promise, but full implementation will require extensive in vivo study into the oncogenicity and stability of the islet-like cells produced.

\subsection{Summary}

It is clear that the Edmonton protocol islet transplantation with immunosuppression approach, while improving, is not an ideal solution, as the majority of the side-effects from islet transplants are due to immunosuppression, and not the procedure itself (Shapiro, 2000). The growing clinical database of immunoisolated islet transplant trials will lead to an optimization of the technique, providing results are appropriately stated with the requisite level of detail, outlined by de Vos et al. (2009A). One issue with many of the studies is the use of 2D culture as a control. It is well documented that $2 \mathrm{D}$ culture is detrimental to islet function, and is likely therefore used to artificially inflate the significance of incremental advancements to 3D culture. A transition to an encapsulated alginate microparticle control provides a more appropriate comparison for novel advances in 3D immunoisolation matrices while retaining a simple and well-studied control.

While advances are rightfully compared to the current state of the art in the tissue engineering solution to diabetes management, true success must always be viewed relative to current exogenous insulin therapy, which is minimally invasive, well understood, and has a proven history of safety and reliability. In addition, considerable work into insulin delivery and sustained release will only improve the efficacy and reduce the invasiveness of the treatment. Only once endocrine tissue engineering advances to a state where the benefits of the procedure outweigh those of insulin therapy, can it responsibly be adopted as the standard course of treatment. 


\section{Chapter 3}

\section{Research Objectives}

One of the primary challenges in islet tissue engineering is anoikis induced apoptosis and functional deficiencies. This is due primarily to the absence of integrin-mediated connections between islets and their environment as a result of enzymatic isolation and purification. By restoring the environmental conditions with matrices and ECM components, physiological islet function may one day be maintained for a significant period of time.

There currently exists a large pool of data in the fields of islet culture on fibrous matrices, ECMsupplemented matrices, and islet and matrix immunoisolation. To date, large cellular-aggregate seeding limitations have hindered the confluence of these three fields in a microcapsule geometry. These limitations can be overcome by a novel approach to scaffold production, seeding, and encapsulation wherein all steps are combined into a single process, weaving a fibrous scaffold around the cellular aggregate during particle formation. This approach eliminates the need for large pore geometries, allowing the scaffolds to be of sufficiently small size to be immunoisolated in an alginate microparticle while maintaining appropriate diffusion pathlengths, increasing the surface area for cell-scaffold contact, and providing a modular platform for a future investigation. In order to achieve the envisioned system, the principal objectives of this study were to:

1. Develop and characterise a method to produce and covalently crosslink discrete protein fibers on a scale that would allow for the encapsulation of a fibrous network within a $<$ $300 \mu \mathrm{m}$ microparticle, while avoiding processes with cytotoxic residual components and metabolites. 
2. Produce $<300 \mu \mathrm{m}$ ionically-gelled alginate microparticles containing a crosslinked protein fiber network, aiming for consistency and reproducibility.

3. Incorporate primary islets extracted from rats into the particles and measure the effect on islet viability, cohesion, apoptosis, and insulin secretion in vitro, relative to controls, over a period of 21 days. 


\section{Chapter 4}

\section{Materials and Methods}

\subsection{Materials}

Pharmaceutical-grade Protanal LF 10/60 sodium alginate was purchased from FMC BioPolymers (Drammen, Norway). Genipin was purchased from Challenge Bioproducts Co., Ltd. (Taiwan). Activa-TI ${ }^{\mathrm{TM}}$ (Ajinomoto), a $1 \%$ microbial transglutaminase (mTG) culinary preparation, was kindly donated by Thomas, Large \& Singer Inc. (Montreal, Canada). Analytical grade sodium alginate, gelatin (type A, 300 Bloom, porcine skin) and glucose-free RPMI-1640 were purchased from Sigma-Aldrich Canada Ltd. (Oakville, Canada). Polysucrose 400 (Ficoll), glucose (EuroCollins) solution, and electrolyte additive solution were purchased from Mediatech Inc. (Herndon, USA). Rat insulin ELISA kits were purchased from Mercodia (Winston Salem, USA). CellTiter $96^{\circledR}$ Aqueous One Solution Cell Proliferation Assay from Promega Corp (Madison, USA) was used to quantify metabolic activity. A Quant-iT ${ }^{\mathrm{TM}}$ PicoGreen $^{\circledR}$ dsDNA kit from Molecular Probes ${ }^{\circledR}$ (Burlington, Canada) was utilized to quantify double stranded DNA (Appendix A). Unless otherwise specified, alginate purification reagents were purchased from Fisher Scientific. All other materials were of analytical grade and purchased from Sigma-Aldrich Canada Ltd (Oakville, Canada). 


\subsection{Methods}

\subsubsection{Electrospun Gelatin Nanofibers}

Nanofibers were electrospun from a 10\% gelatin (type A, 300 Bloom) solution dissolved in 50\% glacial acetic acid, 30\% ethyl acetate, and 20\% distilled water (Song, 2008; Sisson, 2009). Access to the electrospinning apparatus with programmable mandrel was kindly provided by Dr. Kimberly Woodhouse, Queen's University. The gelatin solution was extruded at $1 \mathrm{~mL} / \mathrm{h}$ through a $20 \mathrm{G}$ blunt needle with a $+12 \mathrm{kV}$ potential. The mandrel was placed $10 \mathrm{~cm}$ from the tip of the needle and wrapped in silica-treated non-stick aluminum foil (Alcan). Collection proceeded for 30 minutes under both random mat $(\omega=0 \mathrm{rpm})$ and aligned $(\omega=100 \mathrm{rpm})$ conditions. Neither test was performed with translational motion of the mandrel. Upon completion, the fiber-coated aluminum foil was cut from the mandrel and dried under vacuum.

A number of crosslinking techniques were attempted for the nanofiber system. The first involved suspending the nanofibrous mat on the surface of a $4^{\circ} \mathrm{C}$ solution of $1 \%$ Activa-TI microbial transglutaminase in distilled water, previously dissolved at $45^{\circ} \mathrm{C}$ in $50 \mathrm{mM} \mathrm{pH} 6.02-(\mathrm{N}-$ morpholino)ethanesulfonic acid hydrate (MES) buffer. The system was allowed to passively return to room temperature overnight, at which point it was heated to $37^{\circ} \mathrm{C}$ for $1 \mathrm{~h}$ to complete crosslinking.

The second crosslinking approach was a non-aqueous system in which a nanofibrous mat was suspended within a small vacuum desiccator produced from a $20 \mathrm{~mL}$ scintillation vial, over a 5\% genipin solution in $100 \%$ ethanol (Figure 16). House vacuum was used to evacuate the chamber and the system was heated to $45^{\circ} \mathrm{C}$ with a waterbath. 


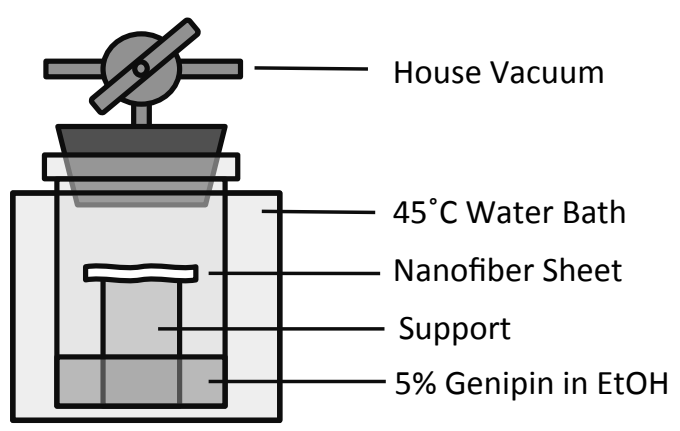

Figure 16. Genipin/Ethanol vapour crosslinking desiccator.

\subsubsection{Drawn Gelatin Microfibers}

Fibers were produced by an alginate-mediated extrusion method adapted from Yang et al. (2009). In the initial system, a solution of $0-2 \%$ gelatin (Type A, 300 Bloom) and $1.5 \%$ sodium alginate (Sigma Aldrich) in distilled water was extruded from a $18-30 \mathrm{G}$ syringe into a bath of $2 \% \mathrm{CaCl}_{2}$ (Figure 17). The extruded fiber was drawn out of the bath and around a $60 \mathrm{~mm}$ diameter mandrel attached to a variable speed Caframo Type RZR1-64 stirrer.

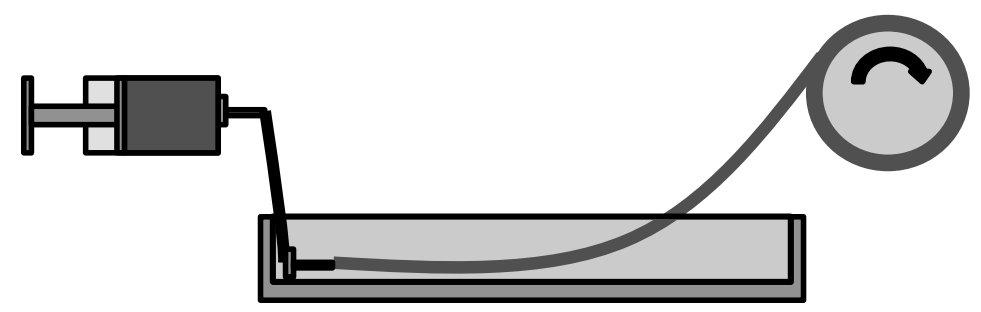

Figure 17. Initial microfiber spinning system with drawing bath and mandrel.

Through a series of optimizations, a wet-drawn vortex system was developed. A $45^{\circ} \mathrm{C}$ solution of $1.5 \%$ sodium alginate (Sigma Aldrich) and 1.5\% gelatin (Type A, 300 Bloom) in distilled water was filtered $(0.22 \mu \mathrm{m})$, and degassed under vacuum. The solution was loaded into a syringe and extruded through a $35 \mathrm{G}$ blunt tipped needle (ID $80 \mu \mathrm{m}$ ) via flexible silicone tubing at a rate of $2.4 \mathrm{~mL} / \mathrm{h}$ using a syringe pump. The syringe was heated by a thermal gel pack heated to $70^{\circ} \mathrm{C}$ in a 
water bath, and replaced every 20 minutes. The tip of the needle was submerged at a $45^{\circ}$ angle into a $45^{\circ} \mathrm{C}$ vortex of sterile filtered $(0.22 \mu \mathrm{m}) 1.5 \%$ gelatin and $2 \% \mathrm{CaCl}_{2}$ dissolved in $450 \mathrm{~mL}$ of distilled water (Figure 18). The vortex was formed by a $50 \mathrm{~mm}$ magnetic stir bar rotating at 700 rpm within a $600 \mathrm{~mL}$ beaker $(12 \mathrm{~cm} \times 8.5 \mathrm{~cm}$ diameter). The vortex system was maintained at $45^{\circ} \mathrm{C}$ by immersing it in a temperature-controlled waterbath.

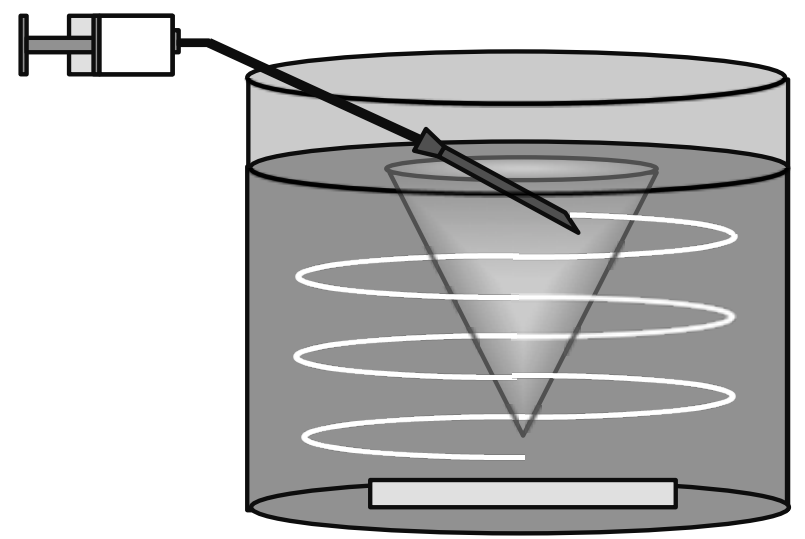

Figure 18. Extrusion of gelatin/alginate solution into a vortex of gelatin and $\mathrm{CaCl}_{2}$

Gelatin-alginate fibers were transferred to a protein-crosslinking genipin solution of $50 \mathrm{mM}$ $\mathrm{CaCl}_{2}$ and $100 \mathrm{mM}$ TRIS which was adjusted to $\mathrm{pH} 8.0$ with $1 \mathrm{M} \mathrm{NaOH}$ before the addition of $0.25 \mathrm{wt} \%$ genipin. To aid with solubility, genipin was initially dissolved in $95 \%$ ethanol at 0.10 $\mathrm{g} / \mathrm{mL}$ and an aliquot was added to the buffered $\mathrm{CaCl}_{2}$. Fibers were allowed to crosslink for $48 \mathrm{~h}$ at $25^{\circ} \mathrm{C}$ in $50 \mathrm{~mL}$ tubes on a RotoShake Genie ${ }^{\circledR}$ rotating end-over-end at $4.5 \mathrm{rpm}$. The alginate support matrix and uncrosslinked gelatin were removed from the fibers by chelating in a $37^{\circ} \mathrm{C}$ solution of $55 \mathrm{mM}$ sodium citrate, $30 \mathrm{mM}$ EDTA, and $0.15 \mathrm{M} \mathrm{NaCl}$ with agitation, changing the chelation solution three times. The gelatin fibers were then dried and sterilized in a sequential ethanol bath $(50 \%, 75 \%, 95 \%)$ followed by vacuum drying. 
When required for encapsulation, the fibers were rehydrated in sterile $\mathrm{pH} 7.4$ phosphate buffered saline (Invitrogen 70011-044) and dissociated into discrete $200 \mu \mathrm{m}$ fibers with an ultrasonic homogenizer for 5 minutes (Cole-Parmer 4710-CV17, 60\% duty, output 4). The sonicated suspension of fibers in PBS was allowed to settle for 10 minutes before the unsettled fraction was collected and pelleted at $2000 \mathrm{x} \mathrm{g}$ for 10 minutes.

Fiber diameters and lengths were sized from representative light microscopy images with $n=300$ and 200 respectively.

\subsubsection{Emulsion Encapsulation}

Early acellular studies utilized a modified internal gelation emulsion encapsulation (Poncelet $1992,1995)$. The aqueous phase contained $4 \%$ sodium alginate, $0.3 \%$ ultrafine calcium carbonate (Specialty Minerals Inc, USA) and 50 vol \% hydrated gelatin fibers (concentrated at $2000 \mathrm{~g} \mathrm{x} 10$ $\min )$. The alginate mixture $(30 \mathrm{~mL})$ was dispersed 1:2 in $60 \mathrm{~mL}$ sunflower oil with an impeller at $330 \mathrm{rpm}$. The specifications of the emulsion system are detailed in Figure 19. At five minutes, the system was acidified with $120 \mu \mathrm{L}$ of glacial acetic acid in $10 \mathrm{~mL}$ sunflower oil to solubilize the calcium carbonate and crosslink the alginate phase. After one minute of acidification, $50 \mathrm{mM}$ $\mathrm{CaCl}_{2}$ was added and the particles were allowed to settle. The oil phase was removed by aspiration and the particles were stored in $50 \mathrm{mM} \mathrm{CaCl}_{2}$. 

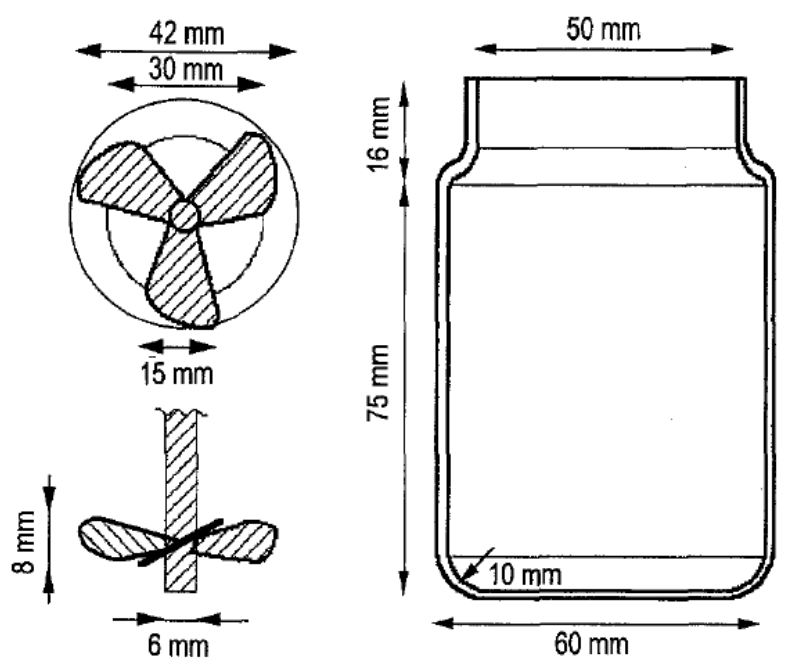

Figure 19. Dimensions of impeller and container used in the internally-gelled emulsion system (Poncelet, 1995)

\subsubsection{Fluorescent Labeling of Gelatin and Alginate}

For fluorescent imaging of islet-like particles gelatin was labeled with fluorescein isothiocyanate (FITC). FITC-gelatin was also used with rhodamine B isothiocyanate (RBITC) labeled alginate for confocal imaging of gelatin membranes (Appendix B). Fluorescent labeling protocols were modified from those developed by Mlaenovska et al. (2007) and Clausen et al. (2000).

FITC dissolved in dimethylsulphoxide (DMSO) at $2 \mathrm{mg} / \mathrm{mL}$ was slowly added to gelatin dissolved in $0.1 \mathrm{M} \mathrm{Na}_{2} \mathrm{CO}_{3}$. The solution was incubated at $4^{\circ} \mathrm{C}$ for 8 hours, supplemented with $\mathrm{NH}_{4} \mathrm{Cl}$ to a final concentration of $50 \mathrm{mM}$, then incubated at $4^{\circ} \mathrm{C}$ for an additional 2 hours. The gelatin was then loaded into dialysis tubing with a molecular weight cut off of $6,000-8,000$, and dialyzed against distilled water for 72 hours, changing the distilled water every 8 hours.

A solution of $2 \%$ alginate was prepared in distilled water and adjusted to $\mathrm{pH} 8.0$ with $1 \mathrm{M} \mathrm{NaOH}$. RBITC was added slowly from a $1 \mathrm{mg} / \mathrm{mL}$ aliquot prepared in DMSO. The solution was stirred 
for 1 hour at $40^{\circ} \mathrm{C}$ before the addition of $50 \mathrm{mM} \mathrm{NH}_{4} \mathrm{Cl}$ and dialysis, following the same protocol as the FITC-gelatin.

Following dialysis, the alginate and gelatin solutions were frozen in $20 \mathrm{~mL}$ aliquots at $-80^{\circ} \mathrm{C}$ and lyophilized.

\subsubsection{Endotoxin-Free Work}

All investigations using islets were performed under endotoxin-free conditions. When possible, disposable endotoxin-free materials were used. All labware was washed and soaked in Extran soap, rinsed three times with distilled water, once in $2 \mathrm{~N} \mathrm{HCl}$, an additional two times in distilled water, and finally with sterile water. Glass and metal labware were sent for steam sterilization at $200^{\circ} \mathrm{C}$ for 2 hours, while plastic was sterilized with ethylene oxide.

All solutions were prepared with endotoxin-free labware using sterile water. Once prepared, all solutions underwent additional sterile $(0.22 \mu \mathrm{m})$ filtration.

\subsubsection{Islet Isolation}

Islets were isolated from male Sprague Dawley rats (Charles River Institute, St.-Constant, Canada) according to established techniques (Gotoh, 1985; Langlois, 2009) and under the approval of the Maisonneuve-Rosemont Hospital Animal Ethics Committee.

Rats were anesthetized with isofluorane and their abdomens were shaved and disinfected. The abdominal cavity was exposed, cooled with Hank's balanced salt solution (HBSS) stored on ice, and the bile duct was located and clamped. Once the bile duct was cannulated via a small incision on the surface of the duct, the clamps were removed and the animal was sacrificed. When the blood had drained sufficiently, the duct was infused with $\sim 10 \mathrm{~mL}$ of $1 \mathrm{mg} \mathrm{mL}^{-1}$ collagenase $\mathrm{V}$ 
(Sigma Aldrich C9263; lot 128H8632) in HBSS stored on ice. The distended pancreas was then separated from the surrounding viscera and placed in a tube containing an additional $5 \mathrm{~mL}$ collagenase $\mathrm{V}$ solution on ice. The chilled tubes were warmed in a water bath and allowed to digest at $37^{\circ} \mathrm{C}$ for $30 \mathrm{~min}$. All purification work was performed in an ice-bath and $4^{\circ} \mathrm{C}$ centrifuge. The digest was washed repeatedly with HBSS and EuroCollins solution supplemented with 10\% and $2 \%$ newborn calf serum (NCS) respectively. Between each wash the cells were pelleted at $300 \mathrm{x}$ g. Following washing, islets were separated from the exocrine cell population by discontinuous EuroFicoll density gradient $(1.108,1.096,1.069,1.045 \mathrm{~g} / \mathrm{mL})$. The cell pellet was suspended in $15 \mathrm{~mL}$ of $1.108 \mathrm{~g} / \mathrm{mL}$ EuroFicoll within a $50 \mathrm{~mL}$ centrifuge tube. The three additional concentrations were carefully loaded in sequence in $10 \mathrm{~mL}$ volumes to create a stepwise concentration gradient (Figure 20).

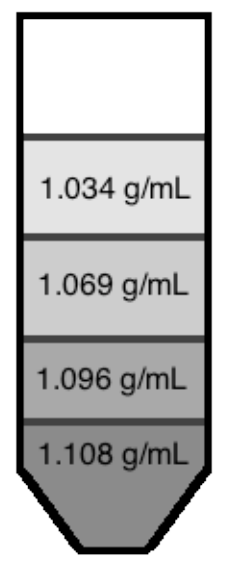

Figure 20. Discontinuous EuroFicoll gradient. Cell mass was loaded in the $1.108 \mathrm{mg} / \mathrm{mL}$ fraction and the purified islet fraction was collected at the $1.096-1.069 \mathrm{~g} / \mathrm{mL}$ boundary following centrifugation.

The tube was spun at $600 \mathrm{x}$ g for 10 minutes and allowed to decelerate without brake in order to maintain the precision of the stratified EuroFicoll layers. The islet-rich cell fraction at the 1.096 
$\mathrm{g} / \mathrm{mL}$ and $1.069 \mathrm{~g} / \mathrm{mL}$ boundary was collected, and washed with EuroCollins and HBSS supplemented with $2 \%$ NCS. Islets were then hand picked twice over an inverted-light microscope, providing both a positive and negative screening, and cultured overnight, evenly distributed on non-adhesive polystyrene culture dishes. The absence of islets in the remaining acinar and exocrine cell tissue pellet was confirmed by dithizone (DTZ) staining.

The DTZ solution is composed of $2.5 \mathrm{mg} / \mathrm{mL}$ diphenylthiocarbazone in DMSO diluted 1/10 in HBSS. A small aliquot of DTZ solution is added to a cell suspension to visualize islet tissue, as the interaction between DTZ and the high levels of zinc present in islet tissue lead to an easily identified red colouration (Latif, 1988).

All cultures were performed in $5.5 \mathrm{mM}$ glucose RPMI-1640 supplemented with $10 \%$ fetal bovine serum, $1 \%$ penicillin-streptomycin, $0.25 \%$ amphotericin $\mathrm{B}, 15 \mathrm{mM}$ HEPES, and $23 \mathrm{mM}$ sodium bicarbonate at $37^{\circ} \mathrm{C}$ and $5 \% \mathrm{CO}_{2}$.

\subsubsection{Alginate Purification}

Pharmaceutical-grade Protanal LF 10/60 sodium alginate was purified by a modified Klöck method (Langlois, 2009). Alginate was first purified by a 30 minute chloroform extraction and 30 minute drying period, repeated three times. The dry alginate was then dissolved in water and treated with an equal mass of activated carbon for 4 hours followed by neutral carbon for an additional 4 hours. The carbon was removed following each purification by centrifugation at $23,000 \mathrm{x}$ g for 1 hour, and finally by a $0.22 \mu \mathrm{m}$ Steripak filter (Millipore) and peristaltic pump. Beads were formed from the filtered alginate by extruding the solution into $50 \mathrm{mM} \mathrm{BaCl}_{2}$. The barium alginate beads were then extracted three times in $1 \mathrm{M}$ acetic acid for $14 \mathrm{~h}, 12 \mathrm{~h}$, and $16 \mathrm{~h}$; $500 \mathrm{mM}$ sodium citrate three times for $8 \mathrm{~h}, 10 \mathrm{~h}$, and $8 \mathrm{~h} ; 50 \%$ ethanol supplemented with 5\% 
acetone for $16 \mathrm{~h}$; and $70 \%$ ethanol supplemented with $5 \%$ acetone for $16 \mathrm{~h}$, with a wash in sterile water following every extraction. The beads were then washed with $20 \mathrm{mM} \mathrm{BaCl}$ and sterile water before being transferred to an equal volume of $250 \mathrm{mM}$ EDTA solution for dissolution overnight. The chelated alginate solution was then filtered through a $0.22 \mu \mathrm{m}$ Steritop filter (Millipore) and transferred to dialysis membrane. The alginate was dialyzed against $150 \mathrm{mM}$ $\mathrm{NaCl}$ three times for $5 \mathrm{~h}, 6 \mathrm{~h}$, and $10 \mathrm{~h}$, followed by sterile water for $5 \mathrm{~h}, 6 \mathrm{~h}$, and $10 \mathrm{~h}$. The dialyzed alginate was supplemented with $\mathrm{NaCl}$ to a final concentration of $10 \mathrm{mM}$, filtered with a $0.22 \mu \mathrm{m}$ Steritop, and precipitated by the addition of 2 volumes of $100 \%$ ethanol with stirring for 30 min. The alginate precipitate was then recovered by centrifugation, frozen at $-80^{\circ} \mathrm{C}$ and lyophilized. The final product was stored within $50 \mathrm{~mL}$ conical tubes at $4^{\circ} \mathrm{C}$. All solutions used in the purification were prepared with sterile water under endotoxin-free conditions and $0.22 \mu \mathrm{m}$ filtered before use.

\subsubsection{Electrostatic-Mediated Dropwise Encapsulation}

Fiber and islet co-encapsulation proceeded by electrostatic-mediated dropwise particle formation. The extruded solution contained 40 vol $\%$ hydrated fiber, 60 vol $\% 1.6 \%$ purified sodium alginate dissolved in $0.9 \%$ saline, and 2,000 islets $/ \mathrm{mL}$. Islet concentration of $2,000-10,000$ islets $/ \mathrm{mL}$ are used in the literature (Mallet, 2009; Jourdan, 2011), a lower concentration was selected to reduce the prevalence of multiple islets per capsule, which was observed in preliminary studies at 5,000 islets $/ \mathrm{mL}$. An alginate concentration of $1.6 \%$ was selected as it is a commonly implemented intermediate concentration (Calafiore, 2006A). Alginate concentrations used in the literature ranges from $0.6 \%$ to $2.3 \%$ (Lim, 1980; Langlois, 2009). Hydrated fibers were concentrated at $2000 \mathrm{x}$ g for $10 \mathrm{~min}$, dispensed with a MICROMAN ${ }^{\circledR}$ M1000 (Gilson Inc.) positive displacement 
pipette, and homogenously distributed within the alginate by repeated pipetting. Islets were washed with serum-free HBSS, pelleted at $300 \mathrm{x}$ g for 2 minutes, and suspended in the fiberladen alginate by repeated pipetting with an M1000 positive displacement pipette.

The alginate suspension was drawn into a length of endotoxin-free tubing pre-filled with pure alginate and sufficiently long to hold the entire sample. As the sample was drawn into the tubing, the pure alginate was drawn into the attached syringe. The sample was separated from the pure alginate by an air gap to allow for easy tracking. The syringe was then placed in a syringe pump and a $22 \mathrm{G}$ HPLC needle was affixed to the end of the tubing. The fiber-laden solution was extruded at $2 \mathrm{~mL} / \mathrm{h}$ while the less-viscous fiber-free control solution was extruded at $5 \mathrm{~mL} / \mathrm{h}$ to ensure consistent final particle diameters. The alginate solution was extruded into $90 \mathrm{~mL}$ of unmixed $10 \mathrm{mM} \mathrm{BaCl} 2$ buffered at $\mathrm{pH} 7.4$ with $12.5 \mathrm{mM}$ HEPES within a plastic beaker containing a strip of metallic tape.

The HPLC needle was charged with $+11 \mathrm{kV}$ of potential for $2.5 \mathrm{~ms}$ every $25 \mathrm{~ms}$ via a clamp affixed near the tip and connected to a custom-made high voltage pulsed power supply. The collection bath was grounded through the same device via a clamp on the metallic tape affixed to the inside of the container. The distance between the tip of the needle and the surface of the collection bath was approximately $10 \mathrm{~cm}$.

Upon completion of the extrusion the particles were allowed to settle, the $\mathrm{BaCl}_{2}$ was aspirated, and the particles were washed with complete media before culturing in non-adhesive polystyrene dishes. 


\subsubsection{Particle Culture and Assessment}

Islet-laden particles were incubated in sterile non-adhesive polystyrene dishes for 1, 7, 14 and 21 days at $5 \% \mathrm{CO}_{2}$ and $37^{\circ} \mathrm{C}$.

\section{Viability}

Viability was assessed with propidium iodide (PI) - acridine orange (AO) staining of 100 particles for each condition. Stock solutions of $1 \mathrm{mg} / \mathrm{mL}$ PI and $0.01 \mathrm{mg} / \mathrm{mL} \mathrm{AO}$ were prepared in sterile phosphate buffered saline (PBS) and stored at $-20^{\circ} \mathrm{C}$. A staining solution of $500 \mu \mathrm{L}$ PI stock and $250 \mu \mathrm{L}$ AO stock was prepared in sterile PBS. Particles were allowed to settle, the media was aspirated, replaced with staining solution, and incubated for 10 minutes at room temperature. The staining solution was then aspirated and the particles were suspended in a small volume of PBS. The particles were viewed under a fluorescent microscope with a blue excitation filter (490 nm). All work with PI and AO was performed under low-light conditions.

Under fluorescent microscopy, viable cells stain green and dead cells stain red. AO-PI staining is a measure of membrane integrity. AO readily enters living cells staining them green, while PI, which can only penetrate dead cells, binds to nucleic acids and fluoresces red (Bank, 1987).

\section{Metabolic Activity}

Metabolic activity was measured with a CellTiter $96^{\circledR}$ Aqueous One Solution Cell Proliferation Assay (Promega Corp) on 100 particles in triplicate. Particles were placed in a 96 well plate with $100 \mu \mathrm{L}$ of culture medium and $20 \mu \mathrm{L}$ of reagent. At 4 hours $50 \mu \mathrm{L}$ of each well was transferred to an adjacent empty well to be read in a plate reader at $490 \mathrm{~nm}$. The transfer was performed in order to avoid measuring the absorbance of the particles covering the bottom of the well. After 
reading, the $50 \mu \mathrm{L}$ was replaced and incubated for an additional 20 hours, at which time $50 \mu \mathrm{L}$ was removed and analyzed at $490 \mathrm{~nm}$ in the same manner as before.

The colour change at $490 \mathrm{~nm}$ is due to the reduction of [3-(4,5-dimethylthiazol-2-yl)-5-(3carboxymethoxyphenyl)-2-(4-sulfophenyl)-2H-tetrazolium (MTS) by NADPH or NADH produced by dehydrogenase enzymes in metabolically active cells into a coloured formazan salt (Berridge, 1993).

\section{Glucose-Induced Insulin Secretion}

Insulin secretion was determined by rat-insulin enzyme-linked immunosorbent assay (ELISA) (Mercodia) performed on aliquots of media collected following $1 \mathrm{~h}$ incubations of 50 microparticles in triplicate in a 24 well plate. The assay was calibrated with $0.15-5.5 \mu \mathrm{g} / \mathrm{L}$ rat insulin provided with the kit. The same particles were used at the same conditions for all time points. Culture media was removed from the wells by pipette over an inverted light microscope and discarded. Wells were washed with $200 \mu \mathrm{L}$ of $3.3 \mathrm{mM}$ glucose serum-free medium, which was removed by pipette. All wells were then filled with $500 \mu \mathrm{L}$ of $3.3 \mathrm{mM}$ glucose serum-free medium and incubated for 1 hour at $5 \% \mathrm{CO}_{2}$ and $37^{\circ} \mathrm{C}$. Upon completion, media was removed from the wells by pipette over an inverted light microscope and stored in $200 \mu \mathrm{L}$ aliquots at $80^{\circ} \mathrm{C}$. The wells were then filled with either $3.3,5.5,11$, or $16.5 \mathrm{mM}$ glucose serum-free medium and incubated for 1 hour at $5 \% \mathrm{CO}_{2}$ and $37^{\circ} \mathrm{C}$. Upon completion, media was again removed from the wells by pipette over an inverted light microscope and stored in $200 \mu \mathrm{L}$ aliquots at $-80^{\circ} \mathrm{C}$. Wells were then refilled with complete $5.5 \mathrm{mM}$ glucose medium and incubated until the next time point.

Upon completion of all time points, the aliquots were thawed and analyzed undiluted by ratinsulin ELISA. ELISA works based on the binding of antibodies to very specific recognition sites 
on the insulin molecule. The Mercodia system uses the 'sandwich technique' where the insulin in solution reacts with an anti-insulin antibody fixed to the polystyrene well while a second antiinsulin antibody is introduced to react with the bound insulin at a different recognition site. The second antibody is conjugated with a horseradish peroxidase which cleaves the substrate 3,3',5,5'-tetramethylbenzidine (TMB) to produce a soluble blue product. The reaction is stopped with the addition of sulfuric acid which changes the colouration from blue to yellow $(450 \mathrm{~nm})$. The absorbance of the solution at $450 \mathrm{~nm}$ is proportional to the concentration of insulin in the media (Mercodia, 2010). The use of bound antibodies allows the wells to be washed, removing all components of the media other than insulin, preventing cross-reactions and interference.

\section{Apoptosis}

Apoptosis was determined from nuclear morphology visualized by Hoechst 33258 (Molecular Probes Inc.) fluorescent nuclear staining. Fifty particles for each condition were collected at each time point and stored in $10 \%$ buffered formalin. Upon completion of the study, the formalin was aspirated and the particles were embedded in $50 \mu \mathrm{L}$ of $2 \%$ low-melting point agarose dissolved in PBS at $37^{\circ} \mathrm{C}$, and placed on ice to solidify the agarose pellet. Pellets were then placed in cuvettes and dehydrated in a sequential ethanol bath. Samples were then taken to John daCosta, Department of Pathology and Molecular Medicine, Queen's University for paraffin embedding and sectioning.

Slides were deparaffinized and rehydrated in a 9-step sequential xylene-ethanol-distilled water wash. The rehydrated slides were stained with a drop of $62.5 \mu \mathrm{g} / \mathrm{mL}$ Hoechst in buffered formalin for 2 minutes, washed with distilled water, mounted with a drop of Fluorescence Mounting Medium (Dako Canada Inc.), sealed with a coverslip and nail polish, and stored at $4^{\circ} \mathrm{C}$ in the dark. 
Nuclear morphology was observed under cyan excitation on a fluorescent microscope. Spherical nuclei were labeled viable, while compact aggregated nuclei were labeled apoptotic.

\section{Fiber Integrity and Crosslinking}

Fiber integrity and crosslinking efficiency were assessed by ninhydrin primary amine assay and bicinchoninic assay (BCA).

The ninhydrin assay was performed to measure the concentration of free primary amines in genipin-crosslinked fibers relative to uncrosslinked fibers. Dehydrated samples were placed in 2 $\mathrm{mL}$ of $0.1 \mathrm{M} \mathrm{pH} 5$ phthalate buffer in distilled water. All vials were heated in a water bath at $85^{\circ} \mathrm{C}$ for 5 minutes then cooled to room temperature. Once cooled, $1 \mathrm{~mL}$ of $2 \%$ ninhydrin in DMSO was added to each vial, and the vials were placed in a boiling water bath for 10 minutes. The samples were cooled for 10 minutes, and $5 \mathrm{~mL}$ of methanol was added. The absorbance at 570

$\mathrm{nm}$ was measured by spectrophotometer. The extent of crosslinking was determined from equation 2 .

$$
\% \text { Crosslinked }=1-\frac{\text { Absorbance of crosslinked samples-Average blank }}{\text { Absorbance of uncrosslinked samples-Average blank }}
$$

Fiber integrity was measured by a 70-day incubation and soluble protein quantification by micro bicinchoninic (micro BCA) assay (Thermo Scientific). Crosslinked dehydrated gelatin fiber samples were placed in sealed vials containing $1.5 \mathrm{~mL}$ of sterile PBS and incubated at $37^{\circ} \mathrm{C}$. PBS was selected to mimic the osmolarity of in vitro culture conditions. Additionally, salt solutions aid in the dissociation of proteins by masking the electrostatic charge of the polymers, thereby minimizing the effects of un-crosslinked gelatin adsorption (Ménard, 2010). The supernatant was collected on days 1, 3, 6 and 70 following fiber pelleting at 10,000 x g for 2 minutes and stored at $-80^{\circ} \mathrm{C}$. Equal $150 \mu \mathrm{L}$ volumes of thawed supernatant and micro BCA working reagent were 
combined in a 96 well plate and incubated at $37^{\circ} \mathrm{C}$ for 2 hours. The presence of protein was detected by increased absorbance at $562 \mathrm{~nm}$. A calibration ladder of $0.5-40 \mu \mathrm{g} / \mathrm{mL}$ uncrosslinked gelatin in PBS was analyzed in the same manner.

\section{Electron Microscopy}

Samples were vacuum-dried, fixed to aluminum stubs with carbon tape, gold sputtered, and imaged on a JEOL 840 (USA) scanning electron microscope with an accelerating voltage of $10 \mathrm{kV}$.

\section{Statistical Analysis}

All values are stated \pm standard error for replicate data sets and all error bars on plots are also indicating standard error about the mean, unless otherwise stated. All statements of significance are reported for 2 -sided student's t-tests at the $95 \%$ confidence level, unless otherwise stated. 


\section{Chapter 5}

\section{Results and Discussion}

The investigation started with a single goal, to bring together developments in islet culture systems in a manner that had previously been limited by the practicalities of geometry. The importance of immunoisolation was paramount, as the side effects of immunosuppression are well documented (Shapiro, 2000) and unjustified for a diabetes therapy. As alginate encapsulation is a well-studied system, it seemed the logical foundation and control system for the development of a modified islet encapsulation system.

Building off a background of aforementioned studies on the growth of islets within macroscopic fibrous matrices and protein-embedded gels, the goal became the insertion of a protein matrix specifically tailored to the limitations of islet and microparticle dimensions. The approach, which

is currently unpublished in the literature, was to form a matrix of protein fibers around an islet during the encapsulation process, eliminating the need for islet seeding geometries and allowing for greatly enhanced contact, increasing the surface area for islet-ECM integrin-mediated connections.

The research program can be divided into three main studies: protein fiber production, fiber-laden particle production, and a preliminary in vitro investigation. 


\subsection{Protein Fiber Production}

\subsubsection{Selection of Gelatin}

Gelatin was selected for the production of fibers due to its previous use in matrices (CultiSphereS) and fibers (Yang, 2009), high availability of unhindered primary amines relative to collagen, ease of solubility under a wide range of conditions in its uncrosslinked state, and low cost. 300 Bloom gelatin was specifically chosen for its higher molecular weight relative to low Bloom gelatins, resulting in superior mechanical properties.

\subsubsection{Electrospun Gelatin Nanofiber Investigation}

Discrete gelatin nanofibers were electrospun from a $10 \%$ gelatin solution, producing an average fiber diameter of $251 \pm 20 \mathrm{~nm}(\mathrm{n}=55)$ (Figure 21).

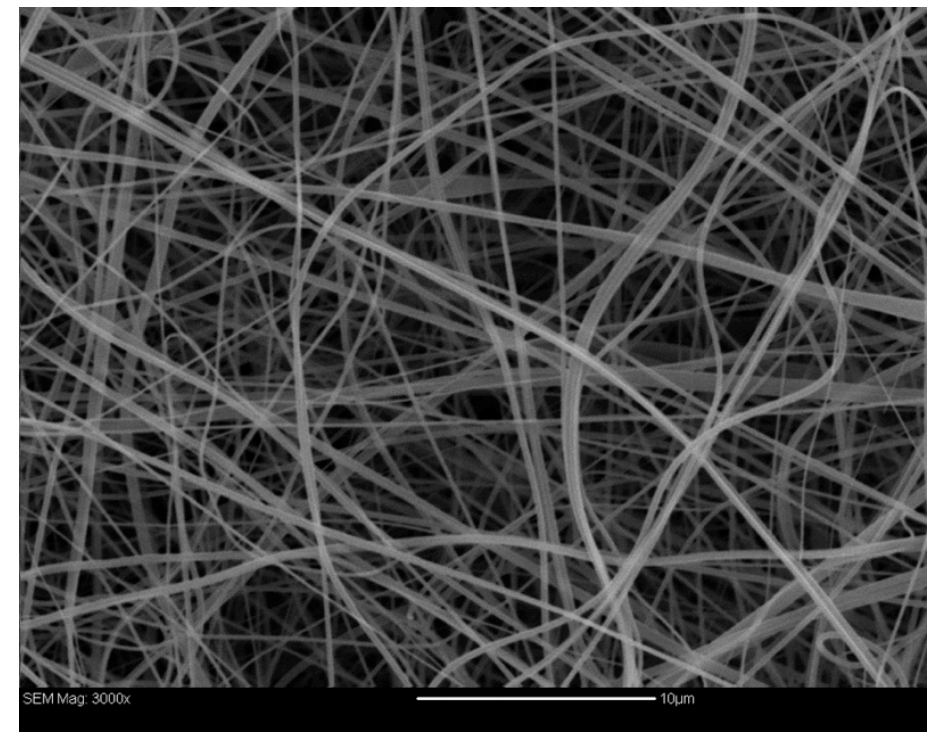

Figure 21. SEM image of randomly oriented electrospun gelatin nanofibers (scale bar represents $10 \mu \mathrm{m})$. 
As the fibers were spun from an aqueous ethyl acetate-acetic acid-water system, they were readily soluble in aqueous solutions at room temperature. For this reason, attempts to crosslink the fibers with genipin and transglutaminase solutions were ultimately unsuccessful at producing discrete gelatin fibers. In the presence of the aqueous crosslinking system, the fibers constricted axially, shrinking the dimensions of the fiber mat, while swelling radially and partially dissolving before the crosslinking agent attained sufficient integrity (Figure 22). The result was a fused mat of fibers with reduced dimensions but conserved geometry.

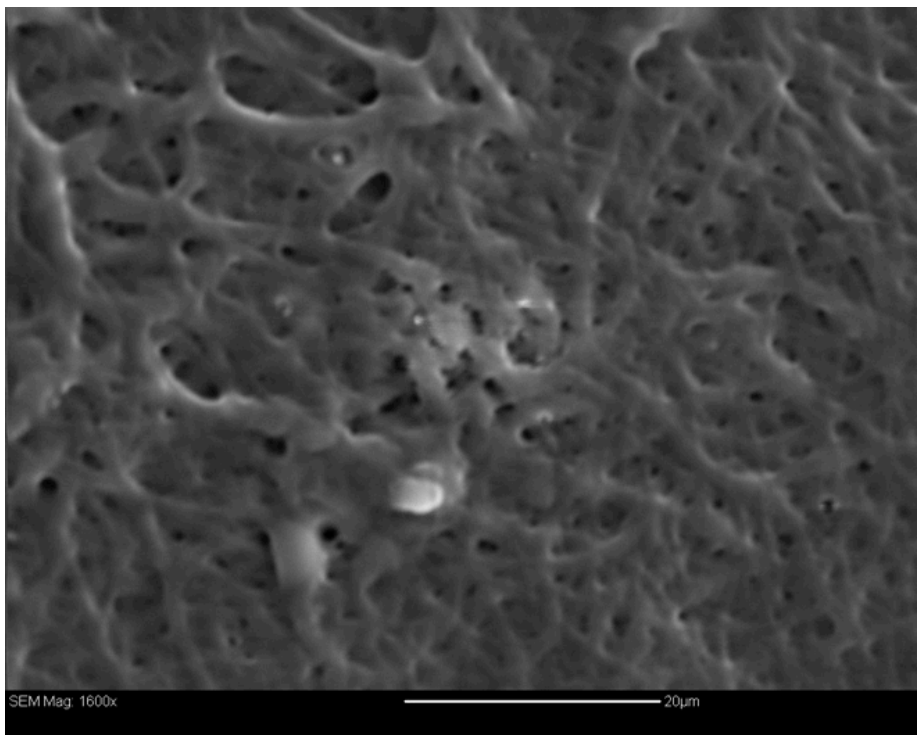

Figure 22. SEM image of genipin-crosslinked gelatin nanofiber sheet (scale bar represents $20 \mu \mathrm{m})$.

In the transglutaminase crosslinking system, kinetics are strongly influenced by temperature, resulting in negligible crosslinking before complete nanofiber dissolution at optimal conditions for transglutaminase $\left(\mathrm{pH} 6.0,37^{\circ} \mathrm{C}\right)$. Electrospun mats were then floated on the surface of a $4^{\circ} \mathrm{C}$ transglutaminase solution, which was allowed to passively rise to room temperature. This method produced crosslinked sheets of gelatin, but was unable to overcome the interplay between dissolution and crosslinking kinetics. As gelatin is insoluble in ethanol, crosslinking in various 
ethanol solutions was attempted. However, the Activa transglutaminase product was also insoluble in ethanol, and crosslinking was not observed above $25 \mathrm{vol} \%$ ethanol.

A non-aqueous crosslinking approach was investigated where a sample was suspended in genipin-ethanol vapour in a vacuum dessicator heated to $45^{\circ} \mathrm{C}$. The fibers displayed an appreciable shift from white to navy blue, indicating genipin-gelatin bonding, and the resulting fibers were insoluble in $\mathrm{PBS}$ at $37^{\circ} \mathrm{C}$. The dissociation of the mat into discrete nanofibers for encapsulation remained an issue, as sonication produced pieces of nanofibrous mat, indicating that fiber-fiber bonding exceeded the cohesive strength of the crosslinked fiber.

As no technique for the effective crosslinking of discrete protein nanofibers could be found in the literature, the nanofiber investigation was set aside in favour of the concurrent and more promising microfiber investigation.

\subsubsection{Drawn Gelatin Microfiber Investigation and Optimization}

The formation of gelatin fibers by extrusion as originated by Yang et al. (2009), utilizes an alginate support phase for initial fiber tensile strength and cohesivity, followed by crosslinking and removal of the support phase by chelation. In order to produce fibers on a scale permitting encapsulation within a $300 \mu \mathrm{m}$ microparticle, extensive optimization was required to reduce the dehydrated fiber diameter from the literature value of $180 \mu \mathrm{m}$. The initial system, as described within the methods, utilized a collection bath and mandrel to produce and draw-out gelatin/alginate microfibers. Fibers of varying gelatin concentrations $(0-2 \mathrm{wt} \%)$ with a fixed $1.5 \mathrm{wt} \%$ alginate concentration were produced at increasing mandrel speeds until fiber breakage. The point of breakage and the diameter of the fiber obtained under those conditions are outlined in Figure 23. 

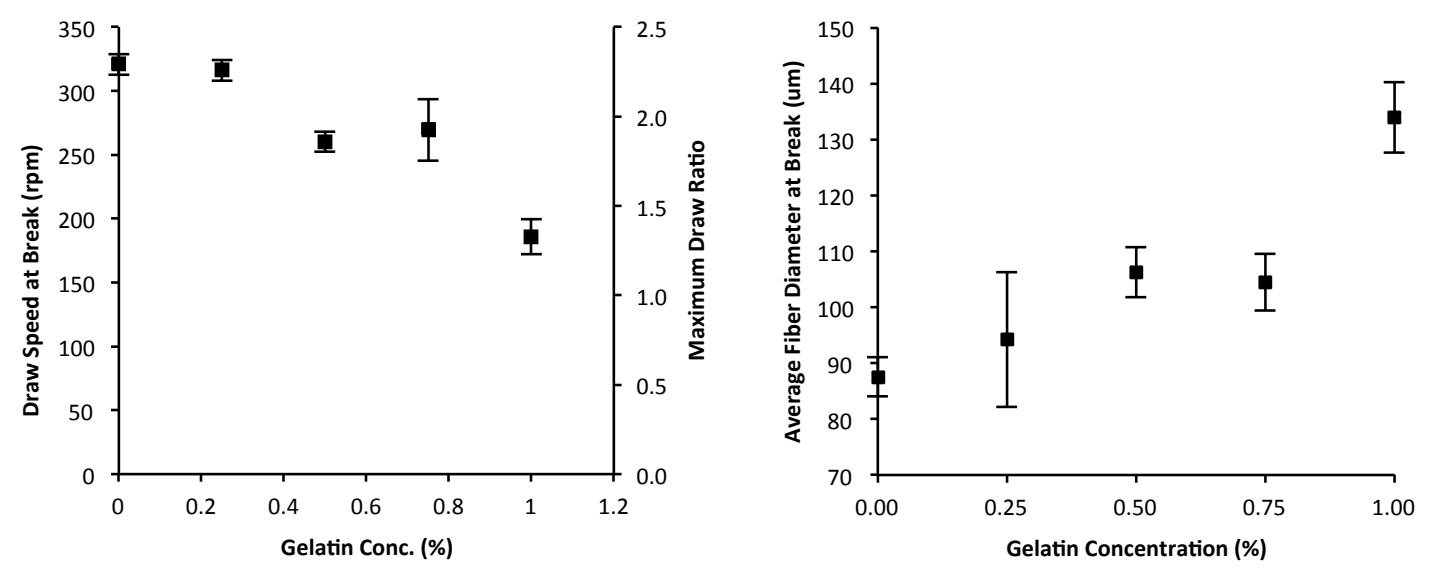

Figure 23. Relationship between gelatin concentration within mandrel-drawn $1.5 \%$ alginate microfibers and their properties. Left: Maximum mandrel rotational velocity (draw speed) and corresponding draw ratios (velocity of collection/velocity of extrusion) as a function of gelatin concentration. Right: Average fiber diameter obtained at the maximum mandrel velocity as a function of gelatin concentration. (Mean $\pm S D, n=3$ ).

Solutions containing $>1 \%$ gelatin thermoset within the syringe at room temperature and could not be used to form fibers. Increased gelatin concentration reduced the strength of the fibers produced, causing breakage at lower draw ratios with correspondingly larger diameters. Fibers produced by this method, while significantly smaller than those produced in the literature, with greater gelatin contents, were still too large to produce a microencapsulated network. In addition, without additional optimization tactics, fiber formation would necessitate very low levels of gelatin, resulting in poor mechanical properties once the alginate support phase was removed.

Therefore, the next phase of microfiber optimization had two goals - to increase gelatin content in the fiber, and further reduce fiber diameter.

Previous attempts to produce fibers with higher gelatin contents $(>1 \%)$ resulted in the solution thermosetting in the syringe before extrusion. To overcome this problem the solution-loaded syringe was heated beforehand to $45^{\circ} \mathrm{C}$, and the temperature maintained with a gel-based hot 
compress $\left(\right.$ Life Brand ${ }^{\mathrm{TM}}$ ) heated to $70^{\circ} \mathrm{C}$ in a water bath. The increase in temperature allowed for $1.5 \%$ gelatin solutions to be used in fiber spinning with $25 \mathrm{G}$ needles (Figure 24).

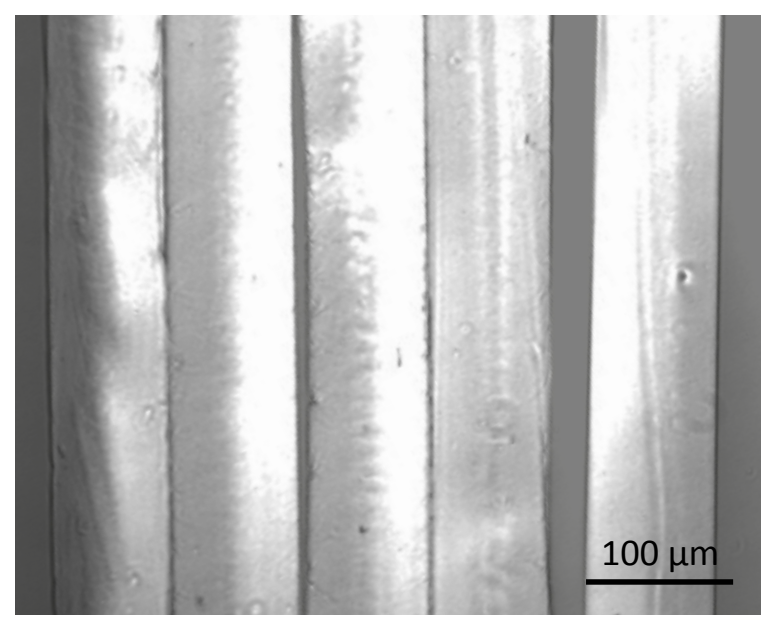

Figure 24. 1.5\% gelatin $1.5 \%$ alginate mandrel-drawn fibers, with an average hydrated diameter of $108 \pm 1 \mu \mathrm{m}(\mathrm{n}=10)$.

The further reduction of fiber size was achieved by replacing the original needles with a $0.25 \mu \mathrm{L}$ glass micropipette (100 $\mu \mathrm{m}$ ID) reduced to $1 \mathrm{~cm}$ in length and fixed within an $18 \mathrm{G}$ syringe with parafilm. This fabricated extrusion needle reduced fiber size greatly to $45 \mu \mathrm{m}$, but necessitated slowing the rotational velocity of the mandrel to prevent breakage. In addition, as the orifice had been reduced so greatly, small bubbles within the gelatin/alginate solution resulted in broken fibers, which had to be reset on the mandrel by hand, and a buildup of thermoset gelatin in the submerged micropipette caused frequent clogging.

The issue of fiber breakage and resetting was overcome by replacing the $\mathrm{CaCl}_{2}$ bath and mandrel with a vortex of $\mathrm{CaCl}_{2}$ solution. The substitution allowed for uninterrupted spinning, as the shear force imparted on the stream exiting the needle by the flow of $\mathrm{CaCl}_{2}$ solution acted as a mandrel, drawing out the fibers. As fiber-breakage was no longer as important of a concern, vortex speeds, 
and therefore draw ratios, could be increased. At this time, the problem-prone micropipette assembly was also replaced with $35 \mathrm{G}$ blunt-tipped needles ( $80 \mu \mathrm{m}$ ID).

The final alteration to the system addressed the issue of fiber inconsistency. When the stream did break, a small mass would form at the tip of the needle until the shear force on the buildup exceeded the surface tension at the needle tip. To overcome this difference in surface tensions while improving the laminar nature of the vortex at higher rotational speeds, $1.5 \%$ gelatin was added to the collection bath. The bath was also heated to $45^{\circ} \mathrm{C}$ to prevent any thermosetting within the injection needle, further improving consistency. The use of gelatin to increase the viscosity of the collection bath avoided the inclusion of an additional contaminating component, such as dextran, into the solution.

With the modified vortex-extrusion design, coupled with genipin crosslinking, chelation, sonication, and settling outlined in the methods, gelatin fibers were produced with an average hydrated diameter of $22.3 \pm 0.4 \mu \mathrm{m}$ and average length of $223 \pm 13$ um (Figure 25).
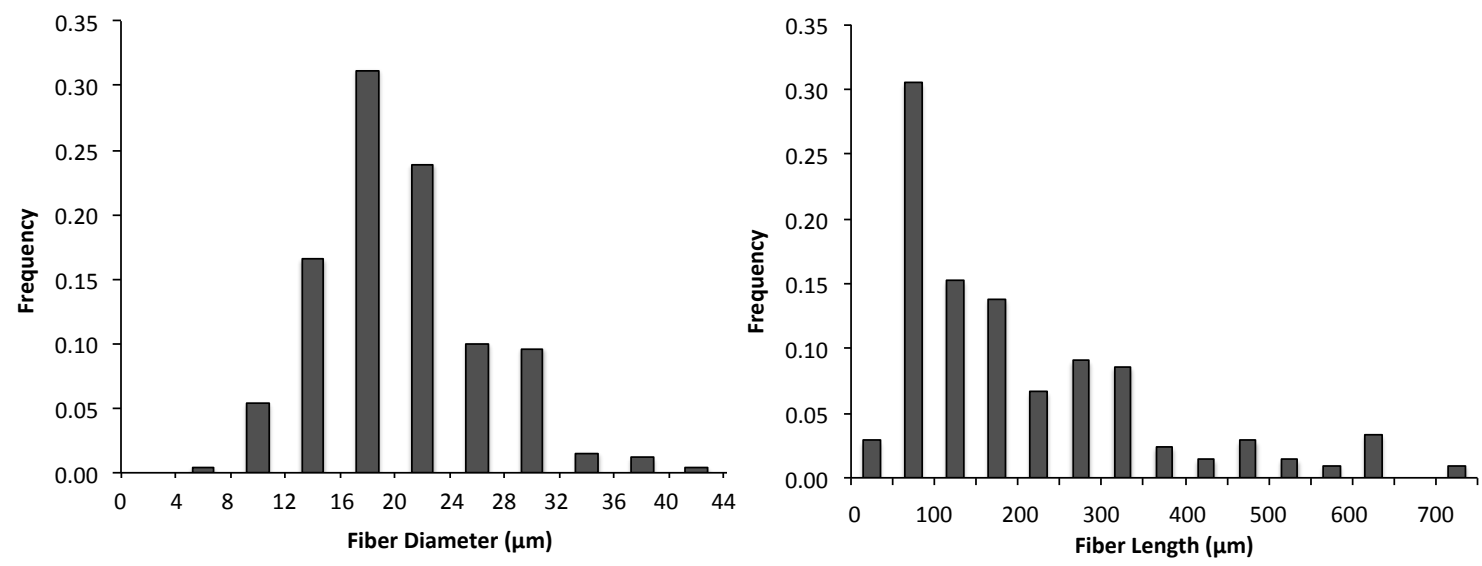

Figure 25. Dimensions of rehydrated genipin-crosslinked $1.5 \%$ gelatin microfibers. Left: Hydrated diameter, av = 22.3 $\pm 0.4 \mu \mathrm{m}(\mathrm{n}=296)$; Right: Length, av $=223 \pm 13 \mu \mathrm{m}(\mathrm{n}=214)$.

The optimized fibers were imaged by scanning electron microscopy (SEM) in a dehydrated state, showing evidence of potential alignment and fibril formation within the microfiber (Figure 26). 

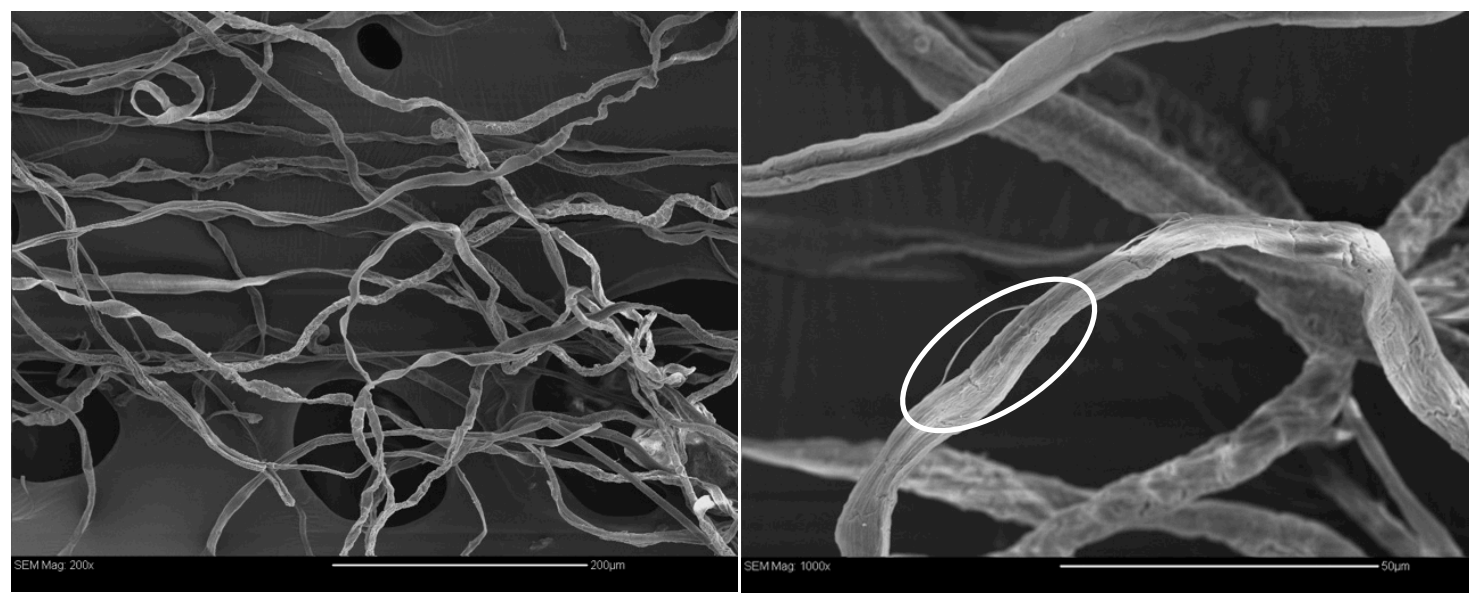

Figure 26. SEM images of dehydrated microfibers. Left: Crosslinked fibers are discrete and uniform (scale bar represents $200 \mu \mathrm{m}$ ). Right: Evidence of alignment and fibril formation within the microfibers (scale bar represents $50 \mu \mathrm{m}$ ).

\subsubsection{Fiber Integrity and Crosslinking}

The bonding of gelatin with genipin produces a distinct navy blue colouration $(610 \mathrm{~nm})$. The evolution of the visible spectrum for a $10 \%$ gelatin sample over a period of 120 minutes is illustrated in Figure 27 alongside a plot indicating the linear nature of absorbance at $610 \mathrm{~nm}$ as a function of time for three gelatin concentrations. 

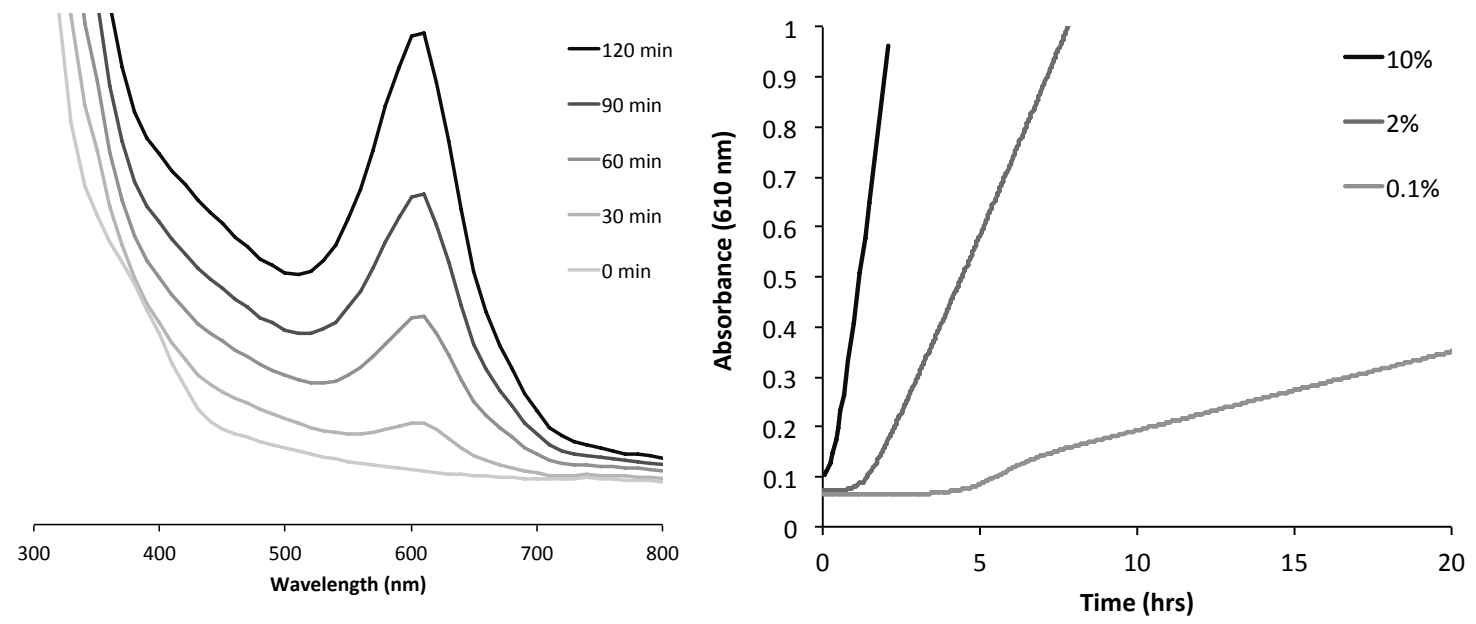

Figure 27. Genipin-gelatin crosslinking and absorbance at $610 \mathrm{~nm}$. Left: Change in the absorbance within the visible spectrum for $10 \%$ gelatin crosslinking with genipin over 120 minutes. Right: Absorbance at $610 \mathrm{~nm}$ as a function of time for three different gelatin concentrations.

As soluble xenogenic proteins have the potential to elicit an immune response, hindering the immunoisolation of the islets, tests were performed on the crosslinking efficiency and fiber integrity under culture conditions.

Microfibers crosslinked in $0.25 \mathrm{wt} \%$ genipin solutions in $\mathrm{pH} 8.0$ Tris buffer for 48 hours were analyzed by ninhydrin assay for the presence of primary amines, the reactive site for genipin crosslinking. The crosslinked samples exhibited a $65 \pm 8 \%$ crosslinking efficiency $(n=4)$, defined as the reduction in primary amines relative to uncrosslinked controls (Equation 1). This is comparable to the maximum observed crosslinking efficiency of $75 \pm 2 \%$ for the genipin-gelatin system (Yao, 2004).

Encapsulation-ready microfiber samples were incubated at $37^{\circ} \mathrm{C}$ for 70 days in sterile PBS to measure uncrosslinked or hydrolyzed gelatin leaching from the fibers. The supernatant was analyzed by BCA assay, which indicated a negligible loss of $<0.01 \%$ of the mass of the fiber sample over 70 days (Figure 28). 


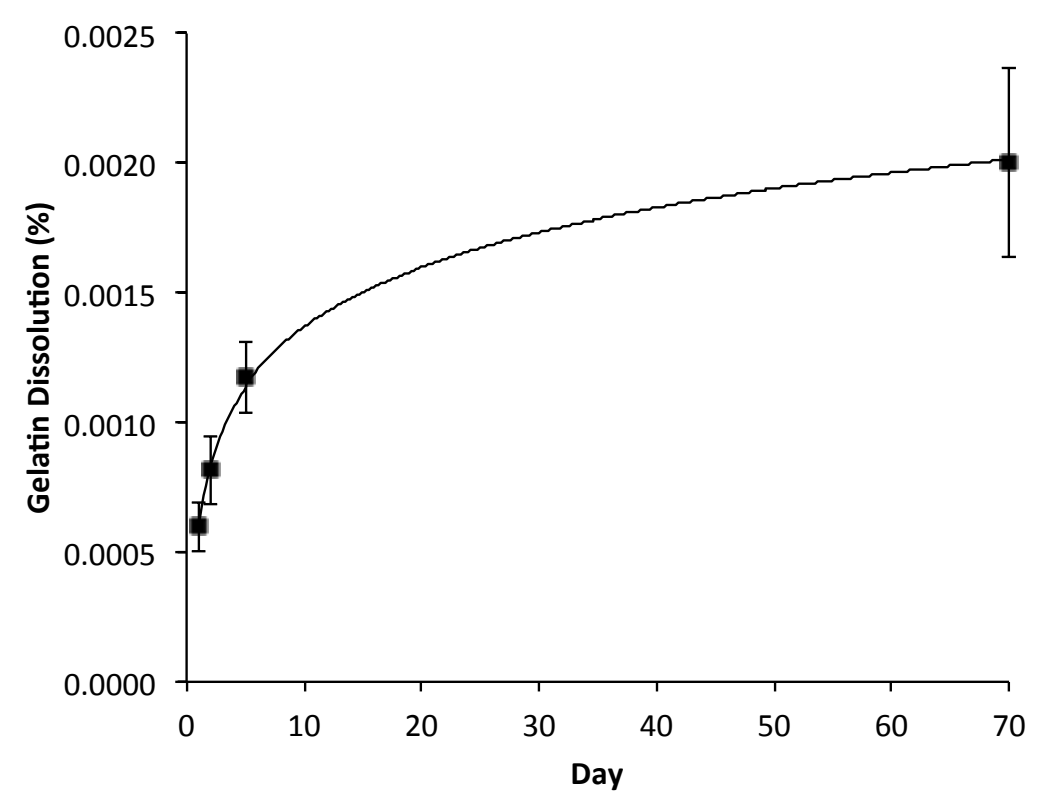

Figure 28. Gelatin detected by BCA over a 70 day incubation of fibers in $37^{\circ} \mathrm{C}$ sterile $\mathrm{PBS}$. $($ Mean \pm SD, $n=3$ ).

\subsection{Particle Production}

\subsubsection{Emulsion Encapsulation Optimization}

The initial approach to the production of a microfibrous matrix within a microparticle was the internal gelation emulsion system. The emulsion system appeared ideal as it would concentrate fibers within the center of the particles and weave the fibers into a tight matrix. Varying the emulsion times and fiber concentrations allowed for a wide variety of particles to be produced from the initial stock of large diameter microfibers (Figure 29). 

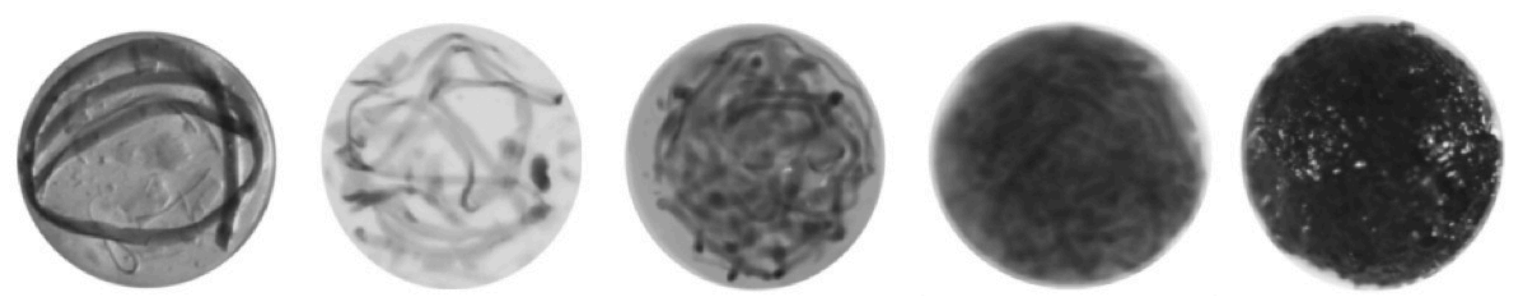

Figure 29. A distribution of fiber densities from different external gelation emulsion systems. Particles ranged from $600 \mu \mathrm{m}$ to over $3 \mathrm{~mm}$ in diameter.

A preliminary trial of islet encapsulation using islet-sized $1.08 \mathrm{~g} / \mathrm{mL}$ FITC-labeled gelatin particles indicated that simulated islets were readily encapsulated within the fibrous emulsion (Figure 30).

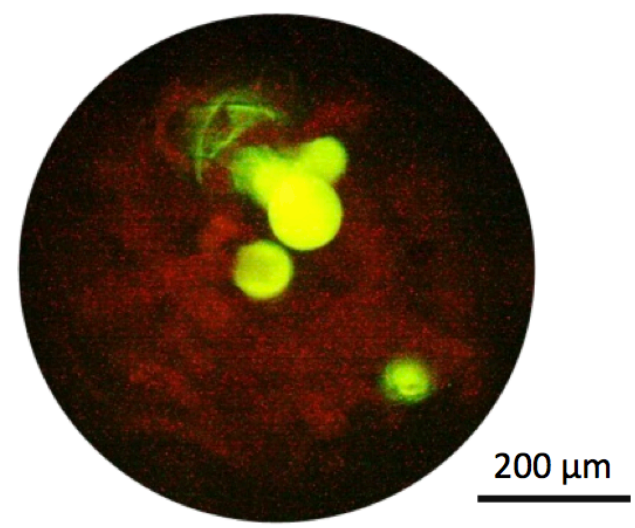

Figure 30. FITC-labeled islet-like particles (green) encapsulated within a microfiber network (red).

In a conventional emulsion, an equilibrium between particle coalescence and disruption is observed, producing a broad normal distribution of particle diameters. However, in a fiber-laden emulsion, a bimodal system develops. Upon coalescence of droplets, the fiber networks interpenetrate and are centralized within the droplet (Figure 31). Shear force on the surface of the droplet draws off the fiber-free alginate, further concentrating the fibers. Fiber core coalescence continues until the system is ionically crosslinked with acidification, resulting in a bimodal distribution of large dense particles and a large number of fine fiber-free particles (Figure 32). 


\section{Traditional Emulsion Equilibrium}
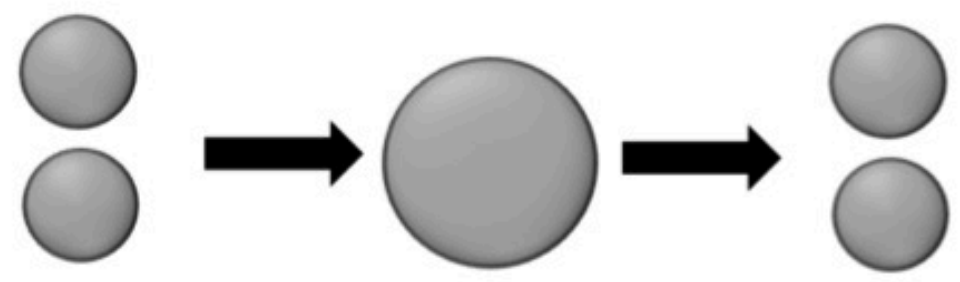

\section{Fiber-laden Emulsion}
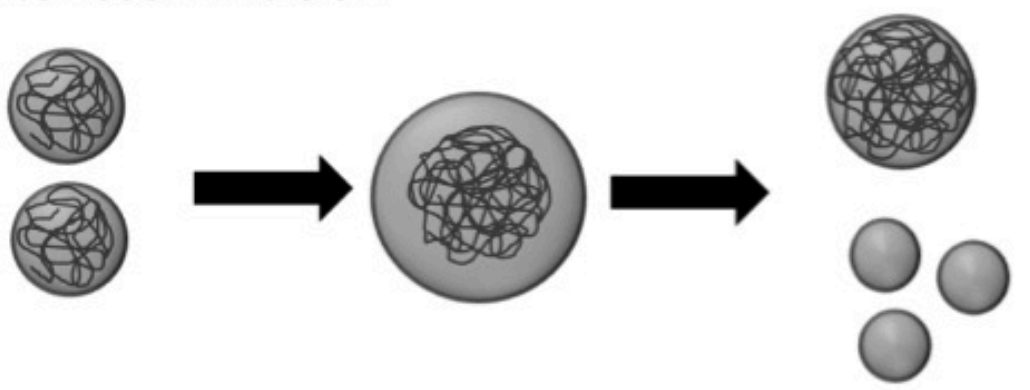

Figure 31. Comparison between traditional and fiber-laden emulsions

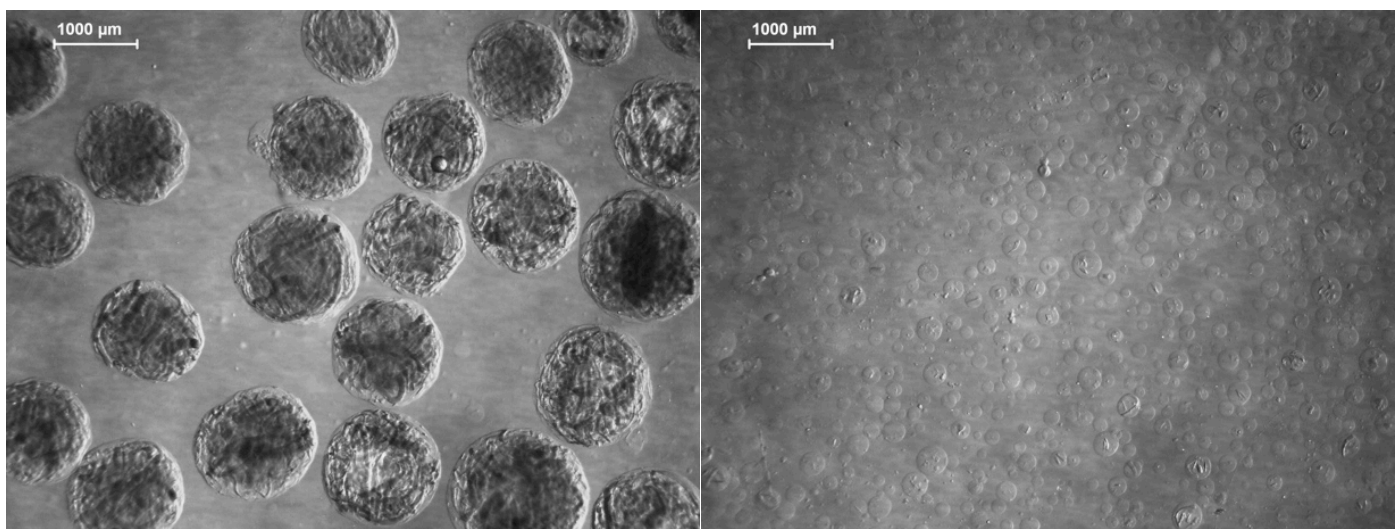

Figure 32. Bimodal distribution of large, dense particles (left) and small fiber-free particles (right) produced by an 8 minute emulsion (scale bars represent $1000 \mu \mathrm{m}$ ).

Issues with the multivariate optimization of the emulsion system coupled with the intrinsically broad particle size distribution from emulsion systems led to the investigation of electrostaticmediated dropwise particle production. 


\subsubsection{Electrostatic-Mediated Dropwise Encapsulation}

Electrostatic-mediated dropwise encapsulation produced fiber-laden particles with a narrower size distribution and allowed for the production of much smaller particles within the $<300 \mu \mathrm{m}$ range of interest. Additionally, as the inclusion of fibers was a modification to a widely implemented cytocompatible encapsulation process, optimization for primary islet viability was not required.

Following an investigation of particle size as a function of fiber concentration and extrusion speed, islet-laden particles containing $40 \mathrm{vol} \%$ fiber concentrate in $1.6 \mathrm{wt} \%$ purified alginate were produced at $2 \mathrm{~mL} / \mathrm{h}$ with a mean diameter of $294 \pm 4 \mu \mathrm{m}(\mathrm{n}=260)($ Figure 33).

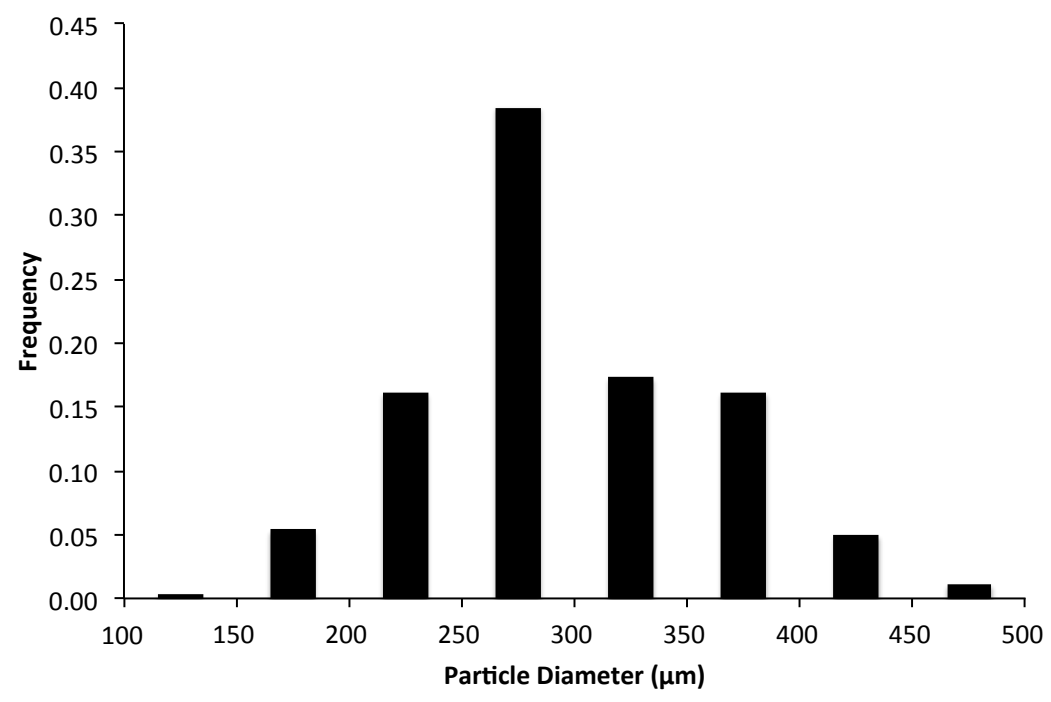

Figure 33. Electrostatic-mediated dropwise encapsulation particle size distribution.

Figure 34 illustrates the two different encapsulation systems used in the endeavor to produce $<300 \mu \mathrm{m}$ alginate microparticles containing an interwoven protein network. As can be observed, the initial emulsion system produced particles with an interwoven network of fibers, but with 
insufficient control over the size distribution. By comparison, the particles from the electrostaticmediated dropwise technique are more uniform in size and composition, with an average particle size under the $300 \mu \mathrm{m}$ target.
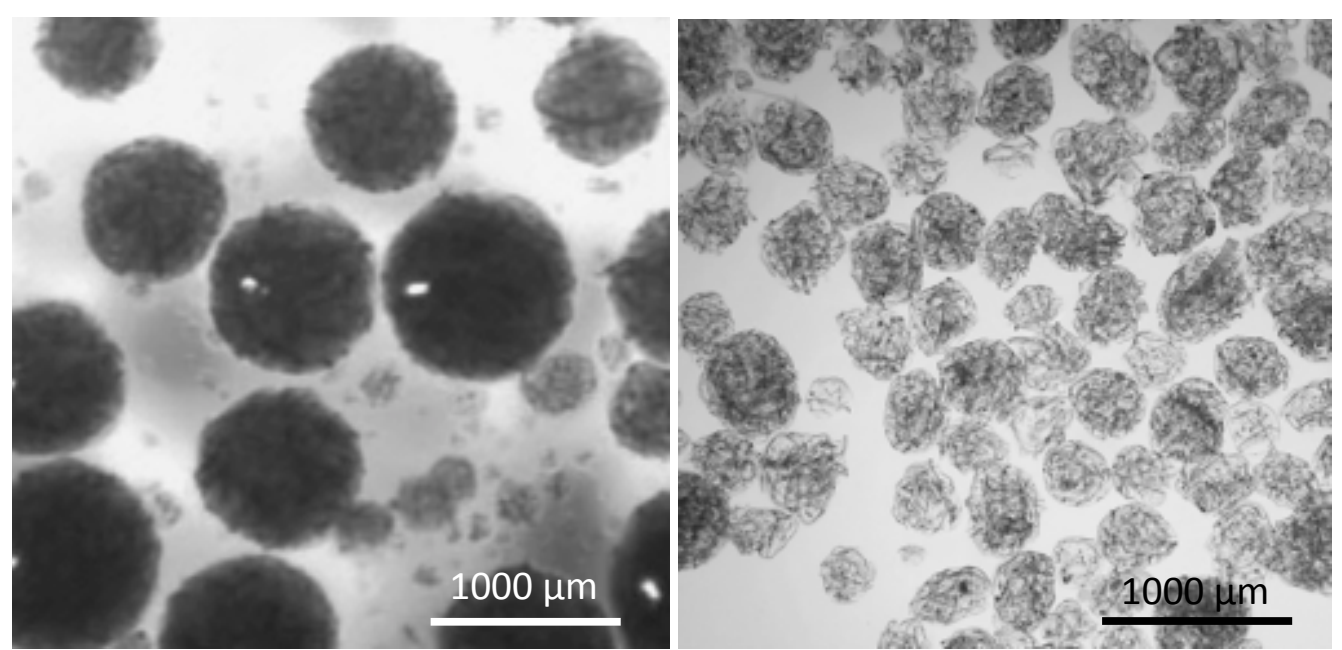

Figure 34. Alginate particles containing gelatin networks produced by both techniques, on a common scale. Left: emulsion, Right: electrostatic-mediated dropwise.

\subsection{Preliminary In Vitro Investigation}

\subsubsection{Islet Isolation}

Islets were isolated from nine male Sprague Dawley rats with a collagenase V digestion, yielding an average islet size of $134 \pm 3 \mu \mathrm{m}(\mathrm{n}=266)$ (Figure 35$)$. 

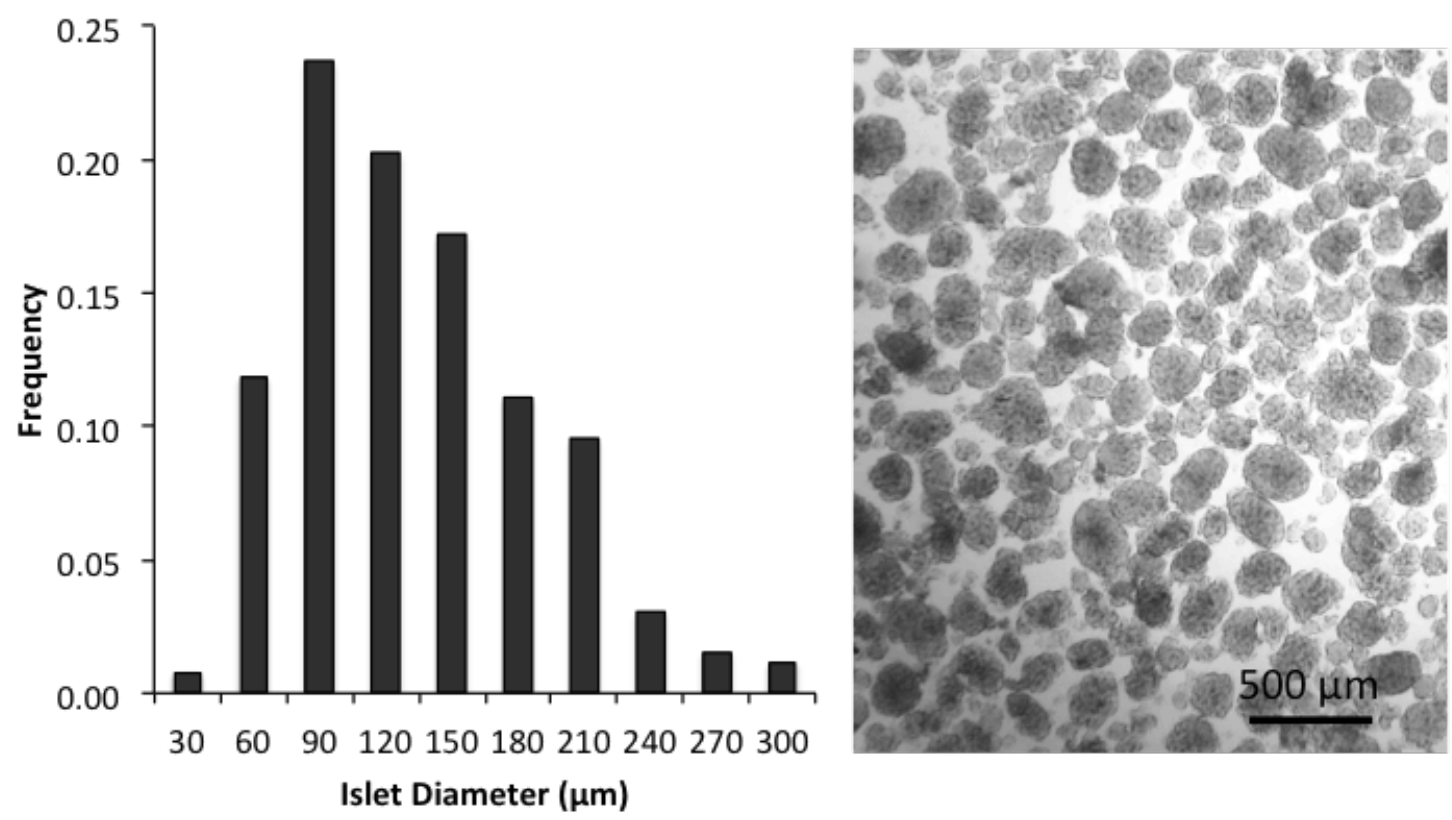

Figure 35. Left: Size distribution of isolated islets. Right: Image of isolated islets.

More than 400 islets were recovered on average from each rat, although many of the recovered islets were significantly smaller than the $150 \mu \mathrm{m}$ sphere used to standardize the "islet equivalent". The extraction protocol coupled with a EuroFicoll density gradient produced a highly purified islet sample, simplifying the inclusion and exclusion handpicking which was performed on each sample. In the event that bile-duct infusion was unsuccessful, the pancreases were injected with collagenase solution. When compared to successfully infused pancreases, the digestion of the injected pancreases was less effective and the resulting islet recovery and sample purity were lower. Following the purification in the EuroFicoll density gradient, the residual islet presence in the pellet was determined by DTZ staining. The concentration of residual islets was consistently insignificant, with only the occasional islet visible in the cell mass, often associated with small, undigested masses of tissue. 


\subsubsection{Viability and Cohesion}

Viability was assessed with propidium iodide (PI) - acridine orange (AO) staining of 100 particles for both the fiber-laden particles and the fiber-free control and viewed under a fluorescent microscope with a blue excitation filter $(490 \mathrm{~nm})$. Under fluorescent microscopy, viable cells stain green and dead cells stain red. The viability gradient was divided into 5 classifications outlined in Figure 36 (Langlois, 2009).

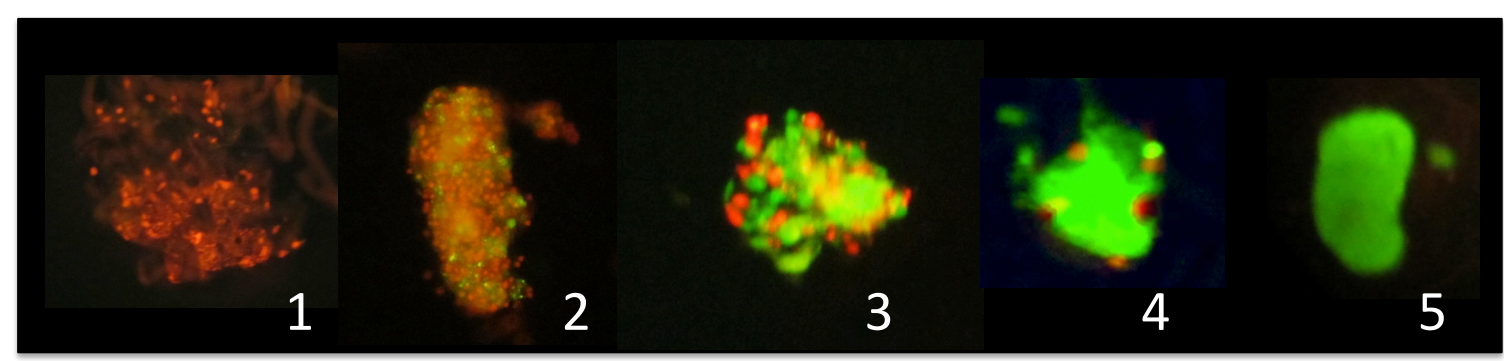

Figure 36. AO/PI viability assay classification. 1 - completely red/colourless, 2 - more than $50 \%$ red/colourless, 3 - more than $50 \%$ green, 4 - less than $10 \%$ red/colourless cells, 5 completely green.

Islets ranked 1 or 2 were considered non-viable, while those in the 3-5 range were considered viable. While this non-viable/viable classification system is based on the ability of islets to return from a state of partial necrosis, it artificially inflates the true state of the system as the average rankings approach 50\% viability (Robitaille, 2003). For this reason both the non-viable/viable and average viability rankings for the fiber-laden particles and fiber-free controls over 21 days are displayed in Figure 37. Displaying the average viability ranking also allows for the calculation of statistical significance, which is exhibited at 7,14 , and 21 days $(\mathrm{p}<0.01)$. 

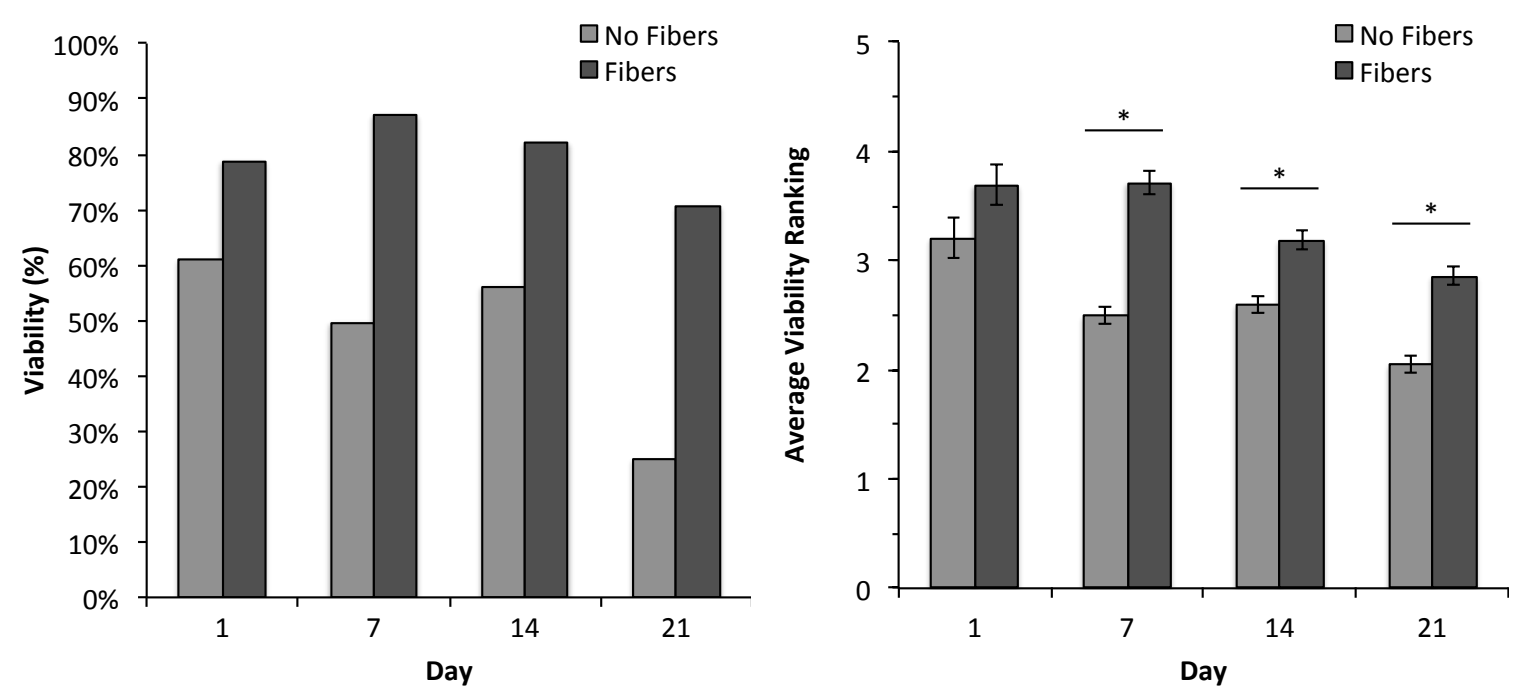

Figure 37. Viability of rat islets encapsulated within fiber-laden and no-fiber control particles over a period of 21 days. Left: Viability assessment based on the 1-2/3-5 nonviable/viable classifications. Right: Average viability ranking, (mean $\pm \mathrm{SE}$, day 1: $n>50$, days 7-21: $n>100)$, *statistically different $(p<0.01)$.

One issue encountered during the viability staining was the loss of the propidium iodide (red) signal from the non-viable cells following day 1 . While some cells did continue to stain red, there was a significant cell mass that could only be visualized by light microscopy. To overcome this, and avoid artificially raising the viability ranking, the slides were illuminated with white backlight reduced sufficiently to allow visualization of the entire cell mass as well as the fluorescent signal. The percentage of acridine-orange (green) signal was determined with respect to the total cell mass, as opposed to the fluorescent cell mass.

The cause of the loss of propidium iodine signaling is believed to be due to double stranded nucleic acid degradation and/or diffusion following cell death. At day 1, the cell death observed was recent, and therefore the double stranded nucleic acid content within the cells was still concentrated and capable of intercalating with PI. However, by day 7, the nucleic acids in the 
days-old necrotic cells had degraded and/or diffused into solution such that it could no longer be visualized by PI, and thus appeared colourless under fluorescent excitation.

The PI/AO staining protocol provided additional information on the cohesivity of the cells within viable islets over the 21-day investigation. Upon isolation, islets are composed of a single dense aggregate of viable cells, appearing as a single cohesive mass under viability staining protocols. However, at days 14 and 21 a trend towards a disrupted distribution was observed in both the fiber-laden and control particles, however this trend was more pronounced in the control particles which exhibited a $31 \%$ incidence of disruption at day 21 compared to only $8 \%$ for the fiber-laden particles (Figure 38). As cell-cell contact is so important in the regulation of glucose secretion, the disrupted distribution of cells within the islet would likely be very detrimental to islet function.
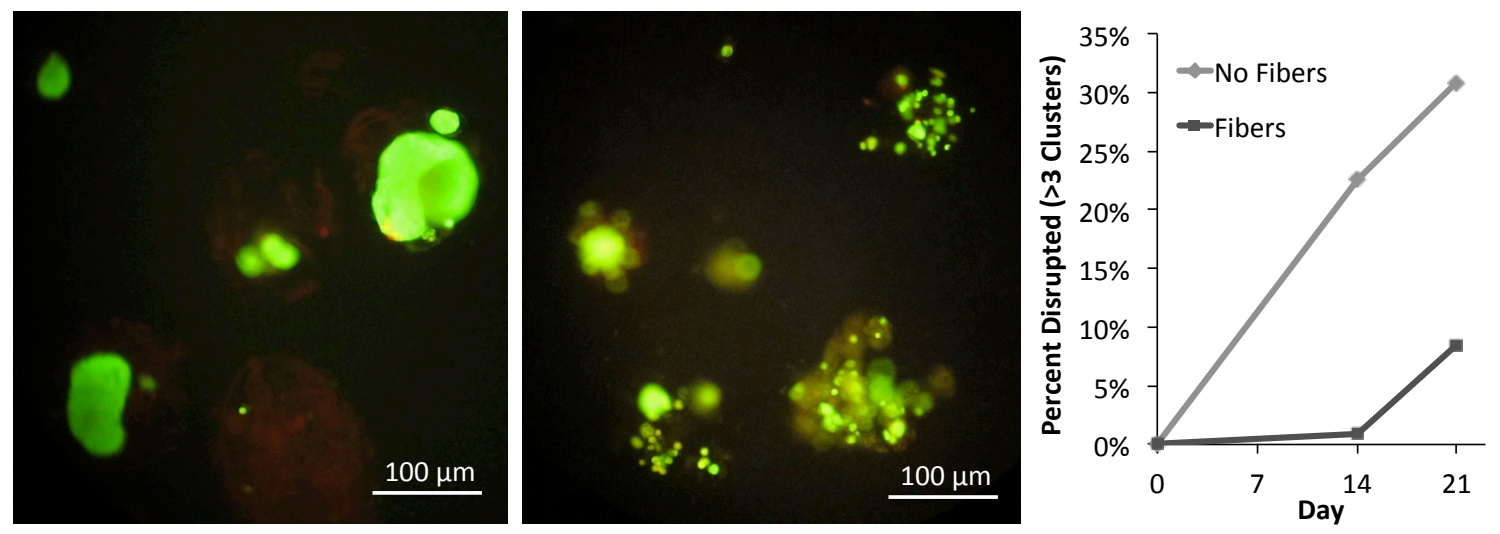

Figure 38. Left: Cohesive islet distribution under AO/PI staining, Center: Disrupted islet distribution with ( $>3$ clusters), Right: Percent of viable islets disrupted as a function of time $(\mathbf{n}>100)$.

Direct interaction between the islets and the fibers was observed in the short time before encapsulation and within particles after 24 hours. Instances of cellular adhesion onto gelatin fibers were observed after less than an hour of co-incubation in the residual alginate solution 
containing both fibers and islets. Figure 39 illustrates two additional islet-fiber interactions which, while not representing the predominant trend, confirm the potential for adhesion. In the image to the left stained by H\&E, a single cell can be observed adhered directly to a fiber. The fluorescent image to the right was generated when a fiber-laden particle with AO/PI viability staining was mounted under a coverslip. As the particle was flattened by the coverslip, the encapsulated islet stretched as well because it was adhered to the fiber matrix. In the image we see that the zone of adhesion to the fiber was extensive, and contains the only cells staining distinctly viable.
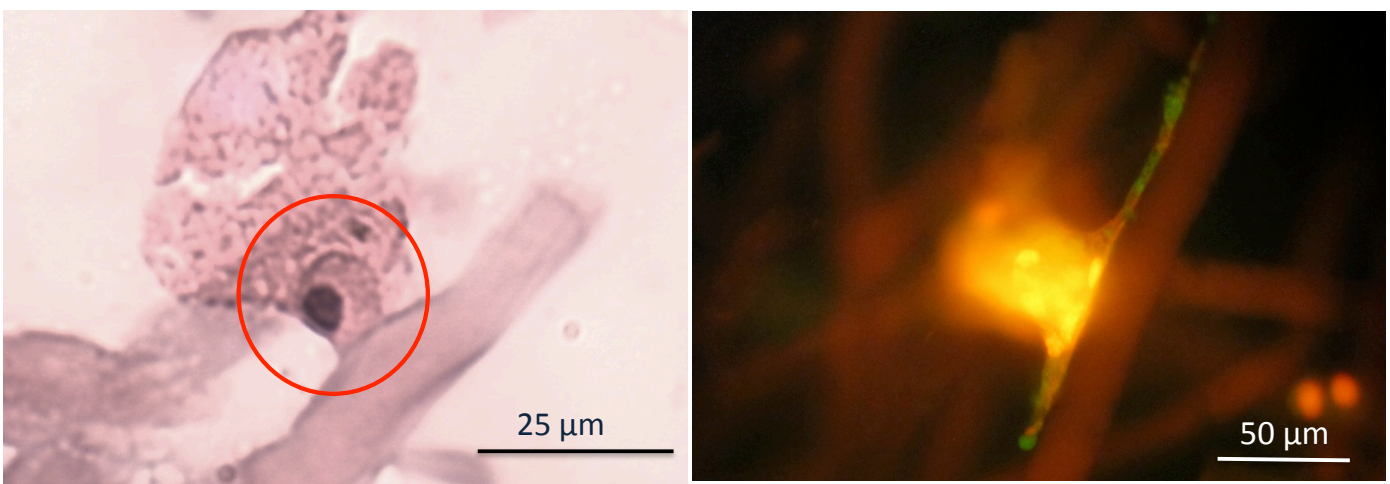

Figure 39. Islet adhesion to encapsulated gelatin fibers. Left: A single $\beta$-cell adhered to a gelatin fiber, observed in a section stained with $\mathrm{H} \& \mathrm{E}$. Right: An islet adhered to a gelatin fiber observed under $\mathrm{AO} / \mathrm{PI}$ viability staining.

\subsubsection{Metabolic Activity}

Metabolic activity was measured with a CellTiter $96^{\circledR}$ Aqueous One Solution Cell Proliferation Assay (Promega Corp) on 50 particles in triplicate on day 1, and 100 particles in triplicate thereafter. Metabolic data was collected at 4 hours for days 1, 7, 14 and 21, and additionally at 24 hours for days 7, 14, and 21 (Figure 40). 

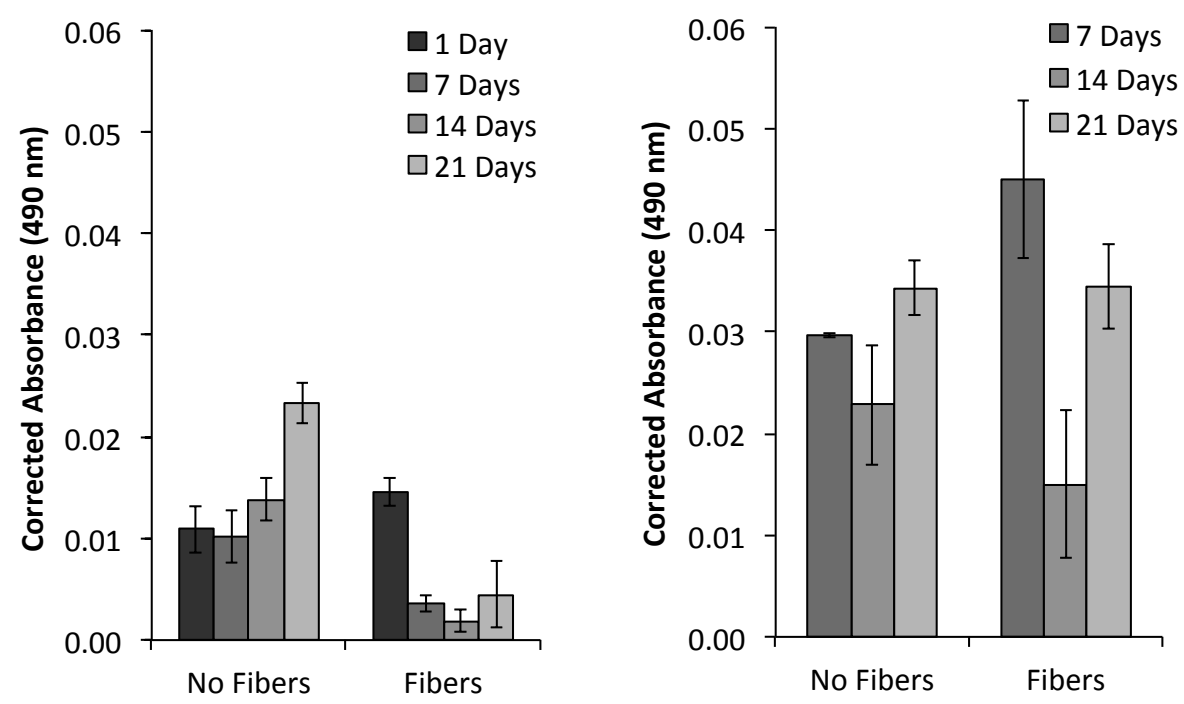

Figure 40. Corrected absorbance for the CellTiter $96^{\circledR}$ Aqueous One Solution Cell Proliferation assay after different culture durations over 21 days. Left: 4 hours, Right: 24 hours. (Mean $\pm \mathrm{SE}, \mathbf{n}=3$ )

The absorbance readings generated by the metabolic assay were very low, averaging only $13 \pm 4$ $\%$ above the blank wells with islet-free and fiber-free control particles at 4 hours, and $21 \pm 2 \%$ at 24 hours. Therefore, despite increasing to 100 particles per well, and extending the incubation period, the viable cellular density was insufficient to generate statistically significant data. The different trend observed between the 4 hour and 24 hour data may be due to the initial absorption of the substrate or product onto the protein fiber surface, as the substrate was available in excess. The accumulation of product in the periphery of the islets was observed, as the islets transitioned to a distinctly pink colouration by the end of the assay. However, parallel investigations with barium alginate microparticles containing immortalized discrete cells indicated that there was no issue with diffusion in the barium alginate encapsulation system. The trend within the majority of the metabolic data does not correlate to the viability or insulin secretion data, which both decrease with time. 


\subsubsection{Glucose-Induced Insulin Secretion}

Glucose-stimulated insulin secretion measures the ability of islets to adjust their insulin secretion to changing levels of glucose in their environment, a physiologically important response to hypo/hyperglycemia. Insulin secretion was tested by a two-stage glucose tolerance test. At each time point all 24 samples of 50 islets were first incubated for an hour in RPMI media containing a basal level of $3.3 \mathrm{mM}$ glucose and insulin secretion was measured by ELISA (Figure 41). Following the basal test, samples were washed and tested in triplicate at $3.3,5.5,11$, or $16.5 \mathrm{mM}$ glucose RPMI for an additional hour (Figure 42). The ratio of the insulin secretions at a test concentration over a basal concentration is referred to as the insulin stimulation index (Figure 43). At each time point, each triplicate of wells was tested with the same glucose concentration.

Observing Figure 41, basal insulin secretion increases from day 1 to day 7, indicating that islets are under stress conditions on day 1 due to the extraction and encapsulation, and recover by day 7. Beyond day 7, basal insulin secretion drops significantly, indicative of islet death and decreased metabolic activity. Under test conditions, increased insulin secretion was not consistently observed (Figure 42), resulting in insulin secretion indices under 1 (Figure 43). Decreased insulin secretion under test conditions may not be a proper reflection of the insulin response. If significant retention of insulin occurred in the hydrogel beads from the conditioned culture medium, despite the wash, any diffusion into the small volume of basal media would have a significant effect over the short one-hour culture period, particularly at the basal glucose concentration when insulin secretion is at its lowest. This diffusion would artificially inflate the basal secretion, decreasing the stimulation index.

In the absence of contamination, insulin secretion increases linearly from approximately $20 \mathrm{ng} / 50$ 
islets/h for $4 \mathrm{mM}$ basal glucose to approximately $85 \mathrm{ng} / 50$ islets/h for $16.5 \mathrm{mM}$ glucose (Nolan, 2009). Therefore, the basal secretion observed on days 1 and 7 in Figure 41 is higher than expected while the secretion under test conditions in Figure 42 is lower than expected for the higher glucose concentrations.

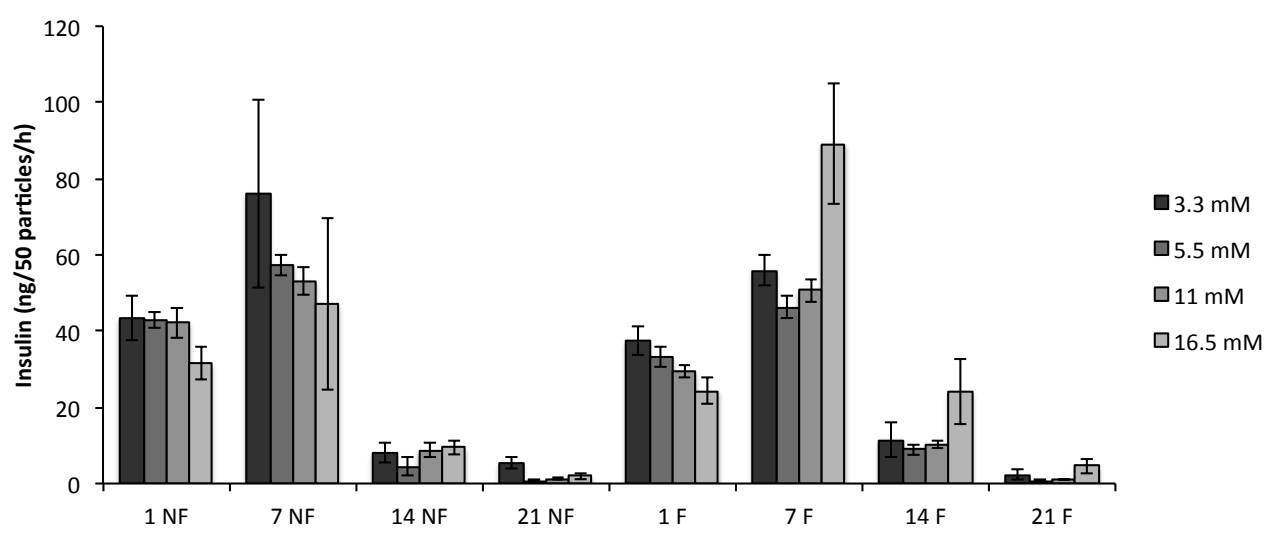

Figure 41. Insulin secretion over a 1-hour incubation in $3.3 \mathrm{mM}$ glucose RPMI at days 1, 7, 14, and 21 for no-fiber (NF) and fiber-laden (F) particles divided into their 3.3, 5.5, 11, and $16.5 \mathrm{mM}$ test groups for comparison with data in Figure 42. (Mean $\pm \mathrm{SE}, \mathrm{n}=3$ )

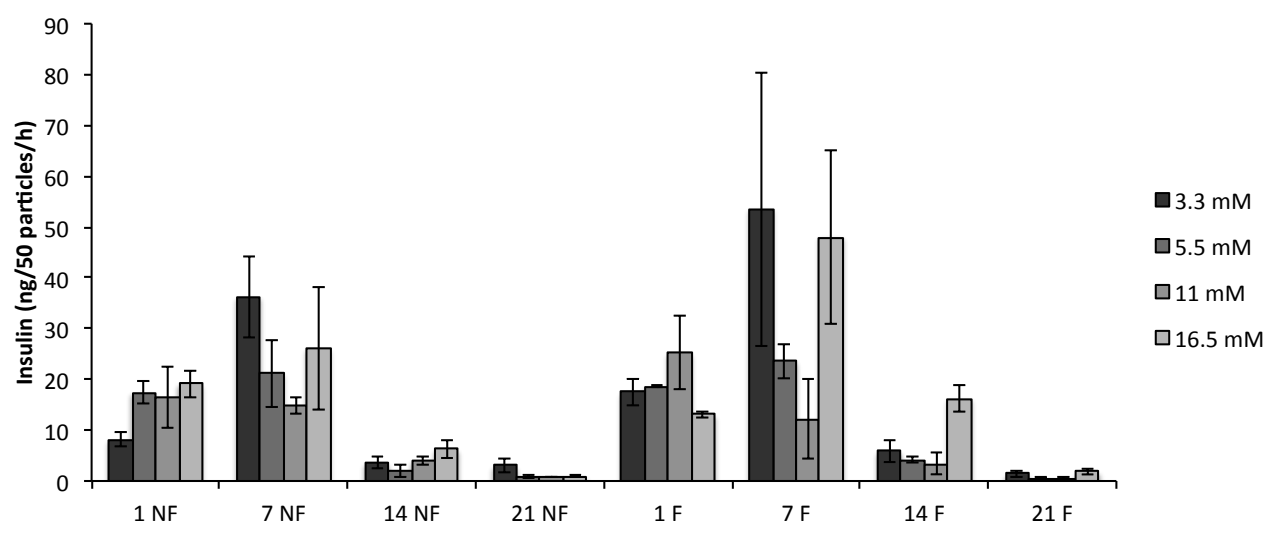

Figure 42. Insulin secretion over a 1-hour incubation in 3.3, 5.5, 11, or $16.5 \mathrm{mM}$ glucose RPMI immediately following a $3.3 \mathrm{mM}$ glucose incubation (Figure 41) at days 1, 7, 14, and 21 for no-fiber (NF) and fiber-laden (F) particles. (Mean $\pm \mathrm{SE}, \mathrm{n}=3$ ) 


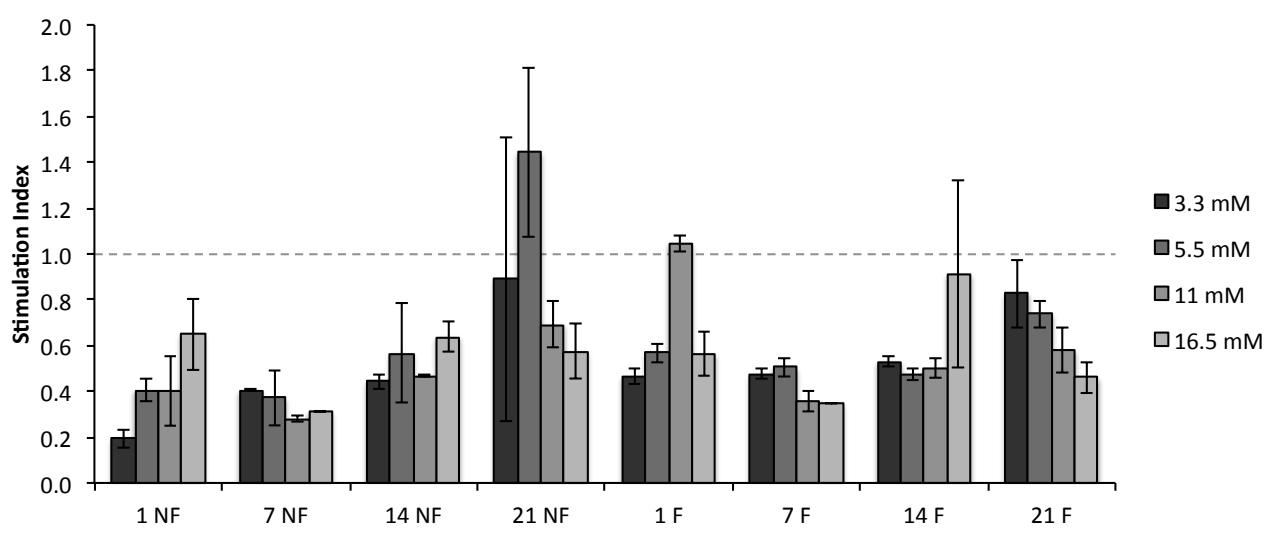

Figure 43. Stimulation index for 50 particles cultured under different test concentrations of glucose (Figure 41, Figure 42) at days 1, 7, 14, and 21 for no-fiber (NF) and fiber-laden (F) particles. (Mean $\pm \mathrm{SE}, \mathbf{n}=\mathbf{3}$ )

\subsubsection{Apoptosis}

Apoptosis was determined from nuclear morphology visualized by Hoechst 33258 fluorescent nuclear staining. Figure 44 displays the distribution of nuclear morphologies observed within the sections. Nuclei were classified as either normal/round or condensed/irregular. The percentage of condensed/irregular nuclei observed at each time point is shown in Figure 45.

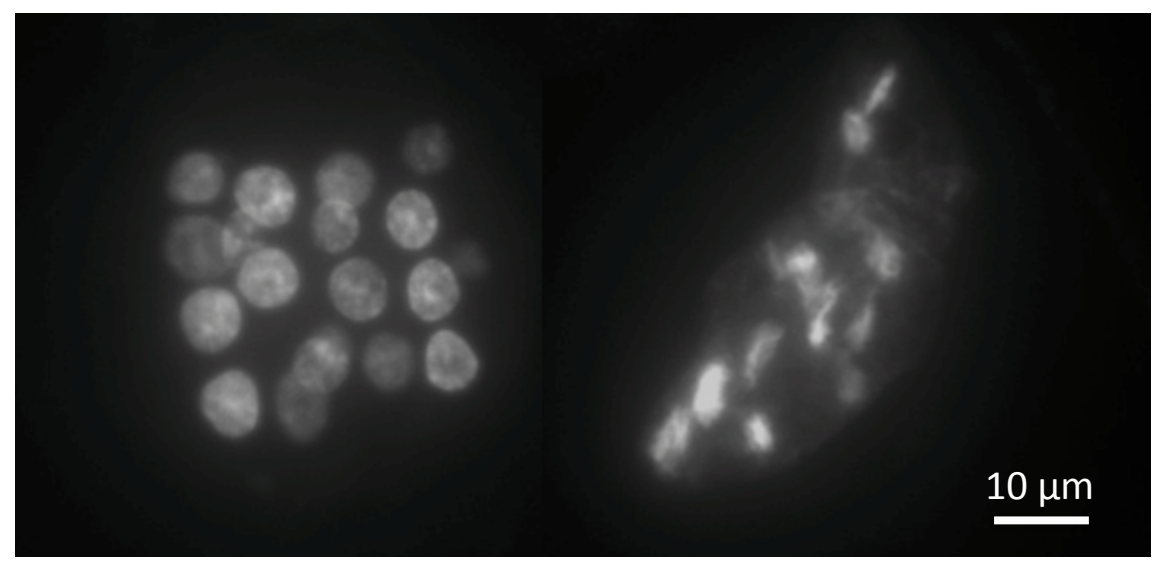

Figure 44. Rat islet sections stained with Hoechst 33258 and observed with a cyan excitation filter. Left: normal/round nuclei, Right: condensed/irregular nuclei. 


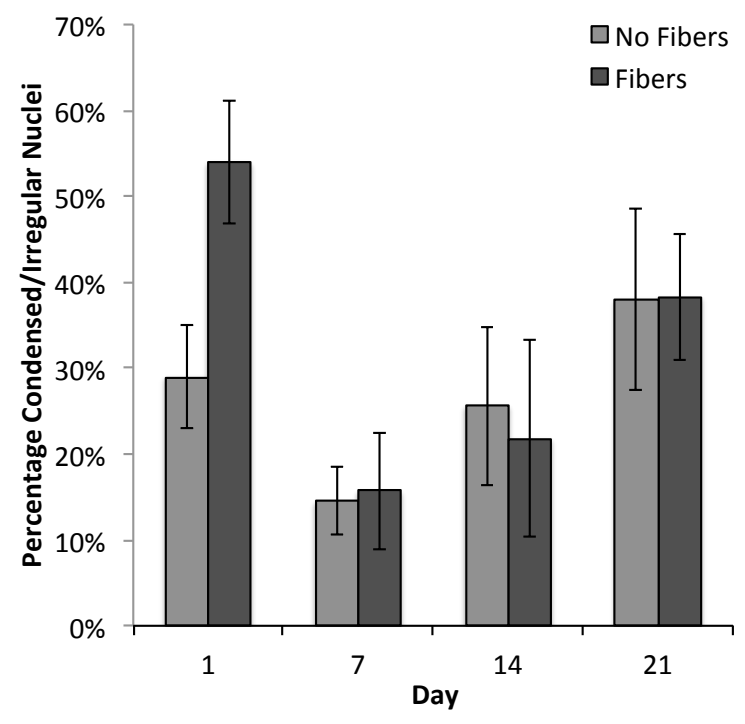

Figure 45. Nuclei in Hoechst-stained sections with a condensed/irregular morphology over 21 days for fiber-laden particles and the fiber-free controls. (Mean $\pm S E, n=7$ to 17)

The high incidence of condensed/irregular nuclei at day 1 can be attributed to the stress associated with extraction and encapsulation, additionally the difference between the two data sets is not significant $(\mathrm{p}>0.05)$. The data for days $7-21$ indicate no difference between the fiberladen and control groups, but an increasing trend, agreeing with the decreasing trend observed in viability. The large degree of uncertainty within the data is due to a low sample size (7-17 islets containing an average of $23 \pm 20$ nuclei), as a result of issues with sample preparation and sectioning.

Low melting point agarose was used to contain the microparticles during sample preparation. However, the differing rigidities of paraffin, low melting point agarose, and alginate resulted in particles separating from the section and being lost, resulting in characteristic voids in the agarose and a low particle count in the sample (Figure 46). 


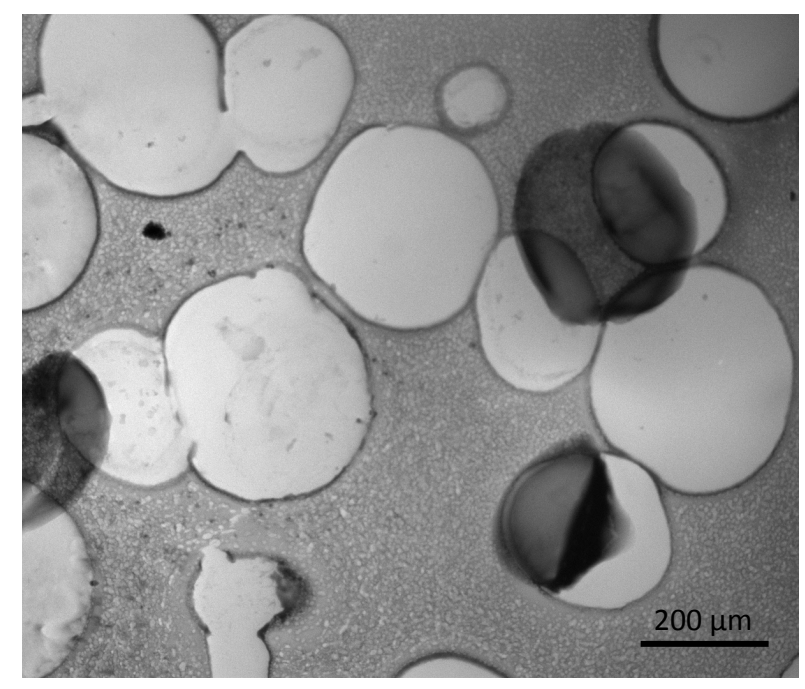

Figure 46. Particle loss during sectioning and slide preparation. Displaced particle sections (dark grey) visible on a background of low melting point agarose (light grey) and voids (white) initially formed by particles. 


\section{Chapter 6}

\section{Conclusions and Future Work}

The production of discrete crosslinked protein microfibers was optimized, reducing the hydrated fiber diameter to $22.3 \pm 0.4 \mu \mathrm{m}$, while making the process more reliable and less labour intensive. The optimized microfibers were encapsulated within $294 \pm 4 \mu \mathrm{m}$ barium alginate microparticles by an electrostatic-mediated dropwise extrusion system. The encapsulated microfibers formed a novel system incorporating an immunoisolated, interwoven, fibrous gelatin network with the ability to envelop a cellular aggregate far larger than the network pore size. Islets were extracted from Sprague Dawley rats and encapsulated within the particles for a 21-day preliminary in vitro study. Fluorescent viability staining and light microscopy indicated a significant increase in viability, a trend towards retained islet cohesion, and islet-fiber adhesion. Investigations into insulin secretion, metabolic activity, and DNA quantification require further investigation at higher viable cell counts.

The primary contribution of this body of work to the field of tissue engineering is the development of a method to produce discrete gelatin fibers in the $20-100 \mu \mathrm{m}$ size range. There are currently no published techniques for spinning fibers in this intermediate range, finer than mandrel-drawn fibers $(>100 \mu \mathrm{m})$ and larger than electrospun fibers $(<1 \mu \mathrm{m})$. The work on emulsion encapsulation has the potential to be optimized into a very promising technique. However, the issues with emulsion kinetics must be overcome to produce reproducible, relatively monodisperse particles in the $>300 \mu \mathrm{m}$ range. This work will hopefully act as a foundation for the development of the one-step aggregate encapsulation method. 


\section{Key Points To Consider For Future Work}

In the future, glucose-tolerance testing should be performed with a perfusion system, as opposed to static culture. Static culture was selected due to the large number of samples and concentrations to be analyzed, along with the perceived simplicity of the assay. However, issues with incomplete particle washing may have led to the inconsistencies between the observations and the expected values from literature. By monitoring a time-course of the perfused media, the insulin production profile can be obtained, providing a deeper understanding of the system while minimizing the handling of the particles. All assays which require the collection of media, such as static GTT or the MTS metabolic assay, should be performed in Transwell plates (Corning) to facilitate quick particle/media separation with minimal particle handling.

The co-incubation of islets and fibers in the alginate suspension should be investigated to increase the likelihood of islet-fiber adhesion in the liquid phase, especially if the electrostatic system is to be used, as there is no emulsion-induced centralization and packing to encourage islet-fiber contact. Instances of islet-fiber adhesion were observed following an hour of co-incubation.

The electrostatic-mediated dropwise particle production method must be further optimized for islet viability, particle sphericity, and size distribution. Factors to be investigated include the diameter of the syringe, the fiber concentration density, fiber length, and alginate concentration. As the fiber concentration density increases, the concentration of the alginate matrix may have to be increased to maintain particle cohesion.

The emulsion system should also be developed further. Preliminary investigations indicated that fiber-laden particles produced from the shortest emulsion times were the smallest and most homogenous in composition, as core coalescence was minimized. However, a high concentration of fine, short fibers is necessitated to produce small, homogenous, discrete particles with 
sufficient fiber density. As the emulsion time is shortened, the risk of producing a small number of macrospheres $(>1 \mathrm{~mm})$ increases, which, while small in number, wastes a considerable volume of fibers and islets.

Following the optimization of the particle production method, the next stage in the research will be the surface modification of the fibers. Gelatin was selected for its physical and chemical properties, with some evidence that it would restore the islet-ECM connections lost during extraction (Del Guerra, 2001). However, the intent was always to build on the extensive pool of literature and incorporate specific proteins that are known to produce additional improvements in islet viability and insulin secretion, such as collagen IV and laminin (Weber, 2008).

The system is designed in such a way that a large supply of fibers can be produced and characterized, then modified in individual batches. This batch modification of the gelatin substrate is economical, as the bulk is composed of a commodity protein which can be highly crosslinked by genipin to maintain mechanical strength, while preserving carboxyl groups for EDC-NHS surface modification. The surface-modified batches can be combined in different ratios, allowing for a full factorial study without the issue of confounding the true effect of the surface modification with a change in the available protein surface area, the physical properties of the fiber, or the integrity of the particle.

The recombination of fibers from different ECM-modified batches (eg. 3 parts laminin fibers and 1 part collagen IV fibers) would have to be compared to a single batch of fibers modified with the same composition of ECM components ( $75 \mathrm{~mol} \%$ laminin and $25 \mathrm{~mol} \%$ collagen IV) to assess how the synergistic combination of laminin and collagen IV can be reproducibly achieved. Immunohistochemical staining for collagen IV and laminin would be required to assess the true 
surface composition, as the different components would have different adsorption affinities and EDC-NHS bonding kinetics.

Growth hormones, anti-inflammatory drugs and other pharmacological aids such as IGF II or genipin could be incorporated into the particles in three ways. The protein fibers could be rehydrated in a concentrated stock solution, allowing the compound to absorb into the fibers, acting as a depot within the particles. Alternatively, micro- and nanocapsules could be coencapsulated within the particles to provide controlled release of the compound. Finally, microcapsules containing chemical or biological sources of the compound could be incubated alongside the islet capsules.

As the fibers are contained within a standard alginate matrix, the particle surfaces can be modified with any method developed for alginate capsules, whether it is a simple poly-electrolyte layer or a more complex membrane to deter protein deposition and fibrotic overgrowth. However, the presence of gelatin at the particle surface, more of an issue with the electrostatic system than the emulsion system, may interfere with the consistency of ionically-formed membranes, unless the particles are re-coated first in a thin layer of pure alginate.

Beyond immunoisolation, the system developed here can be used for tissue engineering applications as well. Forming a covalent or poly-electrolyte membrane around a fiber-rich particle would allow the alginate core to be liquefied by chelation. Aggregates of stem cells, hepatocytes, islet-like cells or other adhesion-dependent proliferating cell lines could be cultured on the fibrous matrix within the capsule, filling the voids left between the fibers by the liquefied alginate. Upon attainment of sufficient growth, the membranes could be dissolved, or the capsules cultured in vascular endothelial cells to provide an anti-thrombogenic module for 3D printing or modular tissue engineering, such as the process developed by McGuigan and Sefton (2007). 
The opportunities for modification and optimization of the system outlined for the first time within this preliminary study are numerous, as it allows the implementation of a wealth of surface modifications developed for macroscopic geometries, but with the benefits of microparticle immunoisolation. 


\section{References}

Ahearne M, Yang Y, Then KY, Liu KK. Non-destructive mechanical characterisation of UVA/riboflavin crosslinked collagen hydrogels. Br J Ophthalmol. 2008; 92: 268-271.

Amory B, Mourmeaux JL, Remacle C. In vitro cytodifferentiation of perinatal rat islet cells within a tridimensional matrix of collagen. In Vitro Cell Dev Biol. 1988; 24: 91.

Armanet M, Wojtusciszyn A, Morel P, Parnaud G, Rousselle P, Sinigaglia C, Berney T, Bosco D. Regulated laminin-332 epression in human islets of Langerhans. FASEB. 2009; 23: 40464055.

Atkinson, MA. Thirty years of investigating the autoimmune basis for type 1 diabetes: why can't we prevent or reverse this disease? Diabetes. 2005; 54(5): 1253-1263.

Avery NC, Bailey AJ. Restraining cross-links responsible for the mechanical properties of collagen fibers: natural and artificial. In Collagen: Structure and Mechanics, Fratzl P (Ed). Springer: Potsdam. 2008; pp. 81-110.

Badylak SF, Tullius R, Kokini K, Shelbourne KD, Klootwyk T, Voytik SL, Kraine MR, Simmons C. The use of xenogeneic small intestinal submucosa as a biomaterial for Achilles tendon repair in a dog model. J Biomed Mater Res 1995;29(8):977-85.

Bailey AJ. Effects of ionizing radiation on connective tissue components. Int. Rev. Connect. Tissue Res. 1968; 4: 233-281.

Bank HL. Rapid assessment of islet viability with acridine orange and propidium iodide. In Vitro Cell \& Devel Biol. 1988; 24(4): 266-273.

Bara H, Sambinas A. Insulin-secreting L-cells for the treatment of insulin-dependent diabetes. Biochemical and Biophysical Research Communications. 2008; 37(1): 39-43.

Bara H, Sambanis A. Development and characterization of a tissue engineered pancreatic substitute based on recombinant intestinal endocrine 1-cells. Biotech and Bioeng. 2009; 103(4): 828-834.

Beattie GM, Montgomery AM, Lopez AD, Hao E, Perez B, Just ML, Lakey JR, Hart ME, Hayek A. A novel approach to increase human islet cell mass while preserving beta-cell function. Diabetes. 2002; 51: 3435 .

Berridge MV, Tan AS. Characterization of the cellular reduction of 3-(4,5-dimethylthiazol-2-yl)2,5-diphenyltetrazolium bromide (MTT): Subcellular localization, substrate dependence, and involvement of mitochondrial electron transport in MTT reduction. Arch Biochem Biophys. 1993; 303: 474-82.

Bertoni F, Barbani N, Giusti P, Ciardelli G. Transglutaminase reactivity with gelatin: perspective applications in tissue engineering. Biotechnol Lett. 2006; 28: 697-702.

Bigi A, Cojazzi G, Panzavolta S, Roveri N, Rubini K. Stabilization of gelatin films by crosslinking with genipin. Biomaterials. 2002; 23: 4827-4832. 
Bosco D, Meda P. Reconstructing islet function in vitro. Advances in experimental medicine and biology. 1997; 426: 285-298.

Brandenburg D. History and diagnostic significance of C-peptide. Exp Diabetes Res. 2008; 576862.

Brendel MD, Kong SS, Alejandro R, Mintz DH, Improved functional survival of human islets of Langerhans in three-dimensional matrix culture. Cell Transplant. 1994; 3: 427.

Brodsky B, Persikov AV. Molecular structure of the collagen triple helix. Adv Protein Chem. 2005; 70: 301-339.

Calafiore R, Basta G, Luca G, Lemmi A, Montanucci MP, Calabrese G, Racanicchi L, Manusco F, Brunetti P. Microencapsulated pancreatic islet allografts into nonimmuno-suppressed patients with type 1 diabetes. Emerging Treatments and Tech. 2006A; 29(1): 137-138.

Calafiore R, Basta G, Luca G, Lemmi A, Montanucci MP, Racanicchi L, Manusco F, Brunetti P. Standard technical procedures for microencapsulation of human islets for graft into nonimmunosuppressed patients with type 1 diabetes mellitus. Transplantation Proceedings. 2006B; 38: 1156-1157.

CDA, Canadian Diabetes Association. The prevalence and cost of diabetes. April 2008. http://www.diabetes.ca/Files/prevalence-and-costs.pdf Accessed May 24, 2011.

CDA, Canadian Diabetes Association. Diabetes: Canada at the tipping point. April 2011. http://www.diabetes.ca/documents/get-involved/WEB Eng.CDA Report .pdf Accessed May $24,2011$.

Chang Y, Tsai CC, Liang HC, Sung HW. In vivo evaluation of cellular and acellular bovine pericardia fixed with a naturally occurring crosslinking agent (genepin). Biomaterials. 2002; 23: $2447-2457$.

Chau DYS, Collighan RJ, Elisabetta AM,Addy VL, Griffin M. The cellular response to transglutaminase-cross-linked collagen. Biomaterials. 2005; 26: 6518-6529.

Chen RN, Ho HO, Sheu MT. Characterization of collagen matrices crosslinked using microbial transglutaminase. Biomaterials. 2005; 26: 4229-4235.

Chen T, Small DA, McDermott MK, Bentley WE, Payne GF. Enzymatic methods for in situ cell entrapment and cell release. Biomacromolecules. 2003; 4: 1558-1563.

Cheng JYC, Rughunath M, Whitelock J, Poole-Warren L. Matrix components and scaffolds for sustained islet function. Tissue Engineering: Part B. 2011; 17(4): 235-247.

Chun S, Huang Y, Xiw WJ. Adhesive growth of pancreatic islet cells on a PGA fibrous scaffold. Trans. Proc. 2008; 40: 1658-1663.

Chin GS, Lee S, Hsu M, Jiu W, Kim, WJH, Levinson H, Longaker MT. Discoidin domain receptors and their ligand, collagen, are temporarily regulated in fetal rat fibroblasts in vitro. Plast Reconstr Surg. 2001; 107: 769-776. 
Ciardelli G, Gentile P, Chiono V, Mattioli-Belmonte M, Vozzi G, Barbani N, Giusti P. Enzymatically crosslinked porous composite matrices for bone tissue regeneration. J Biomed Mater Res Part A. 2010; 92(1): 137-151.

CITR, Collaborative Islet Transplant Registry. Fifth Annual Report. Sept 2008. http://www.citregistry.org.

CITR, Collaborative Islet Transplant Registry. Sixth Annual Report. Nov 2009. http://www.citregistry.org.

Clausen AE, Bernkop-Schnürch A. In vitro evaluation of the permeation enhancing effect of thiolated polycarbophil. J. Pharm.Sci. 2000; 89: 1253-1261.

Colella RM, Bonner-Weir S, Braunstein LP, Schwalke M, Weir GC. Pancreatic islets of variable size - insulin secretion and glucose utilization. Life Sciences. 1985; 37 (11); 1059-1065.

Covington AD. Modern tanning chemistry. Chem. Soc. Rev. 1997; 26: 111-126.

Crescenzi V, Francescangeli A, Taglienti A. New gelatin-based hydrogels via enzymatic networking. Biomacromolecules. 2002; 3: 1384-1391.

Damink LHHO, Dijkstra PJ, vanLuyn MJA, Vanwachem PB, Nieuwenhuis P, Feijen J. Crosslinking of dermal sheep collagen using hexamethylene diisocyanate. J Mater Sci Mater MED. 1995; 6: 429-434.

Daoud J, Petropavlovskaia M, Rosenberg L, Tabrizian M. The effect of extracellular matrix components on the preservation of human islet function in vitro. Biomaterials. 2010;31: $1676-1682$.

Daoud JT, Petropavlovskaia MS, Patapas JM, Degrandpré CE, DiRaddo RW, Rosenberg L, Tabrizian M. Long-term in vitro human pancreatic islet culture using three-dimensional microfabricated scaffolds. Biomaterials. 2011; 32: 1536-1542.

DCCT, The Diabetes Control and Complications Trial Research Group. The effect of intensive treatment of diabetes on the development and progression of long-term complications in insulin-dependent diabetes mellitus. N Eng1 J Med. 1993; 329: 977-986.

de Carlo E, Baiguerra S, Conconi MT, Vigolo S, Grandi C, Lora S, Martini C, Maffei P, Tamagno G, Vettor R, Sicolo N, Parnigotto PP. Pancreatic acellular matrix supports islet survival and function in a synthetic tubular device: In vitro and in vivo studies. Int $\mathrm{J}$ Mol Med. 2010; 25: 195-202.

de Vos P, Hamel AF, Tatarkiewicz K. Considerations for successful transplantation of encapsulated pancreatic islets.Diabetologia. 2002; 45:159-173.

de Vos P, Faas MM, Strand B, Calafiore R. Alginate-based microcapsules for immunoisolation of pancreatic islets. Biomaterials. 2006A; 27: 5603-5617.

de Vos P, Andersson A, Tam SK, Fass M, Hallé JP. Advances and barriers in mammalian cell encapsulation for treatment of diabetes. Immun Endoc and Metab Agents in Med Chem. 2006B; 6(2): 139-153. 
de Vos P, Faas MM, Strand B, Calafiore R. Alginate-based microcapsules for immunoisolation of pancreatic islets. Biomaterials. 2006C; 27: 5603-5617.

de Vos P, Bucko M, Gemeiner P, Navratil M, Svitel J, Faas M, Strand BL, Skjak-Braek G, Morch YA, Vikartovska A, Lacik I, Kollarikova G, Orive G, Poncelet D, Pedraz JL, AnsorgeSchumacher MB. Multiscale requirements for bioencapsulation in medicine and biotechnology. Biomaterials. 2009A; 30:2559-2570.

de Vos P, de Haan BJ, Faas MM. Different approaches for immunoisolation. In The bioartificial pancreas and other biohybrid techniques. Hallé JP, de Vos P, Rosenberg L (eds). Transworld Research Network, Kerala, India. 2009: pp 27- 36.

Del Guerra, Bracci C, Nilsson K. Entrapment of dispersed pancreatic islet cells in CultiSpher-S macroporous gelatin microcarriers. Biotech. And Bioeng. 2001; 75(6): 741-744.

Duan X, Sheardown H. Dendrimer crosslinked collagen as a corneal tissue engineering scaffold: mechanical properties and corneal epithelial cell interactions. Biomaterials. 2006; 27: 46084617.

Duckworth WC, Bennett RG, Hamel FG. Insulin Degradation: Progress and Potential. Endocrine Reviews. 1998; 19(5): 608-624.

Dugas V, Beauchamp C, Hallé JP, Lesage S. Protection by microencapsulation from antigen recognition, immune cells, antibodies, and compliment components. The bioartificial pancreas and other biohybrid techniques. Hallé JP, de Vos P, Rosenberg L (eds). Transworld Research Network, Kerala, India. 2009: pp 261-277.

Eich T, Eriksson O, Sundin A. Positron emission topography: a real time tool to quantify early islet engraftment in a preclinical large animal model. Transplantation. 2007; 84: 893-898.

Elliot RB, Escobar L, Tan PLJ, Muzina M, Zwain S, Buchanan C. Live encapsulated porcine islets from a type 1 diabetic patient $9,5 \mathrm{yr}$ after xenotransplantation. Xenotransplantation. 2007; 14: 157-161.

Fakirov S. Gelatin and gelatin-based biodegradable composites. In Handbook of Engineering Biopolymers: Homopolymers, Blends, and Composites. Hanser: Munich. 2007; pp 423.

Flakoll PJ, Jensen MD, Cherrington AD. Physiologic Action of Insulin. Diabetes Mellitus: A Fundamental and Clinical Text. 2004 (11).

Fukae R, Midorikawa T. Preparation of gelatin fiber by gel spinning and its mechanical properties. J App Poly Sci. 2008; 110(6): 4011-4015.

Gimi B, Kwon J, Liu L, Su Y, Nemani K, Trivedi K, Cui Y, Vachha B, Mason R, Hu W, Lee JB. Cell encapsulation and oxygenation in nanoporous microcontainers. Biomed Microdevices. 2009; 11: 1205-1212.

Glowacki J. Mizuno S. Collagen scaffolds for tissue engineering. Biopolymers. 2007; 89(5): 338342.

Gotoh M, Maki T, Kiyoizumi T, Satomi S, Monaco AP. An improved method for isolation of mouse pancreatic islets. Transplantation. 1985; 40:437-438. 
Griffin M, Casadio R, Bergamini CM. Transglutaminases: nature's biological glues. Biochem J. 2002; 368: 377-396.

Guidry C, Grinnell F. Studies on the mechanism of hydrated collagen gel reorganization by human skin fibroblasts. J. Cell. Sci. 1985; 79: 67-81.

Guilak F, Butler DL, Goldstein SA, Mooney DJ. Functional Tissue Engineering. SpringerVerlag: New York. 2003; pp. 426.

Hallé JP, de Vos P. The need for new therapeutic approaches and the bioartificial endocrine pancreas. In The bioartificial pancreas and other biohybrid techniques. Hallé JP, de Vos P, Rosenberg L (eds). Transworld Research Network, Kerala, India. 2009: pp1-26.

Hamamoto Y, Fujimoto S, Inada A, Takehiro M, Nabe K, Shimono D, Kajikawa M, Fujita J, Yamada Y, Seino Y. Beneficial effect of pretreatment of islets with fibronectin on glucose tolerance after islet transplantation. Horm Metab Res. 2003; 35: 460-465.

Harkness RD. Biological functions of collagen. Biol. Rev. 1961; 36: 399-463.

Hellman B. Pulsatility of insulin release - a clinically important phenomenon. Upsula J Med Sci. 2009; 114: 193-205.

Hiscox AM, Stone AL, Limesand S, Hoying JB, Williams SK. An islet-stabilizing implant constructed using a preformed vasculature. Tissue Engineering: Part A. 2008; 14(3): 433-440.

Hoesli C, Raghuram K, Donald C, Kiang R, Kieffer TJ, Piret JM. Pancreatic cell encapsulation by alginate emulsion and internal gelation. XVIIthe International Conference on Bioencapsulation, Groningen, Netherlands. Sept 2009. Oral 10-4.

Hoesli CA, Raghuram K, Kiang RLJ, Mocinecova D, Hu X, Johnson JD, Lacik I, Kieffer TJ, Piret JM. Pancreatic cell immobilization in alginate beads produced by emulsion and internal gelation. Biotech Bioeng. 2011; 108: 424-434.

Hori Y, Gu X, Xie X, Kim SK. Differentiation of insulin-producing cells from human neural progenitor cells. PLoS Med. 2005; 2: 347.

Horwitz EM, Prockop DJ, Fitzpatrick LA, Koo WWk, Gordon PL, Neel M, Sussman M, Orchard P, Marx JC, Pyeritz RE, Brenner MK. Transplantability and therapeutic effects of bone marrow-derived mesenchymal cells in children with osteogenesis imperfecta. Nature Medicine. 1999; 5: 309-313.

Hou Y, Song C, Xie WJ, Wei Z, Huang RP, Liu W, Zhang ZL, Shi YB. Excellent effect of threedimensional culture condition on pancreatic islets. Diabetes Research and Clinical Practice. 2009; 86: 11-15.

Hu X, Ma L, Wang C, Gau C. Gelatin hydrogel prepared by photo-initiated polymerization and loaded with TGF- $\beta 1$ for cartilage tissue engineering. Macromolecular Bioscience. 2009; 9: 1194-1201.

Huang D, Chang TR, Aggarwal A, Lee RC, Ehrlich HP. Mechanisms and dynamics of mechanical strengthening in ligament-equivalent fibroblast-populated collagen matrices. Ann. Biomed. Eng. 1993; 21: 289-305. 
Hulmes DJS. Building collagen molecules, fibrils, and suprafibrillar structures. J Struct Biol. 2002; 137: 2-10.

Hulmes DJS. Collagen diversity, synthesis and assembly. In Collagen: Structure and Mechanics, Fratzl P (Ed). Springer: Potsdam. 2008; pp. 81-110.

Ilieva A, Yuan S, Wang RN, Agapitos D, Hill DJ, Rosenberg L. Pancreatic islet cell survival following islet isolation: the role of cellular interactions in the pancreas. J Endocronol. 1999; 161: 357-364.

IPTR, International Pancreas Transplant Registry. May 1, 2009. http://www.iptr.umn.edu/ . Accessed Nov 20, 2009.

Irving Rodgers HF, Ziolkowski AF, Parish CR, Sado Y, Ninomiya Y, Simeonovic CJ, Rodgers RJ. Molecular composition of the peri-islet basement membrane in NOD mice: a barrier against destructive insulitis. Diabetologia. 2008; 51: 1680-1688.

Jansson L, Carlsson PO. Graft vascular function after transplantation of pancreatic islets. Diabetologia. 2002; 45:749.

Jiang FX, Naselli G, Harrison LC. Distinct distribution of laminin and its integrin receptors in the pancreas. J Histochem Cytochem. 2002; 50: 1625-1632.

Jourdan G, Dusseault J, Benhamou PY, Rosenberg L, Hallé JP. Co-encapsulation of bioengineered IGF-II producing cells and pancreatic islets. Gene Therapy. 2011; 18: 539-545.

Kadler KE, Holmes DF, Trotter JA, Chapman JA. Collagen fibril formation. Biochem J. 1996; 316: $1-11$.

Kadler KE, Baldock C, Bella J, Boot-Handford RP. Collagens at a glance. J Cell Sci. 2007; 120: $1955-1958$.

Kaido T, Yebra M, Cirulli V, Montgomery AM. Regulation of human beta-cell adhesion, motility, and insulin secretion by collagen IV and its receptor alphalbeta1. J Biol Chem. 2004; 279: 53762-9.

Kashiwagi T, Yokoyama KI, Ishikawa K, Ono K, Ejima D, Matsui H, Suzuki EI. Crystal structure of microbial transglutaminase from Streptoverticillium mobaraense. J. Biol. Chem. 2002; 277: 44252-44260.

Kato Y, Uchida K, Kawakishi S. Aggregation of collagen exposed to uva in the presence of riboflavin: a plausible role of tyrosine modification. Photochem Photobiol. 1994; 59: 343349.

Kato Y, Nishikawa T, Kawakishi S. Formation of protein-bound 3,4 dihydroxyphenylalanine in collagen types I and IV exposed to ultraviolet light. Photochem. Photobiol. 1995; 61: 367372.

Kawazoe N, Lin X, Tateishi T, Chen G. Three-dimensional cultures of rat pancreatic RIN-5F cells in porous PLGA-collagen hybrid scaffolds. J. Bioact and Compat. Poly. 2009; 24: 25-42.

Khor E. Methods for the treatment of collagenous tissues for bioprostheses. Biomaterials. 1997; 18: 95-105. 
Kobayashi N. Bioartificial pancreas for the treatment of diabetes. Cell Transplantation. 2008; 17: $11-17$.

Kodama S, Kuhtreiber W, Fujimura S, Dale EA, Faustman DL. Islet regeneration during the reversal of auto immune diabetes in NOD mice. Science. 2003; 302: 1223.

Lacy PE, Kostianovsky M. Method for the isolation of intact islets of Langerhans from the rat pancreas. Diabetes. 1967; 16:35-39.

Landers R, Pfister A, Hubner U, John H, Schmelzeisen R, Mulhaupt R. Fabrication of soft tissue engineering scaffolds by means of rapid prototyping techniques. 2002; 37(15): 3107-3116.

Langlois G, Dusseault J, Bilodeau S, Tam SK, Magassouba D, Hallé JP. Direct effect of alginate purification on the survival of islets immobilized in alginate-based microcapsules. Acta Biomaterialia. 2009; 5: 3433-3440.

Latif ZA, Noel J, Alejandro R. A simple method of staining fresh and cultured islets. Transplantation.1988; 45(4): 827-830.

Lau TT, Wang C, Png SW, Su K, Wang DA. Genipin-crosslinked microcarriers mediating hepatocellular aggregates formation and functionalities. J Biomed Mat Res A. 2011; 96 (1): 204-211.

Leblond FA, Simard G, Henley N, Rocheleau B, Huet PM, Hallé JP. Studies on smaller (similar to $315 \mu \mathrm{m})$ microcapsules: IV feasibility and safety of intrahepatic implantations of small alginate poly-L-lysine microcapsules. Cell Transplant. 1999; 8(3): 327-337.

Lechner A, Habener JF. Stem/progenitor cells derived from adult tissues: potential for the treatment of diabetes mellitus. Amer. J. of Physio. - Endocr. and Metab. 2003; 284(2):E259E266.

Lee CH, Singla A, Lee Y. Biomedical applications of collagen. Int J Pharm. 2001; 221: 1-22.

Liang HC, Chang WH, Lin KJ, Sung HW. Genipin-crosslinked gelatin microspheres as a drug carrier for intramuscular administration: In vitro and in vivo studies. J Biomed Mat Res Part A. $2002 ; 65 \mathrm{~A}(2): 271-282$.

Lim F, Sun AM. Microencapsulated islets as bioartificial endocrine pancreas. Science. 1980; 210: 908-910.

Lim DJ, Antipenko SV, Anderson JM, Jimes KF, Viera L, Stephen BR, Bryant SMJ, Yancey BD, Hughes KJ, Cui W, Thompson JA, Corbett JA, Jun HW. Enhanced rat islet function and survival in vitro using a biomimetic self-assembled nanomatrix gel. Tissue Eng: Part A. 2011; 17(3): 399-406.

Liu Y, Petreaca M, Martins-Green M. Cell and molecular mechanisms of insulin-induced angiogenesis. J. Cellular and Molecular Medicine. 2008; 12(6): 1-13.

Lucas-Clerc C, Massart C, Campion JP, Launois B, Nicol M. Long-term culture of human pancreatic islets in an extracellular matrix: Morphological and metabolic effects. Mol Cell Endocrinol. 1993; 94: 9-20. 
Lumelsky N, Blondel O, Laeng P, Velasco I, Ravin R, McKay R. Differentiation of embryonic stem cells to insulin-secreting structures similar to pancreatic islets. Science. 2001; 292: 13891394.

Lutolf MP, Hubbell JA. Synthetic biomaterials as instructive extracellular microenviron-ments for morphogenesis in tissue engineering. Nat Biotech. 2005; 23: 47-55.

Mallet AG, Korbutt GS. Alginate modification improves long-term survival and function of transplanted encapsulated islets. Tissue Engineering: Part A. 2009; 15(6): 1301-1309.

Martini FH, Timmons MJ, Tallistch RB. Human Anatomy: Sixth Edition. Pearson Education Inc. Toronto. 2008: 518-519, 685-686.

Martins-Green M. The dynamics of cell-ECM interactions with implications for tissue engineering, in Principles of Tissue Engineering. Lanza R, Langer R, Chick W (Eds). RG Landes Company. 1997. pp. 23-46.

Matsuda S, Iwata H, Se N, Ikada Y. Bioadhesion of gelatin films crosslinked with glutaraldehyde. J Biomed Mater Res. 1999; 45(1): 20-27.

McDermott MK, Chen T, Milliams CM, Markley KM, Payne GF. Mechanical properties of biomimetic tissue adhesive based on the microbial transglutaminase-catalyzed crosslinking of gelatin. Biomacromolecules. 2004; 5: 1270-1279.

McGuigan AP, Sefton MV. Modular tissue engineering: fabrication of a gelatin-based construct. J Tissue Eng Regen Med. 2007; 1: 136-145.

Ménard M, Dusseault J, Langlois G, Baille WE, Tam SK, Yahia LH, Zhu XX, Hallé JP. Role of protein contaminants in the immunogenicity of alginates. J Biomed Mat Res: Part B. 2010; 93(2):333 - 340 .

Mercodia. Mercodia Insulin ELISA. Article 32-4143. April 2010. www.mercodia.se.

Miles CA, Sionkowska A, Hulin S, Sims TJ, Avery NC, Bailey AJ. Identification of an intermediate state in the helix coil degradation of collagen by UV light. J. Biol. Chem. 2000; 275: 33014-33020.

Miles CA, Avery NC, Rodin V and Bailey AJ. The increase in denaturation temperature following cross-linking is caused by dehydration of the fibres. J. Mol. Biol. 2005; 346: 551556.

Miyazaki JI, Araki K, Yamato E, Ikegami H, Asano T, Shibasaki Y, Oka Y, Yamamura KI. Establishment of a pancreatic $\beta$-cell line that retains glucose-inducible insulin secretion: special reference to expression of glucose transporter isoforms. Endocrinology. 1990: 127: 126-132.

Mladenovska K, Cruaud O, Richomme P, Belamie E, Raicki RS, Venier-Julienne MC, Popovski E, Benoit JP, Goracinova K. 5-ASA loaded chitosan-Ca-alginate microparticles: preparation and physicochemical characterization. Int. J. Pharma. 2007; 345: 59-69.

Monaco AP, Maki T, Ozato H, Carretta M, Sullivan SJ, Borland KM, Mahoney MD, Chick WL, Muller TE, Wolfrum J, Solomon B. Transplantation of islet allografts and xenografts in totally 
pancreatectomized diabetic dogs using the hybrid artificial pancreas. Annals of Surgery. 1991; 214(3): 339-362.

Mueller-Kliester WE, Sutherland RM. Influence of convection in the growth medium on oxygen tensions in multicellular tumor spheroids. Cancer Research. 1982; 42(1): 237-242.

Muthyala S, Raj VRR, Mohanty M, Mohanan PV, Nair PD. The reversal of diabetes in rat model using mouse insulin producing cells - A combination approach of tissue engineering and macroencapsulation. Acta Biomaterialia. 2011; 7: 2153-2162.

Nakagawa S, Pawelek P, Grinnell F. Long-term culture of fibroblasts in contracted collagen gels: effects on cell growth and biosynthetic activity. J. Invest. Dermatol. 1989; 93: 792-798.

Nguyen TH, Lee BT. Fabrication and characterization of cross-linked gelatin electro-spun nanofibers. J Biomat Sci and Eng. 2010; 3: 1117-1124.

Nolan AL, O'Dowd JF. The measurement of insulin secretion from isolated rodent islets of Langerhans. In Type 2 Diabetes, Methods in Molecular Biology, v560. Stocker C (ed). Humana Press, New York, USA. 2009; 43-51.

O'Sullivan ES, Johnson AS, Omer A, Hollister-Lock J, Bonner-Weir S, Colton CK, Weir GC. Rat islet cell aggregates are superior to islets for transplantation in microcapsules. Diabetologia. 2010; 53: 937-945.

Olsen D, Yang C, Bodo M, Chang R, Leigh S, Baez J, Carmichael D, Perala M, Hamalainen EJ, Jervinen M, Polarek J. Recombinant collagen and gelatin for drug delivery. Adv Drug Delivery Reviews. 2003; 55: 1547-1567.

Omer A, Duvivier-Kali V, Fernandes J. Long-term normoglycemia in rats receiving transplants with encapsulated islets. Transplantation. 2005; 79(1): 52-58.

Orive G, Hernandez RM, Gascon AR, Calafiore R, Chang TMS, de Vos P, Hortelano G, Hunkeler D, Lacik I, Pedraz JL. History, challenges and perspectives of cell microencapsulation. Trends in Biotechnology. 2004; 22(2): 87-92.

Perez-Basterrechea M, Briones RM, Alvarez-Viejo M, Garcia-Perez E, Esteban MM, Garcia V, Obaya A, Barneo L, Meana A, Otero J. Plasma-fibroblast gel as scaffold for islet transplantation. Tissue Engineering: Part A. 2009; 15(3): 569-577.

Petite H, Duval LL, Frei V, Abdulmalak N, Sigotluizard MF, Herbage D. Cytocompatibility of calf pericardium treated by glutaraldehyde and by acyl azide methods in an organotypic culture model. Biomaterials. 1995; 16: 1003-1008.

Poncelet AJ, Denis D, Gianello P. Cellular xenotransplantation. Curr Opin Org Trans. 2009; 14:168-174.

Poncelet D, Lencki R, Beaulie C, Hallé JP, Neufeld RJ, Fournier A. Production of alginate beads by emulsification/internal gelation. I. Methodology. Appl Microbiol Biotechnol. 1992; 38: 3945. 
Poncelet D, Poncelet De Smet B, Beaulieu C, Huguet ML, Fournier A, Neufeld RJ. Production of alginate beads by emulsion/internal gelation. II. Physicochemistry. Appl Microbiol Biotechnol. 1995; 43: 644-650.

Poncelet D, Tam SK. Microencapsulation technologies for a bioartifical endocrine pancreas. In The bioartificial pancreas and other biohybrid techniques. Hallé JP, de Vos P, Rosenberg L (eds). Transworld Research Network, Kerala, India. 2009: pp 37-50.

Ricard-Blum S, Ruggiero F, van der Rest M. The collagen superfamily. Top Curr Chem. 2005; 247: 35-84.

Robitaille R, Dusseault J, Henley N, Rosenberg L, Hallé JP. Insulin-like growth factor II allows prolonged blood glucose normalization with a reduced islet cell mass transplantation. Endocrinology. 2003; 144(7): 3037-3045.

Robitaille R, Hallé JP. Characteristion and evaluation of the properties and functions of the microcapsules. In The bioartificial pancreas and other biohybrid techniques. Hallé JP, de Vos P, Rosenberg L (eds). Transworld Research Network, Kerala, India. 2009: pp 67-85.

Roche Diagnostics GmbH. Liberase RI. http://www.roche-applied-science.com. August 2008.

Rosenberg L, Wang RN, Paraskevas S, Maysinger D. Structural and functional changes resulting from islet isolation lead to islet cell death. Surgery. 1999; 126(2): 393-398.

Rosso F, Marino G, Giordano A, Barbarisi M, Parmeggiani D, Barbarisi A. Smart materials as scaffolds for tissue engineering. J Cell Phys. 2005; 203: 465-470.

Ruggiero F, Koch M. Making recombinant extracellular matrix proteins. Methods. 2008; 45: 7585.

Salvay DM, Rives CB, Zhang X, Chen F, Kaufman DB, Lowe WL, Shea LD. Extracellular matrix protein-coated scaffolds promote the reversal of diabetes after extrahepatic islet transplantation. Transplantation. 2008; 85(10): 1456-1464.

Sanborn TJ, Messersmith PB, Barron AE. In situ crosslinking of a biomimetic peptide-PEG hydrogel via thermally triggered activation of factor XIII. Biomaterials. 2002; 23: 2703-2710.

Sandler S, Eizirik DL, Sternesjo J, Welsh N. Role cytokines in regulation of pancreatic b-cell function.Biochem.Soc.Trans. 1994; 22:26-30.

Sapir T, Shternhall K, Meivar-Levy I, Blumenfeld T, Cohen H, Skutelsky E, Eventov-Friedman S, Barshack I, Goldberg I, Pri-Chen S, Ben-Dor L, Polak-Charcon S, Karasik A, Shimon I, Ferber S. Cell-replacement therapy for diabetes: generating functional insulin-producing tissue from adult human liver cells. Proc Natl Acad Sci. 2005; 102: 7964-7969.

Scharp DW, Lacy PE, Santiago JV, McCullough CS, Weide LG, Falqui L, Marchetti P, Gingerich RL, Jaffe AS, Cryer PE. Insulin independence after islet transplantation into type I diabetic patient. Diabetes. 1990; 39: 515-518.

Segev H, Fishman B, Ziskind A, Shulman M, Itskovitz-Eldor J. Differentiation of human embryonic stem cells into insulin producing clusters. Stem Cells. 2004; 22: 265-274. 
Serban MA, Prestwich GD. Modular extracellular matrices: Solutions for the puzzle. Methods. 2008; 45: 93-98.

Shapiro AMJ, Lakey JRT, Ryan EA, Korbutt GS, Toth E, Warnock GL, Kneteman NM, Rajotte $\mathrm{RV}$. Islet transplantation in seven patients with type 1 diabetes mellitus using a glucocorticoidfree immunosuppressive regimen. NEJM. 2000; 343(4): 230-238.

Shapiro AMJ, Gallant HL, Hao EG, Lakey JRT, McCready T, Rajotte RV, Yatscoff RW, Kneteman NM. The portal immunosuppressive storm - relevance to islet transplantation?. Therapeutic Drug Monitoring. 2005; 27(1):35-37.

Shapiro AMJ, Ricordi C, Hering BJ, Auchincloss H, Lindblad R, Robertson P, Secchi A, Brendel $\mathrm{MD}$, Berney T, Brennan DC, Cagliero E et al. International trial of the Edmonton protocol for islet transplantation. NEJM. 2006; 355(13): 1318-1330.

Sheu MT, Huang JC, Yeh GC, Ho HO. Characterization of collagen gel solutions and collagen matrics for cell culture. Biomaterials. 2001; 22: 1713-1719.

Sigma Aldrich. Product Information: Gelatin, CAS RN 9000-70-8. Nov 2008.

Sisson K, Zhang C, Farach-Carson MC, Chase DB, Rabolt J. Evaluation of cross-linking methods for electrospun gelatin on cell growth and viability. Biomacromolecules. 2009; 10(7): 16761680 .

Skarulis MC, Hirshberg B. Hypoglycemia in the Adult. In Diabetes Mellitus: A Fundamental and Clinical Text. LeRoith D, Taylor SI, Olefsky JM (eds). Lippincott Williams \& Wilkins, Philadelphia, USA. 2004: pp 1427-1438.

Slusarewicz P, Zhu K, Hedman T. Kinetic characterization and comparison of various protein crosslinking reagents for matrix modification. J Mater Sci: Mater Med. 2010; 21: 1175-1181.

Smidsrød O, Skjåk-Braek. Alginate as immobilization matrix for cells. Trends Biotechnol. 1990; 8: $71-78$.

Sollinger HW, Sasaki TM, D'Alessandro AM. Indications for enteric conversion after pancreas transplantation with bladder drainage. Surgery. 1992; 842-845.

Song JH, Kim HE, Kim HW. Production of electrospun gelatin nanofiber by water-based cosolvent approach. J Mater Sci: Mater Med. 2008; 19: 95-102.

Stegall MD, Dean PG, Sung R. The rationale for the new deceased donor pancreas allocation schema. Transplantation. 2007; 83: 1156-1161.

Stock UA, Vacanti JP. Tissue engineering: current state and prospects. Annu Rev Med. 2001; 52: 443-451.

Sung HW, Huang DM, Chang WH, Huang RN, Hsu JC. Evaluation of gelatin hydrogel crosslinked with various crosslinking agents as bioadhesives: In vitro study. J Biomed Mat Res. 1999; 46(4): 520-530.

Sung HW, Liang IL, Chen CN, Huang RN, Liang HF. Stability of a biological tissue fixed with a naturally occurring crosslinking agent (genipin). J Biomed Mat Res. 2001; 55(4): 538-546. 
Tatarkiewicz K, Hollister-Lock J, Quickel RR, Colton CK, Bonner Weir S, Weir GC. Reversal of hyperglycemia in mice after subcutaneous xenografts of encapsulated islets. Transplantation. 1999; 67:655-671.

Thomas FT, Contreras JL, Bilbao G, Ricordi C, Curiel D, Thomas JM. Anoikis, extracellular matrix, and apoptosis factors in isolated cell transplantation. Surgery. 1999; 126 (2): 299304.

Thomas EK, Nakamura M, Wienke D, Isacke CM, Pozzi A, Liang P. Endo180 binds to the Cterminal region of type I collagen. J. Biol. Chem. 2005; 280, 22596-22605.

Thu B, SkjakBraek G, Micali F, Vittur F, Rizzo R. 1997. The spatial distribution of calcium in alginate gel beads analysed by synchrotronradiation induced X-ray emission (SRIXE). Carbohydr Res 297(2):101-105.

Torres-Giner S, Gimeno-Alcaniz JV, Ocio MJ, Laragon JM. Comparative performance of electrospun collagen nanofibers cross-linked by means of different methods. Applied Materials and Interfaces. 2009; 1(1): 218-223.

Tranquillo RT. Self-organization of tissue-equivalents: the nature and role of contact guidance. Biochem. Soc. Symp. 1999; 65: 27-42.

Tuch BE, Keogh GW, Williams LJ, Wu W, Foster JL, Vaithilingam V, Philips R. Safety and viability of microencapsulated human islets transplanted into diabetic humans. Emerg Treatments and Tech. 2009; 32(10): 1887-1889.

van Deijnen JH, Hulstaert CE, Wolters GH, van Schilfgaarde R. Significance of the peri-insular extracellular matrix for islet isolation from the pancreas of rat, dog, pig, and man. Cell Tissue Res. 1992; 267:139-146.

Wang RN, Paraskevas S, Rosenberg L. Characterization of integrin expression in islets isolated from hamster, canine, porcine, and human pancreas. J Histochem Cytochem. 1999; 163(2): 181-190.

Wang X, Ye K. Three-dimensional differentiation of embryonic stem cells into islet-like insulinproducing clusters. Tissue Engineering: Part A. 2009; 15(8): 1941-1951.

Weber LM, Hayda KN, Anseth KS. Cell-matrix interactions improve $\beta$-cell survival and insulin secretion in three-dimensional culture. Tissue Engineering: Part A. 2008; 14(12): 1959-1967.

White SA, Shaw JA, Sutherland DER. Pancreas transplantation. The Lancet. 2009; 373: 18081817.

Williams BR, Gelman RA, Poppke DC, Piez KA . Collagen fibril formation: optimal in vitro conditions and preliminary kinetic results. J Biol Chem. 1978; 235: 6578-6585.

Williams ME, Lacson E, Teng M, Ofsthun N, Lazarus JM. Hemodialyzed type I and type II diabetic patients in the US: characteristics, glycemic control, and survival. Kidney Int. 2006; 70: 1503-1509.

Williams PW. Notes on diabetes treated with extract and by grafts of sheep's pancreas. British Medical Journal. 1894; 2:1303-1304. 
Wollensak G, Spoerl E, Seiler T. Riboflavin/ultraviolet-A-induced collagen crosslinking for the treatment of keratoconus. Am J Ophthalmol. 2003; 135:620-627.

Wu LF, Wang NN, Liu YS, Wei X. Differentiation of Wharton's jelly primitive stromal cells into insulin-producing cells in comparison with bone marrow mesenchymal stem cells. Tissue Engineering: Part A. 2009; 15(10): 2865-2873.

$\mathrm{Xu} \mathrm{C}, \mathrm{Yu} \mathrm{Z}$, Inouye M, Brodsky B, Mirochnitchenko O. Expanding the family of collagen proteins: recombinant bacterial collagens of varying composition form triple-helices of similar stability. Biomacromolecules. 2010; 11: 348-356.

Yamamoto K, Yamamoto M. Cell adhesion receptors for native and denatured type I collagens and fibronectin in rabbit arterial smooth muscle cells in culture. Exp. Cell Res. 1994; 214, $258-263$.

Yang CY, Chiu CT, Chang YP, Wang YJ. Fabrication of porous gelatin microfibers using an aqueous wet spinning process. Artificial Cells, Blood Substitutes, and Biotechnology. 2009; 37:173-176.

Yao CH, Liu BS, Chang CJ, Hsu SH, Chen YS. Preparation of networks of gelatin and genipin as degradable biomaterials. Mat Chem Phys. 2004. 83: 204-208.

Zalzman M, Gupta S, Giri RK, Berkovich I, Sappal BS, Karnieli O, Zern MA, Fleischer N, Efrat S. Reversal of hyperglycemia in mice by using human expandable insulin producing cells differentiated from fetal liver progenitor cells. Proc Natl Acad Sci. 2003; 100: 7253-7258.

Zekorn T, Siebers U, Horcher A, Schnettler R, Klöck G, Bretzel RG, Zimmermann U, Federlin $\mathrm{K}$. Barium-alginate beads for immunoisolated transplantation of islets of Langerhans. Trans Proc. 1992; 24(3): 937-939.

Zhang CY, Parton LE, Ye CP, Krauss S, Shen R, Lin CT, Porco JA, Lowell BB. Genipin inhibits UCP2-mediated proton leak and acutely reverses obesity- and high glucose-induced $\beta$ cell dysfunction in isolated pancreatic islets. Cell Metabolism. 2006A; 3: 417-427.

Zhang YZ, Venugopal J, Hunag ZM, Lim CT, Ramakrishna S. Crosslinking of the electrospun gelatin nanofibers. Polymer. 2006B; 47: 2911-2917.

Zhao M, Song C, Zhang W, Hou Y, Huang R, Song Y, Xie W, Shi Y, Song C. The threedimensional nanofiber scaffold culture condition improves viability and function of islets. $\mathrm{J}$ Biomed Mat Res: Part A. 2009; 94(3): 667-672.

Zhou Q, Brown J, Kanarek A, Rajagopal J, Douglas A. Melton DA. In vivo reprogramming of adult pancreatic exocrine cells to $\beta$-cells. Nature. 2008; 454 (7209): 627-632.

Zimmermann H, Zimmermann D, Reuss R, Feilen PJ, Manz B, Katsen A, Weber M, Ihmig FR, Ehrhart F, Geßner P, Behringer M, et al. Towards a medically approved technology for alginate based microcapsules allowing long term immunoisolated transplantation. J. Materials Sci. 2005; 16: 491-501. 


\section{Appendix A \\ PicoGreen dsDNA Quantification}

An attempt was made to quantify total DNA content by a modified PicoGreen assay (Invitrogen).

Following each analysis by MTS metabolic assay and the final day 21 glucose tolerance test, $10 \%$

DMSO was added to each well, and the plates were frozen at $-80^{\circ} \mathrm{C}$. For analysis, the plates were thawed, the contents transferred to eppendorf vials, and the storage medium was removed by aspiration and washing and replaced with $200 \mu \mathrm{L}$ TE buffer supplemented with 9 mM EDTA and readjusted to $\mathrm{pH}$ 7.4. The DMSO was added as a chelating agent in order to dissolve the bariumalginate particle for full liberation of the DNA. The samples were subjected to five freeze-thaw cycles at $-80^{\circ} \mathrm{C}$ for 30 minutes and $37^{\circ} \mathrm{C}$ for 15 minutes respectively with a quick vortex following each thaw cycle. After particle and cell dissociation by freeze-thaw cycling, $100 \mu \mathrm{L}$ of each sample was placed in a 96-well plate to which $100 \mu \mathrm{L}$ of PicoGreen working reagent was added. A calibration ladder of 0.01-2 $\mu \mathrm{g} / \mathrm{L}$ lambda DNA standard was also prepared and analyzed in the same manner. The plate was allowed to incubate for 3 minutes and was read by spectrophotometer with an excitation of $490 \mathrm{~nm}$ and emission at $520 \mathrm{~nm}$.

The PicoGreen double stranded DNA assay (Invitrogen) was modified to increase the EDTA concentration of the buffer from $1 \mathrm{mM}$ to $10 \mathrm{mM}$. This was performed in order to assist in the disruption of the barium alginate particles during the freeze-thaw cycles required to disrupt the cell membranes and free the DNA. The additional EDTA chelates the barium, dissolving the ionic gel. 
Using only the TE buffer supplied in the assay, five freeze-thaw cycles were insufficient to mechanically disrupt the particles. Through an optimization, it was discovered that $10 \mathrm{mM}$ EDTA could disrupt the particles following three freeze-thaw cycles with a quick vortex after each thaw. The addition of $9 \mathrm{mM}$ EDTA reduced the $\mathrm{pH}$ from 7.4 to 3.18 . The $\mathrm{pH}$ was restored to 7.4 with $1.0 \mathrm{M} \mathrm{NaOH}$.

A test calibration as performed for the $1 \mathrm{mM}$ and $10 \mathrm{mM}$ EDTA systems, with a slight decline in slope and no change in linearity (Figure 47). Therefore, it was decided to use the $10 \mathrm{mM}$ system with a calibration curve prepared under the same conditions.

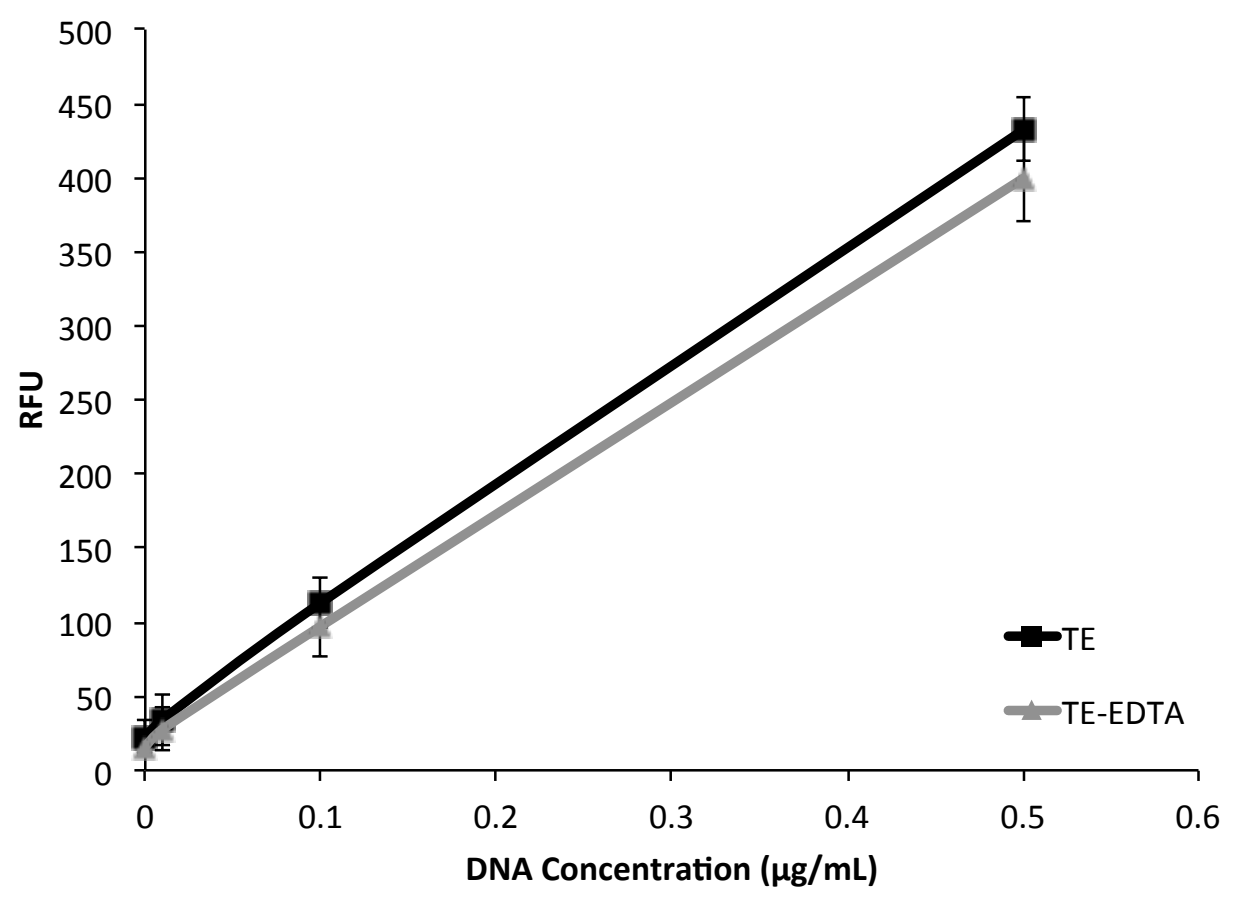

Figure 47. Calibration curve for the standard and $10 \mathrm{mM}$ EDTA PicoGreen assays. (Mean \pm $\mathrm{SD}, \mathbf{n}=3$ ) 


\section{DNA Quantification Results}

The concentration of dsDNA measured was significantly lower than expected. According to Colella et al. (1985), the DNA content of a single islet ranges from 60-210 ng. The samples from the 50-capsule glucose tolerance test were analyzed following day 21 . The dsDNA content for each sample is illustrated in Figure 48, with no significant differences between the sample groups, and an average concentration of only $6.3 \pm 0.3 \mathrm{ng} / \mathrm{well}(\mathrm{n}=23)$. The dsDNA content of the metabolic assay wells collected at each time point for 50 capsules was also only $6.2 \pm 0.9 \mathrm{ng} / \mathrm{well}$ $(\mathrm{n}=24)$.

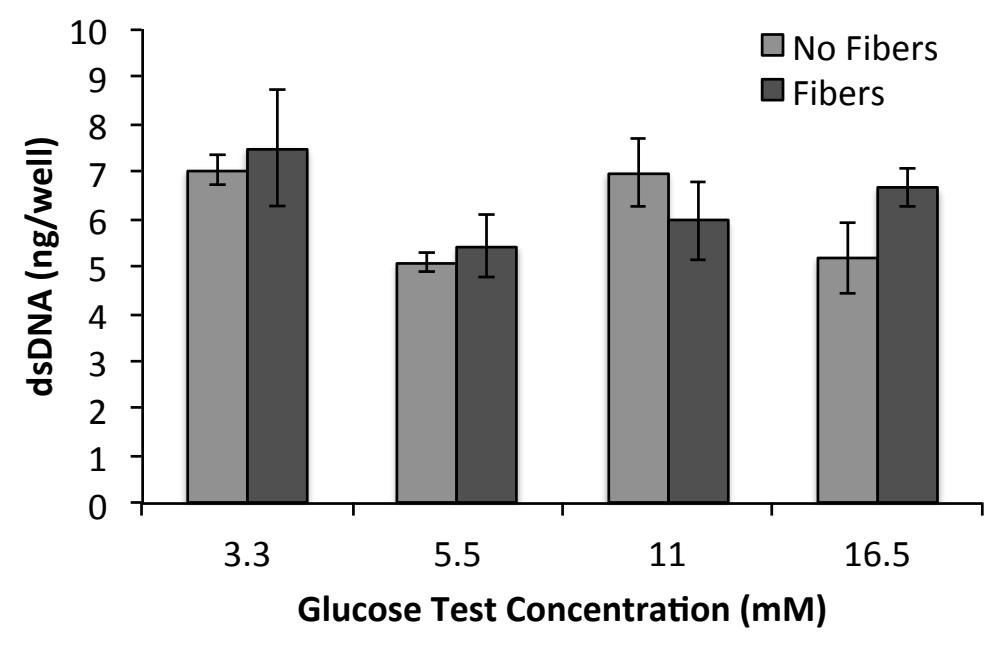

Figure 48. Double stranded DNA content of the glucose tolerance test samples at day 21 as measured by PicoGreen assay. (Mean $\pm \mathrm{SE}, \mathrm{n}=3$ ).

The low dsDNA concentration may be due to DNA diffusion upon washing the particles following thawing from cryostorage in $10 \%$ DMSO, or incomplete cellular lysis due to residual DMSO levels. For this reason, the data was removed from the bulk of the report, but included here for future reference. 


\section{Appendix B}

\section{Preliminary Protein Membrane Investigation}

A preliminary investigation was conducted into the production of crosslinked gelatin membranes on alginate microparticles under cytocompatible conditions. The goal was to develop an ECM membrane to improve the adhesion and spreading of vascular endothelial cells on the surface of the microparticles, emulating earlier modular tissue engineering approaches to vascularization by McGuigan and Sefton (2007). However, the trajectory of the research moved from membranes to matrices and this field of research never extended beyond a preliminary investigation.

Gelatin membranes were formed on the surface of $2 \%$ alginate particles produced by air-assisted dropwise external gelation in $100 \mathrm{mM} \mathrm{CaCl}_{2}$. Alginate microparticles were placed within a $0.5 \%$ solution of gelatin buffered to $\mathrm{pH} 6.0$ with $50 \mathrm{mM}$ MES and incubated for 10 minutes at $38^{\circ} \mathrm{C}$. Following incubation, an aliquot of 5\% Activa microbial transglutaminase was added for a final concentration of $1 \%$ and the mixture was incubated for an additional 10 minutes at $38^{\circ} \mathrm{C}$. The particles were stained with $0.1 \%$ coomassie blue to visualize protein and subjected to dissolution in a $50 \mathrm{mM} \mathrm{Na}_{2} \mathrm{CO}_{3}$ and $20 \mathrm{mM}$ citric acid chelating buffer (Figure 49). 


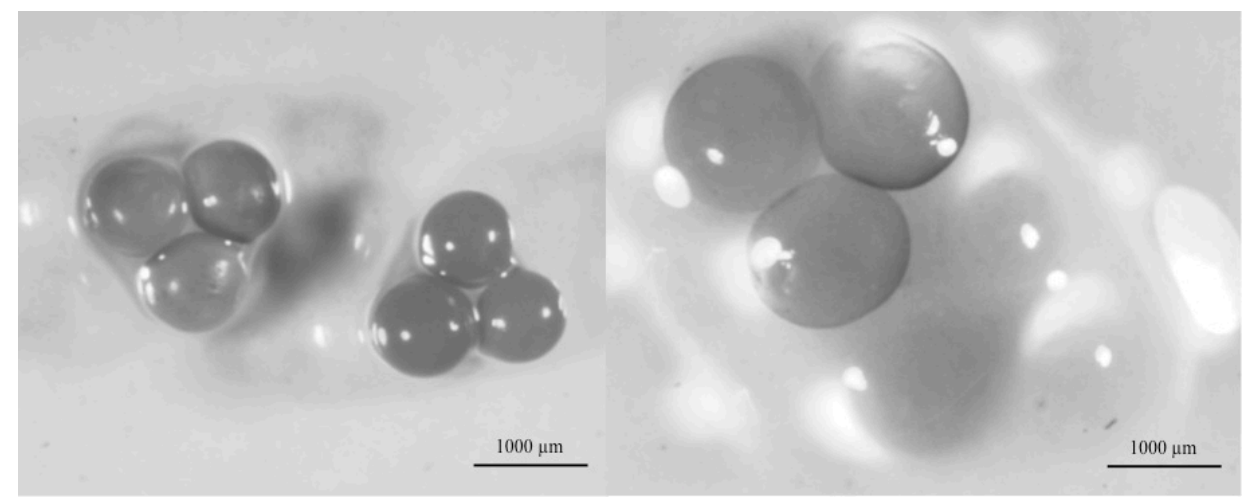

Figure 49. Swelling of alginate particles with and without transglutaminase-crosslinked gelatin membranes. Left: particles with crosslinked gelatin membranes upper left, particles without membranes bottom right, before chelation; Right: after $40 \mathrm{~min}$ in chelating buffer.

The membranes exhibited considerable flexibility and strength, but ultimately failed due to the buildup of osmotic pressure within the particle when incubated in chelating buffer due to the dissolution of the alginate core (Figure 50).

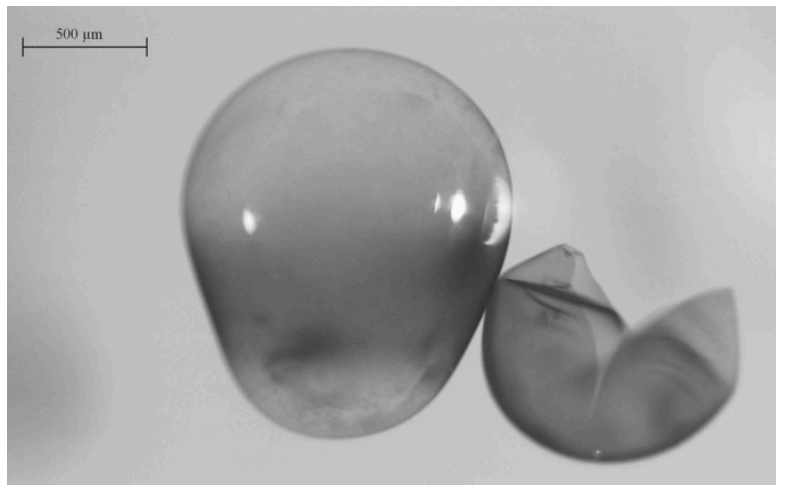

Figure 50. Swollen and ruptured transglutaminase-crosslinked gelatin membranes.

Confocal microscopy was performed on a sample of particles produced from FITC-labeled gelatin (green) and RBITC-labeled alginate (red) (Figure 51). Within the cross-sectional image obtained by confocal microscopy, the alginate core and gelatin membrane are clearly visible, with separation due to the initial stages of alginate dissolution. 


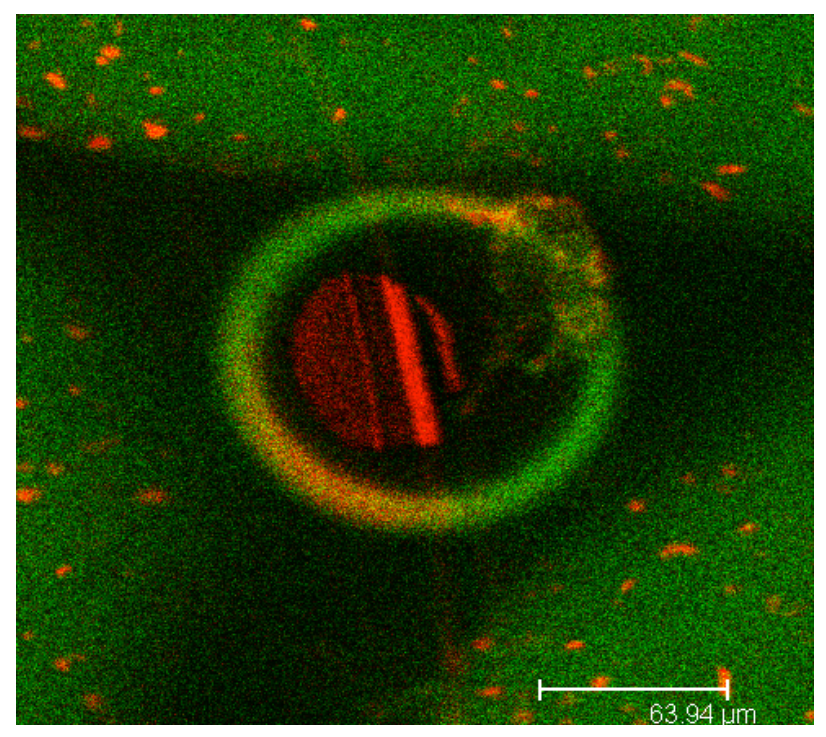

Figure 51. Confocal microscopy image of a FITC-gelatin (green) membrane around a RBITC-alginate (red) core. 